\title{
Accurate unfolding of photon spectra of clinical linear accelerators using transmission measurements
}

by

Elsayed S. M. Ali

A thesis submitted to the Faculty of Graduate and Postdoctoral Affairs

in partial fulfillment of the requirements for the degree of

Doctor of Philosophy

in

Physics

Specialization in Medical Physics

Ottawa-Carleton Institute for Physics

Department of Physics

Carleton University

Ottawa, Ontario, Canada

September 2012

(C) 2012 Elsayed S. M. Ali 
Library and Archives

Canada

Published Heritage

Branch

395 Wellington Street

Ottawa ON K1A ON4

Canada
Bibliothèque et

Archives Canada

Direction du

Patrimoine de l'édition

395 , rue Wellington

Ottawa ON K1A ON4

Canada
Your file Votre référence

ISBN: 978-0-494-93667-2

Our file Notre référence

ISBN: $978-0-494-93667-2$
NOTICE:

The author has granted a nonexclusive license allowing Library and Archives Canada to reproduce, publish, archive, preserve, conserve, communicate to the public by telecommunication or on the Internet, loan, distrbute and sell theses worldwide, for commercial or noncommercial purposes, in microform, paper, electronic and/or any other formats.

The author retains copyright ownership and moral rights in this thesis. Neither the thesis nor substantial extracts from it may be printed or otherwise reproduced without the author's permission.
AVIS:

L'auteur a accordé une licence non exclusive permettant à la Bibliothèque et Archives Canada de reproduire, publier, archiver, sauvegarder, conserver, transmettre au public par télécommunication ou par l'Internet, prêter, distribuer et vendre des thèses partout dans le monde, à des fins commerciales ou autres, sur support microforme, papier, électronique et/ou autres formats.

L'auteur conserve la propriété du droit d'auteur et des droits moraux qui protege cette thèse. $\mathrm{Ni}$ la thèse ni des extraits substantiels de celle-ci ne doivent être imprimés ou autrement reproduits sans son autorisation.
In compliance with the Canadian Privacy Act some supporting forms may have been removed from this thesis.

While these forms may be included in the document page count, their removal does not represent any loss of content from the thesis.
Conformément à la loi canadienne sur la protection de la vie privée, quelques formulaires secondaires ont été enlevés de cette thèse.

Bien que ces formulaires aient inclus dans la pagination, il n'y aura aucun contenu manquant. 


\section{Abstract}

Most cancer radiation therapy treatments use external photon beams from clinical linear accelerators. For these beams, transmission analysis is a potentially viable approach for independent determination of the photon spectra and the incident electron energies. This study presents a comprehensive physics-based approach to transmission analysis to address the computational and experimental limitations of previous studies. On the computational side, energy differentiation is significantly improved by using transmission data from an optimum combination of multiple attenuators and detectors. Detector energy response, photonuclear attenuation, and corrections for non-ideal exponential attenuation are accounted for and found to have a major effect on the unfolding accuracy. For robust unfolding, the spectra are specified using a new, validated functional form with four free parameters, one of which is the incident electron energy. On the experimental side, the validation is performed on a research linac whose photon spectra and electron beam parameters are directly and independently known. The validation includes eight beams from 10 to $30 \mathrm{MV}$, with thick bremsstrahlung targets of $\mathrm{Be}, \mathrm{Al}$, and $\mathrm{Pb}$. The approach is demonstrated on a clinical linac for 6, 10 and $25 \mathrm{MV}$ beams. A protocol is developed to account for many experimental influence quantities, allowing for measurement accuracy of $0.4 \%$ on the smallest signals. The unfolded spectra agree with the benchmark spectra with root-mean-square energy fluence deviations of $4.5 \%$. The accuracy of unfolding the incident electron energy is shown to be $3 \%$. The overall accuracy improvement over the best previous studies is at least a factor of 3 . Photon cross section uncertainties are the ultimate limiting factor of the technique. An upper bound estimate at the $95 \%$ confidence level for these uncertainties is found to be $0.7 \%$, which is more realistic than the currently used 'envelope of uncertainty' of $1-2 \%$. By-products of this study include benchmarking the EGSnrc Monte Carlo system for relative ion chamber response calculations at the $0.2 \%$ level, and upgrading EGSnrc to model photonuclear attenuation. 


\section{Acknowledgments}

I am deeply grateful to my supervisor, Dr. Dave Rogers, for always being there when I turned to him for direction, knowledge and inspiration. I pride myself that I am the only student who was lucky enough to be supervised by Dave in two degrees. My collaborator in the experimental work, Dr. Malcolm McEwen, taught me priceless experimental skills and rigor. His cheerful attitude and insightful discussions made our long experimental days very enjoyable. The members of my advisory committee, Dr. Paul Johns and Dr. James Harden, gave me valuable guidance. Dr. Carl Ross of NRC provided invaluable assistance with the research linac operation and useful discussions of the experimental details.

I greatly appreciate the help of many colleagues in this work. From Carleton University: Bryan Muir, Ben Spencer, Matt Bowcock, Philippe Gravelle, Dr. Tong Xu, Justin Sutherland, Bill Jack and Wade Hong. From NRC: David Marchington, Dr. Frederic Tessier and Dr. Ernesto Mainegra-Hing. From The Ottawa Hospital Cancer Centre: Dr. Dan La Russa, Dr. Jason Belec and Randy Taylor. Discussions with Dr. Steve Seltzer of NIST and Dr. Iwan Kawrakow of Siemens were very insightful.

I'm grateful to the generous support from the following scholarship funding: Vanier, Holbein, Lewar, the Kiwanis Club of Ottawa Medical Foundation and Dr. Kanta Marwah, the Dean of Graduate Studies, and the Physics Department.

To my wife and best friend, Azza: thank you for all your love and support during this demanding period. To my 23-month-old daughter, Leena: I would be remiss if I don't acknowledge your cute contribution of doodling ducks and fishes all over my manuscripts! To my parents: no words can thank you enough for your unconditional love and support. I owe any accomplishment in my life to you.

Dedication: This thesis is dedicated to my mother. 


\section{Statement of originality}

This thesis is a summary of the most significant portion of the author's work during the course of his Ph.D. program at Carleton University. It is based on the journal papers, technical reports and conference abstracts listed below. These publications are referenced throughout the thesis using their Roman letter indices below.

Dr. Dave Rogers supervised the project and provided input on all of its components, including the publications. Dr. Malcolm McEwen supervised the experimental aspects and provided input on the publications. Matt Bowcock and machine shops helped with the design and manufacturing of the experimental components. Ben Spencer compiled the literature cross section data in $\S 6.3$. Otherwise, the author of this thesis designed and built the experimental setup, purchased the different components using Dr. Dave Rogers' research funds, performed the experimental and all the computational work, and drafted and revised all the manuscripts.

\section{Peer-reviewed papers}

I. E. S. M. Ali and D. W. O. Rogers, "Functional forms for photon spectra of clinical linacs", Phys. Med. Biol. 57, $31-50$ (2012).

II. E. S. M. Ali and D. W. O. Rogers, "An improved physics-based approach for unfolding megavoltage bremsstrahlung spectra using transmission analysis", Med. Phys. $39,1663-1675$ (2012).

III. E. S. M. Ali, M. R. McEwen and D. W. O. Rogers, "Detailed high-accuracy megavoltage transmission measurements: A sensitive experimental benchmark of EGSnrc", Med. Phys. 39, 5990 - 6003 (2012).

IV. E. S. M. Ali, M. R. McEwen and D. W. O. Rogers, "Unfolding linac photon spectra and incident electron energies from experimental transmission data, with direct independent validation", Med. Phys., in press (2012).

V. E. S. M. Ali, B. Spencer, M. R. McEwen and D. W. O. Rogers, "Towards a more realistic estimate of the uncertainty of photon cross sections at radiation therapy energies", Phys. Med. Biol., to be submitted (2012). 


\section{Technical reports}

I. E. S. M. Ali and D. W. O. Rogers, "Validation of a new spectral functional form", Technical Report CLRP $11-01$ (15 pp), Carleton University, Ottawa, Canada. http://www.physics.carleton.ca/clrp/brem_spectra, 2011.

II. E. S. M. Ali and D. W. O. Rogers, "Implementation of photonuclear attenuation in EGSnrc", Technical Report CLRP 12 - 01 (14 pp), Carleton University, Ottawa, Canada. http://www.physics.carleton.ca/clrp/photonuclear, 2012.

III. E. S. M. Ali, M. R. McEwen and D. W. O. Rogers, "Data for an accurate transmission measurement benchmark", Technical Report CLRP $12-02$ (15 pp), Carleton University, Ottawa, Canada. http://www.physics.carleton.ca/clrp/transmission, 2012.

Conference abstracts (all are oral presentations; * indicates presenting author)

I. E. S. M. Ali*, M. R. McEwen and D. W. O. Rogers, "Computational and experimental methods to address the limitations of reconstructing linac photon spectra from transmission measurements", Proc. 56th COMP Annual Scientific Meeting, Ottawa, Ontario, Canada, Jun. 2010, p 9. Also published in Med. Phys. 37, 3884 (2010). Finalist, J. R. Cunningham Young Investigators Symposium.

II. E. S. M. Ali and D. W. O. Rogers*, "Functional forms for photon spectra of clinical linacs", 11th biennial ESTRO meeting on Physics and Radiation Technology for Clinical Radiotherapy, London, England, May 2011. Published in Radiother. Oncol. 99 suppl. 1, 378 (2011).

III. E. S. M. Ali", M. R. McEwen and D. W. O. Rogers, "Monte Carlo methods for the inverse problem of spectral unfolding", Proc. 4th McGill Int'l Conf. on Recent Advances in Monte Carlo Techniques for Radiation Therapy, Montréal, Canada, Jun. 2011, p 37.

IV. E. S. M. Ali", M. R. McEwen and D. W. O. Rogers, "Beyond self-consistency in beam commissioning: Determination of true linac spectra", Joint AAPM (53rd)/ COMP (57th) Annual Scientific Meeting, Vancouver, Canada, Jul./Aug. 2011. Published in Med. Phys. $383870-3871$ (2011).

V. E. S. M. Ali", M. R. McEwen and D. W. O. Rogers, "A validated approach for clinical linacs to accurately determine the photon spectra and the incident electron energy", Proc. 58th COMP Annual Scientific Meeting, Halifax, Nova Scotia, Canada, Jul. 2012, p 15. Winner of 2nd place, J. R. Cunningham Young Investigators Symposium. 


\section{Contents}

1 Introduction 1

1.1 The importance of accurate photon spectra $\ldots \ldots \ldots \ldots$

1.2 Methods to determine photon spectra . . . . . . . . . . . 2

1.3 Bremsstrahlung .......................... 4

1.4 Ion chambers in photon beams $\ldots \ldots \ldots \ldots$

1.5 The EGSnrc Monte Carlo system . . . . . . . . . . . . . . 6

1.6 Transmission analysis $\ldots \ldots \ldots \ldots$

1.7 Limitations of previous studies $\ldots \ldots \ldots \ldots$

1.8 Motivation, goals, and thesis organization . . . . . . . . . 12

2 The physics-based approach $\quad 14$

2.1 New functional form .................... 14

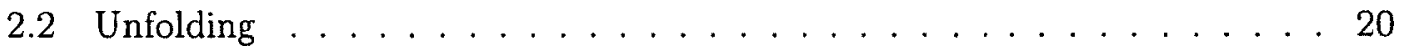

2.3 Accurate detector energy response modelling f . . . . . . . . . . 21

2.4 Improving energy differentiation $\ldots \ldots \ldots \ldots \ldots$

2.5 Optimizing the measurement configuration . . . . . . . . . 26

2.6 Photonuclear attenuation $\ldots \ldots \ldots \ldots \ldots$

2.7 Correction for non-ideal attenuation . . . . . . . . . . . 34

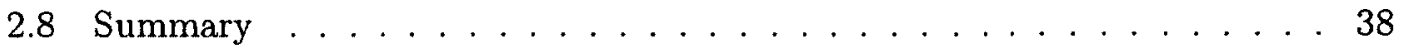


3.1 The NRC research linac $\ldots \ldots \ldots$. . . . . . . . . . . . . . 39

3.2 Transmission measurement setup . . . . . . . . . . . . . . . 42

3.3 Data acquisition and experimental corrections . . . . . . . . . . 48

3.4 Experimental uncertainty budget . . . . . . . . . . . 54

3.5 Clinical linac measurements . . . . . . . . . . . . . . . 58

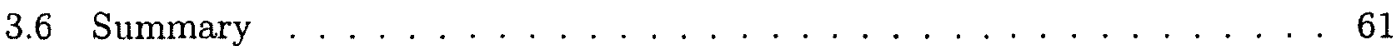

4 A sensitive EGSnrc benchmark $\quad 62$

4.1 Modelling the research linac setup . . . . . . . . . . . . . . 62

4.2 Bremsstrahlung angular sampling . . . . . . . . . . . . 63

4.3 Modelling photonuclear attenuation . . . . . . . . . . 64

4.4 Monte Carlo uncertainty budget . . . . . . . . . . . . . 67

4.5 Sensitivity of transmission data (relative comparisons) . . . . . . . . 68

4.6 Direct comparisons of transmission data . . . . . . . . . . . . . 71

4.7 Summary . . . . . . . . . . . . . . . . . . 74

5 Unfolded spectra and incident electron energies $\quad 75$

5.1 Research linac direct independent validation methods . . . . . . . . . 75

5.2 Clinical linac validation methods . . . . . . . . . . . . 78

5.3 Unfolding using experimental data . . . . . . . . . . . . 79

5.4 Unfolded data for the research linac . . . . . . . . . . . 81

5.5 Unfolded data for the clinical linac . . . . . . . . . . . . 87

5.6 Higher transmission cutoffs . . . . . . . . . . . . . . 91

5.7 Summary . . . . . . . . . . . . . . . . . . 91 
6 Estimating photon cross section uncertainties

6.1 Method of analysis ... . . . . . . . . . . . . . 93

6.2 Estimation using transmission data . . . . . . . . . . . . . 94

6.3 Estimation using literature cross section measurements . . . . . . . . . 97

6.4 Discussion . . . . . . . . . . . . . . . . . . . 99

6.5 Summary . . . . . . . . . . . . . . . . . . 101

$\begin{array}{lll}7 & \text { Summary and conclusions } & 102\end{array}$

7.1 Summary of how previous limitations were addressed . . . . . . . . 102

7.2 Recommendations for accurate transmission rneasurements . . . . . . . 104

7.3 Conclusions . . . . . . . . . . . . . . . . . 106

7.4 Future work . . . . . . . . . . . . . . . . . 108

7.5 Potential applications f. . . . . . . . . . . . . 109

$\begin{array}{ll}\text { Appendices } & 111\end{array}$

$\begin{array}{ll}\text { A Benchmark set of spectra } & 111\end{array}$

B Previous functional forms $\quad 113$

C Refining NIST XCOM photon cross sections in EGSnrc 121

D Implementing photonuclear attenuation in EGSnrc 125

$\begin{array}{ll}\text { References } & 127\end{array}$ 


\section{List of Tables}

2.1 Performance of different attenuator/detector configurations . . . . . . . . 29

3.1 Bremsstrahlung target dimensions for the research linac . . . . . . . . 41

3.2 Experimental uncertainty budget for the research linac measurements . . 55

4.1 Monte Carlo uncertainty budget . . . . . . . . . . . . . 68

5.1 Differences between transmission and NaI measurement setups . . . . . . 77

5.2 Accuracy of the unfolded data for the research linac . . . . . . . 84

5.3 Unfolded data for the clinical linac . . . . . . . . . . . . . 990

6.1 Estimates of photon cross section uncertainties . . . . . . . . . . . 97

B.1 Standardized notation to describe and evaluate functional forms . . . . . 114

B.2 Equations of different functional forms . . . . . . . . 115

B.3 Accuracy and robustness of different functional forms . . . . . . . 118 


\section{List of Figures}

2.1 Examples of spectral fits using the proposed functional form . . . . . . . 19

2.2 Detector response modelling: Configuration and results . . . . . . . . . . 22

2.3 Effect of detector energy response on spectral unfolding accuracy . . . . . 23

2.4 Rate of change of the mass attenuation coefficient with energy . . . . 26

2.5 Objective function for different measurement configurations . . . . . . 30

2.6 Spectral confidence bounds for different measurement configurations . . . 31

2.7 Fractional photonuclear cross sections for the attenuator materials . . . . 32

2.8 Effect of photonuclear attenuation on transmission . . . . . . . . . 33

2.9 Effect of photonuclear attenuation on spectral unfolding accuracy . . . 33

2.10 Schematic setup for studying non-ideal attenuation conditions . . . . . 34

2.11 Spectra of non-primary photons at the detector location . . . . . . 36

2.12 Effect of non-ideal conditions on transmission data . . . . . . . . . 36

2.13 Effect of non-ideal conditions on spectral unfolding accuracy . . . . . . 37

3.1 The NRC Vickers research linac . . . . . . . . . . . . . . 40

3.2 Components of the measurement setup on the research linac . . . . . 46

3.3 A view of the full measurement setup on the research linac . . . . . 47

3.4 Horizontal profiles for the research linac beams . . . . . . . . . . . 47

3.5 Monitoring beam drifts in the research linac . . . . . . . . . . 50

3.6 Polarity correction for the research linac measurements . . . . . . . . 52 
3.7 A view of the full measurement setup on the clinical linac . . . . . . 60

3.8 Polarity correction for the clinical linac measurements . . . . . . . . . 60

4.1 Bremsstrahlung angular sampling for the research beams . . . . . . 65

4.2 Fractional photonuclear cross sections for the research linac targets . . . 65

4.3 Photonuclear effect: calculated versus measured transmission . . . . . . 66

4.4 Measured and calculated sensitivities of transmission data . . . . . . 69

4.5 Example of measured and calculated transmission curves . . . . . . . . 72

4.6 Ratio of measured-to-calculated transmission for the research beams . . . 73

5.1 Transfer factor from transmission setup to NaI measurement setup . . . . 77

5.2 Correction factors for non-ideal attenuation conditions . . . . . . . 80

5.3 Unfolded spectra of the research linac beams . . . . . . . . . . . 82

5.4 Examples of some effects on the accuracy of the unfolded spectra . . . . 86

5.5 Measured and calculated transmission data for the clinical beams . . . 88

5.6 Unfolded spectra of the clinical linac beams . . . . . . . . . . . . 89

6.1 C photon cross section scaling for transmission analysis . . . . . . . 96

$6.2 \mathrm{~Pb}$ photon cross section scaling for transmission analysis . . . . . . . 96

6.3 $\mathrm{C}$ and $\mathrm{Pb}$ photon cross section scaling for literature data analysis . . . . 99

6.4 Measured literature cross sections versus XCOM + IAEA photonuclear . 100

B.1 Examples of spectral fits using functional forms from the literature . . 120

C.1 Incoherent scattering cross section: EGSnrc versus XCOM . . . . . . 122

C.2 Pair + triplet cross section: EGSnrc versus XCOM . . . . . . . . . . 123

C.3 Total photon cross section: EGSnrc versus.XCOM . . . . . . . . . . . 124

D.1 Validation of photonuclear implementation in EGSnrc . . . . . . . . 127 


\section{Chapter 1}

\section{Introduction}

In cancer therapy, about $30-50 \%$ of the patients receive some form of radiation treatment, and about $90 \%$ of these treatments use external photon beams from clinical linear accelerators (linacs). For modern linacs in photon mode, electrons are accelerated through a wave guide to energies typically within $4-20 \mathrm{MeV}$. The electrons are guided and focused using bending magnets until they reach the photon target, which is typically made of a high-Z material thick enough to stop the electrons. The incident electrons decelerate in the target due to Coulomb interactions, leading to radiative losses known as bremsstrahlung (German for 'breaking radiation'). The energy distri-

bution of the resulting photon beam is altered by the target self-attenuation and by modifiers that may exist in the beam path (flattening filters, wedges, etc). A clinical photon beam is given a nominal MV value, which is not necessarily equal to the incident electron beam energy. The common beams are 6, 10, 15, 18, and 25 MV. An important part of beam characterization is the specification of its photon spectrum.

\subsection{The importance of accurate photon spectra}

The photon spectrum is one of the inputs to the dose calculation engines in treatment planning systems (TPSs). The current approach in commissioning photon beams is to tune the photon spectrum in the TPS to match the measured commissioning data 
(typically depth-dose curves and beam profiles). This 'self-tuning' approach can mask issues in the linac and in the TPS. It also does not guarantee that the beam model is correct for non-standard treatment conditions - e.g., for small fields, and around tissue interfaces and heterogeneities. ${ }^{1,2}$ As TPSs move towards Monte Carlo engines and more detailed patient and beam modeling, the accuracy of the input spectrum is becoming more important. Therefore a reliable independent method to determine photon spectra would be useful for more robust beam commissioning/re-commissioning, and for stricter testing of TPSs. Spectral accuracy is also important for modelling the energy response of detectors in a given beam, ${ }^{3}$ and for calculations of spectrum-averaged dosimetric quantities and detector correction factors, particularly for the development of dosimetry protocols. ${ }^{4,5}$ For cases where accurate spectral measurements are performed on a linac whose geometry and incident electron parameters are independently known (e.g., the research linac used in this study), the measured spectra are useful as a primary benchmark for Monte Carlo codes. ${ }^{6}$

\subsection{Methods to determine photon spectra}

Methods to determine photon spectra can be classified as semi-analytical, Monte Carlo, spectroscopy or indirect measurements. These methods are briefly highlighted here.

Semi-analytical spectral calculations combine bremsstrahlung theories with reasonable approximations to account for the spreading and slowing down of electrons in a thick target, ${ }^{7,8}$ and they could also include the first scatter of photons. ${ }^{9}$ Such methods are not suitable today given the sophistication of modern linacs and treatment planning.

Monte Carlo methods include detailed radiation transport, and can model complex situations. However, they are fundamentally limited by their underlying physics mod-

els and cross section data. Also, they can never replace measurements since physical changes cannot be detected (e.g., misalignment of a flattening filter).

\subsection{METHODS TO DETERMINE PHOTON SPECTRA}


Spectroscopy methods include direct and Compton spectroscopy. Direct spectroscopy has been performed in a hospital setting using a typical clinical linac, ${ }^{10}$ and in a research environment using a special linac. ${ }^{6,11,12}$ However, such measurements require ultra-low dose rates to avoid detector saturation. This requirement may not be available for many clinical linacs. If available, it would require unusual manipulation of fundamental linac operating components, which may lead to differences between the measured and the therapeutic spectra due to differences in the electron beam parameters. The setup is also complex, expensive and requires excessive shielding and long linac down time. In Compton spectroscopy, the dose rate is reduced by placing a scatterer at an angle in the photon beam and measuring the energy distribution of the Compton-scattered photons or electrons. ${ }^{13-15}$ The incident photon spectrum is extracted from the known kinematics of Compton scattering. Except for the reduced dose rate, this method has the same challenges of direct spectroscopy. In addition, there is a loss of resolution because the energy range of the Compton-scattered spectrum is more compact than that for the incident spectrum. There is also reduced sensitivity at higher energies because of the lower Compton cross section and the weaker dependence of the scattering angle on energy.

Indirect measurements involve unfolding the spectrum from measurements of another physical quantity. Many indirect methods have interesting underlying physics principles; however, they suffer from major limitations that make them not useful clinically. Examples of such interesting but impractical methods include: (a) irradiating a material in the photon beam and counting the produced electron-positron pair tracks using a cloud chamber or a magnetic field ${ }^{16,17}$ (b) photo-disintegration of deuterium then measuring the energy of the resulting protons and/or neutrons; ${ }^{18}$ and, (c) photoactivation of the nuclei of a series of foils for different elements then measuring the resulting activity. ${ }^{19}$ The only two indirect methods which are clinically viable are trans-

\subsection{METHODS TO DETERMINE PHOTON SPECTRA}


mission measurements and depth-dose measurements. In transmission analysis, which is the topic of this study, the spectrum is unfolded from transmission signals acquired in narrow-beam geometry after the beam passes through successive thicknesses of an attenuating material. Depth-dose analysis is similar to transmission, except that the signals are acquired at multiple depths in a water tank (phantom) ${ }^{20-22}$ Depth-dose measurements are routinely done in the clinic; however, the unfolding is more difficult than it is for transmission because of the reduced energy differentiation, the electron contamination, the field-size dependence, and the phantom scatter. A parallel study on depth-dose analysis has been performed, and it will be presented elsewhere. ${ }^{23}$

\subsection{Bremsstrahlung}

Much of the bremsstrahlung production occurs in the first 'thin' layer of the target because the incident electron energy degrades quickly in the high-Z material and the bremsstrahlung cross section reduces almost linearly with this energy degradation. The mean photon emission angle (in radians) is equal to the ratio of the electron rest mass to its total energy. The combined effect of the two statements above is that bremsstrahlung

production is strongly forward peaked at megavoltage energies. Thin-target formulae for electron-nuclear bremsstrahlung production are summarized in the definitive review article of Koch and Motz. ${ }^{24}$ The following features of those formulae are highlighted because of their relevance to this study. (a) The relatively simpler formulae (e.g., the Schiff spectrum ${ }^{8,25}$ ) make use of the Born approximation which assumes a free electron wave function that interacts with the nuclear Coulomb potential via the production of only one bremsstrahlung photon per interaction. The Born approximation is less accurate for high- $Z$ targets, for low initial electron energies, and for high-energy bremsstrahlung photons (called the high-frequency limit). Corrections to the Born approximation are called Coulomb corrections, and they have only been determined at

\subsection{BREMSSTRAHLUNG}


extreme relativistic energies (above $50 \mathrm{MeV}$ ). (b) Some formulae include screening corrections to account for the screening of the nucleus by the orbital electrons, which is more important for high- $\mathrm{Z}$ targets and for low electron energies. (c) The contribution of electron-electron bremsstrahlung can be approximated by replacing the square dependence on the atomic number of the target material, $Z^{2}$, with $Z(Z+1)$. (d) All formulae do not include electron or photon polarization effects.

The National Institute of Standards and Technology (NIST) provides cross section data for bremsstrahlung production, differential in electron energy. The data are based on the compilation of Berger and Seltzer ${ }^{26,27}$ for electrons with kinetic energy from $1 \mathrm{keV}$ to $10 \mathrm{GeV}$ incident on neutral atoms with $\mathrm{Z}=1-100$. The data use Coulomb-corrected extreme relativistic cross sections above $50 \mathrm{MeV}$, partial wave analysis calculations by Tseng and $\operatorname{Pratt}^{28}$ below $2 \mathrm{MeV}$, and cubic-spline interpolations between 2 and $50 \mathrm{MeV}$. The data include the effect of electron-electron bremsstrahlung. The energy range $2-50 \mathrm{MeV}$ has the largest uncertainties, which are estimated to be $10 \%$ to $3 \%$ going from 2 to $50 \mathrm{MeV}$.

\subsection{Ion chambers in photon beams}

A typical ion chamber consists of a gas-filled cavity between two electrodes. Ion chambers are the most widely used detectors in radiation dosimetry, and they are the detectors of choice in this study. Their widespread use is because of their reliability, high-precision, established history, and well-understood correction factors. In photon beams, the most commonly used reference-class chambers are 0.6 cc cylindrical chambers, vented to the surrounding air. Cylindrical chambers with a conical top are typically called 'Farmer chambers', after the original design by Baldwin and Farmer. ${ }^{29}$

In an ion chamber measurement in photon beams, photons interact in the chamber wall and generate secondary electrons that cross the cavity and ionize its air. The col- 
lected ionization signal is proportional to the dose (energy per unit mass) deposited in the cavity. In-air measurements require the use of a 'buildup cap' to generate enough secondary electrons for a reasonable-size signal. 'Full buildup' is achieved when the cap thickness is at least equal to the range of secondary electrons in the cap material. Correction factors are applied to the raw chamber signals to account for certain variations in measurement conditions. ${ }^{4}$ Relevant to this study are the corrections for polarity, $P_{p o l}$, ion recombination, $P_{i o n}$, and temperature-pressure, $P_{T P}$. The $P_{p o l}$ correction accounts for the difference in the signals when the polarities of the electrodes are reversed. The effect is partly related to chamber construction details, and partly to extra-cameral signals in the chamber cables. The $P_{i o n}$ correction accounts for the lack of complete charge collection efficiency due to the recombination of ion pairs. Some of the recombination occurs between pairs from different ionization tracks (called general recombination), therefore $P_{i o n}$ depends on the dose rate and the linac pulse rate. The $P_{T P}$ correction accounts for the fluctuations in the ambient temperature and pressure.

\subsection{The EGSnrc Monte Carlo system}

EGSnrc (Electron Gamma Shower) ${ }^{30,31}$ is a Monte Carlo code system which simulates the coupled transport of electrons and photons in arbitrary geometries in the keV to $\mathrm{GeV}$ energy range. The code has been in development (through its predecessors) for more than three decades, and it is currently considered the gold standard in medical physics. The code was shown ${ }^{32}$ to be accurate within $0.1 \%$ with respect to its own cross sections for relative ion chamber response calculations (called the Fano test).

For charged particles (electron and positron), EGSnrc uses an artifact-free condensed history approach, which increases the simulation efficiency over single scattering calculations by many orders of magnitude. In addition to the basic charged-particle interactions, the code models spin effects, density effects and electron impact ioniza-

\subsection{THE EGSNRC MONTE CARLO SYSTEM}


tion. ${ }^{33}$ For the input electron cross section data, the most accurate option offered in EGSnrc is the NIST compilation discussed earlier in $\S 1.3$, except that the electronelectron bremsstrahlung component is replaced with a more accurate model. ${ }^{34}$ The bremsstrahlung angular sampling in EGSnrc is of particular interest to this study. The two sampling options available are called KM and Simple. The KM option is a modification of the 2BS formula from Koch and Motz. ${ }^{24}$ It offers a compromise between relaxing the extreme-relativistic and the small-angle approximations on one hand, and accounting for the nuclear screening effect on the other hand. The Simple option uses only the leading term of the KM option for faster sampling. Simple is more widely used than KM for simulation efficiency considerations.

For photons, in addition to the basic interactions, the code models orbital electron binding effects, radiative Compton corrections, double Compton scattering, atomic relaxations, and explicit triplet production. The most accurate option offered in EGSnrc for photon cross sections is the NIST XCOM compilation, ${ }^{35}$ except for incoherent scattering cross sections which are calculated internally. As part of this study, the incoherent scattering cross sections from XCOM are made available in EGSnrc; the resolutions of the input pair and triplet cross sections from XCOM are refined in EGSnrc; and, the photonuclear cross sections from the IAEA compilation ${ }^{36}$ are added.

The EGSnrc system offers a series of 'usercodes' tailored to model certain geometries and to score certain quantities. In this study, the usercode BEAMnrc ${ }^{37,38}$ is used to model the linac heads and the transmission measurement setup, while the usercode cavity (which uses the recent egs++ geometry package ${ }^{39}$ ) is used to model the detector details. BEAMnrc is then used as a shared library input to cavity, which eliminates the need for intermediate particle phase-space storage and for particle recycling. The usercode FLURZnrc ${ }^{40}$ is used for calculations of spectra where variable-size energy bins are required. 
The EGSnrc system and its usercodes offer several powerful Variance-Reduction Techniques (VRTs) which significantly improve the simulation efficiency without biasing the results. Names of some of the VRTs, used in this study are directional bremsstrahlung splitting (DBS) ${ }^{41}$ photon splitting and electron range rejection.

\subsection{Transmission analysis}

In transmission analysis, the photon spectrum is unfolded from transmission signals acquired in narrow-beam geometry after the beam passes through different attenuator thicknesses. Historically, the use of transmission analysis to unfold spectra was proposed in the early days of $\mathrm{x}$ rays in the context of kilovoltage applications. ${ }^{42}$ Its application migrated to megavoltage photon beams in the early 1980 s. ${ }^{43}$ The technique was found to be clinically viable because the setup is relatively simple, cost effective and does not require specialized equipment, and because (with due rigor) the measurement accuracy is reasonably achievable in a typical clinical setting.

In an ideal attenuation geometry, the transmission signals can be expressed in terms of the unknown spectrum through a homogeneous Fredholm equation of the first kind given by

$$
T_{\text {ideal }}\left(d, x_{i}\right)=\frac{M\left(d, x_{i}\right)}{M(d, 0)}=\frac{\int_{E_{l}}^{E_{m}} R(d, E) \psi(E) \exp \left[-\frac{\mu}{\rho}(E) x_{i}\right] d E}{\int_{E_{l}}^{E_{m}} R(d, E) \psi(E) d E}
$$

where $M(d, 0)$ and $M\left(d, x_{i}\right)$ are, respectively, the measured signal of detector $d$ (most commonly an ion chamber) without an attenuator and with an attenuator of mass thickness $x_{i}, T_{i d e a l}\left(d, x_{i}\right)$ is the corresponding transmission signal, $R(d, E)$ (called henceforth the energy response of detector $d$ ) is the ion-chamber air-cavity dose per unit energy fluence from monoenergetic photons of energy $E, \psi(E)$ is the unknown photon energy fluence at energy $E$ at the detector location, $\frac{\mu}{\rho}(E)$ is the mass attenuation coefficient 
of the attenuator material at $E$, and $E_{l}$ and $E_{m}$ are the lowest and maximum photon energy, respectively.

The difficulty of the inverse problem of spectral unfolding from measured transmission signals is well known, and it stems from the weak energy dependence of the attenuation coefficient at megavoltage energies. In such an ill-conditioned problem, noise and systematic errors are amplified, and they strongly drive the accuracy of the unfolded data. This increases the requirements on the accuracy and precision of all the components that could lead to noise or to systematic errors. Such components include the measured transmission signals, the numerical methods used for the unfolding, and the components that describe the system kernel in Eq.1.1 - i.e., the detector energy response, the attenuator mass thickness, and the mass attenuation coefficient.

\subsection{Limitations of previous studies}

Previous studies on transmission analysis (listed below) suffer from a number of serious limitations which can be classified under the headings below.

Lack of direct independent validation: The photon spectra of clinical linacs are typically not known independently, therefore most previous experimental studies resorted to indirect validation approaches. In these approaches, comparisons were made between measured spectrum-averaged dosimetric quantities and the calculations of the same quantities using the spectra unfolded from transmission measurements. Such spectrumaveraged quantities include transmission curves, percent depth-dose curves, ${ }^{44-47}$ tissue phantom/maximum ratios, ${ }^{44,48,49}$ stopping power ratios, ${ }^{45,48,50,51}$ mass energy absorption coefficient ratios, ${ }^{45}$ and contrast in portal images. ${ }^{52}$ These indirect validation approaches do not reveal errors in the spectral shapes, and they are not generally sensitive

to slight spectral changes. In a limited number of previous studies, ${ }^{49,53-55}$ the unfolded spectra were visually compared with generic spectra from other studies.

\subsection{LIMITATIONS OF PREVIOUS STUDIES}


Limited energy differentiation: The weak energy dependence of the attenuation coefficient leads to limited energy differentiation in the measured transmission signals. Previous studies did not explore physics methods related to the detectors and/or attenuators to address this fundamental limitation. Rather, most efforts focused on developing unfolding algorithms clever enough to extract useful spectra despite the limited energy differentiation. An exception is the work of Huang et al ${ }^{56}$ in which a combination of $\mathrm{Pb}$ and $\mathrm{Al}$ attenuators is used in one transmission curve to increase energy differentiation. However, their condition for switching the attenuator material makes their technique applicable only to very low-MV beams (e.g., $4 \mathrm{MV}$ ).

Inability to unfold the maximum energy: In previous studies, the robustness of the minimization necessitated that the maximum photon energy, $E_{m}$, be fixed to an assumed value. However, in a typical clinical linac $E_{m}$ is not known, and fixing it to an assumed value, typically the nominal MV of the beam, can be a gross approximation. For instance, for Siemens KD $18 \mathrm{MV}$ and Elekta SL $25 \mathrm{MV}$ beams, the incident electron energy, $E_{e}$, is only 14.7 and $19.0 \mathrm{MeV}$, respectively ${ }^{57}$ (note that $E_{m}=E_{e}$ when the incident electron beam does not have energy spread). Baker and Peck ${ }^{44}$ determined $E_{m}$ by restricting their three-parameter spectral model to only one parameter to unfold $E_{m}$, then fixed it at that value during the full minimization. There is no previous study which treated $E_{e}$ or $E_{m}$ as a truly free parameter.

Systematic errors in the system kernel: One of the main components of the system kernel in the right-hand side of Eq. 1.1 (p.8) is the ion chamber energy response, which was grossly approximated in previous studies. In this study, it is shown that the most commonly used energy response approximation leads to significant errors in the unfolded spectra. To the author's knowledge, there are no previous studies on transmission analysis which used Monte Carlo for detailed detector response modelling. The attenuation coefficient is another important component in the system kernel. The effect of the accu-

\subsection{LIMITATIONS OF PREVIOUS STUDIES}


racy and resolution of the cross section data used, and the contribution of photonuclear interactions to the total attenuation coefficient have been previously ignored. Photonuclear attenuation is shown in this study to have a significant effect on the accuracy of the unfolded high-MV spectra. Finally, the effects of the deviations from ideal narrow-beam geometry on the system kernel have been mostly ignored, particularly those deviations that are difficult to be corrected for experimentally - e.g., forward scatter from the attenuator; attenuation and scatter in the intervening air; and, collimator effects.

Limited robustness: Several methods were previously investigated to improve the minimization robustness. While different methods demonstrated some success under custom conditions, they typically have strong caveats that limit their portability and usefulness. The following is a summary of the limitations of the commonly used methods. Except for the Laplace transform approach, all methods below require discretization of Eq. 1.1 (p.8)'. (a) Laplace methods ${ }^{43,58,59}$ use a 'pair' of functions with a few free parameters to respectively describe the transmission curve and a modified version of the integrand of Eq. 1.1 such that the former is the Laplace transform of the latter. The free parameters are found by directly fitting the transmission curve. Laplace methods are extremely sensitive to the chosen pair and they require the attenuation coefficient to be a strong function of energy. Therefore they are not suitable for typical linac spectra, even for lower-MV beams. ${ }^{60}$ (b) Direct matrix inversion methods ${ }^{51,61}$ are extremely sensitive to the experimental and numerical noise. (c) Neural network methods ${ }^{62}$ are limited by the scope, resolution and accuracy of the training sets. (d) Bin-by-bin iterative unfolding methods $46,48,50,53,54,63,64$ are the most commonly used approach. Different iterative algorithms require different degrees of a-priori spectral knowledge, which may include fixing the maximum or the modal energy. To avoid non-physical spectral shapes in these methods, variations of the spectrum during minimization are controlled by using smoothing constraints and/or regularization. The limitation of these full iterative meth-

\subsection{LIMITATIONS OF PREVIOUS STUDIES}


ods is that the unfolded spectra are sensitive to the initial estimate of the spectrum, the penalty/smoothing function, the regularization parameter, etc. The alternative of not using such constraints leads to non-robust unfolding. (e) Spectrum parameterization methods ${ }^{47,55,65-67}$ specify the spectrum using a functional form with a few free parameters, and the problem reduces to finding those free parameters. This approach has the most potential for true robustness. However, as shown in this study, the functional forms used in previous investigations have many limitations related to their accuracy, flexibility and robustness.

Limited experimental accuracy: In previous experimental studies, there has been only partial $^{44,51,53,63}$ or no effort to investigate the influence quantities that affect the measurement accuracy, and to develop the necessary correction methods and quantify the associated uncertainties. Since the unfolding problem is ill-posed, ignoring the noise and the systematic errors in the measured signals adversely affects the unfolding accuracy. It also does not allow for obtaining meaningful confidence bounds on the extracted spectral information.

Lack of a comprehensive study: In any given previous investigation, attention was focused on certain aspects of the problem, most commonly the unfolding algorithm. The literature is lacking an investigation which addresses the limitations in the physics, numerical and experimental aspects of the problem in a single comprehensive study.

\subsection{Motivation, goals, and thesis organization}

Motivation: This study was motivated by access to two investigation tools. The first is the dedicated research linac at the National Research council (NRC), whose photon spectra were previously measured for different bremsstrahlung targets using a $\mathrm{NaI}$ detector, ${ }^{6,11,12}$ and whose electron beam parameters are accurately and independently known. ${ }^{68,69}$ This allows for the unique ability to perform direct independent valida- 
tion of the unfolded spectra. The second tool is the sophisticated EGSnrc Monte Carlo system which enables in-depth investigations of the different aspects of the problem.

Goals: There are two goals for this study. The first is to develop and validate an approach/tool to accurately determine linac photon spectra and incident electron energies from measured transmission signals. This tool can improve linac beam commissioning and spectral quality assurance, and can be used to provide a library of measured spectra for the currently available linac photon beams. The second goal is to provide comprehensive work on the different aspects of transmission analysis at megavoltage energies in a single study. To achieve these two goals, new and improved methods are developed to address the limitations of previous studies. The proposed approach is validated on the NRC research linac and demonstrated on a typical clinical linac. By-products of this work include upgrading and validating the EGSnrc system, providing accurate transmission data to serve as a sensitive experimental benchmark for other Monte Carlo codes, and, estimating the uncertainty of photon cross sections at megavoltage energies.

Thesis organization: This thesis is organized as follows. In Ch. 2, the theoretical aspects of an improved physics-based approach to unfold spectra using transmission analysis are presented, along with their computational validation. In Ch. 3, the experimental details are discussed for the research and the clinical linacs. In Ch. 4, the experiment is modelled using Monte Carlo, and the results are used for benchmarking EGSnrc and for sensitivity studies. In Ch. 5 , the experimental data are used with the proposed approach to unfold spectral data, and the results of the validation are presented. In Ch. 6 , photon cross section uncertainties are estimated using two different experimental data sets. For better information flow throughout this thesis, intermediate results are presented in the same sections where their respective methods are developed. App. A and B are referred to in Ch. 2; App. C is referred to in Chs. 2 and 4; and, App. D is referred to in Ch. 4. 


\section{Chapter 2}

\section{The physics-based approach}

This chapter starts by introducing and validating a new functional form for linac photon spectra $(\S 2.1)$, which is used during spectral unfolding. The unfolding details are presented upfront $(\$ 2.2)$ because they apply to all subsequent sections. This is followed by a presentation of the physics and computational improvements and their effect on the unfolding accuracy. This includes detector response modelling ( $\$ 2.3)$, energy differentiation $(\S 2.4)$, measurement configuration $(\S 2.5)$, photonuclear attenuation $(\S 2.6)$, and non-ideal attenuation conditions $(\S 2.7)$.

\subsection{New functional form}

One of the methods to tame the ill-posed problem of spectral unfolding is to specify the spectrum using a functional form with only a few free parameters. Compared with the other methods $(\S 1.7)$, spectrum parameterization has the advantages of compact specification of the spectrum, and the potential for true robustness with standard leastsquares minimization without requiring unrealistic measurement accuracy or a-priori knowledge of the spectrum. Parameterization does not restrict the spectrum any more than the smoothing or regularization constraints do in the bin-by-bin iterative unfolding methods, provided that the functional form used is accurate, flexible and robust. 
Ideally, a functional form should satisfy the following six conditions. It should (a) be relatively simple so that its behaviour is easily understood; (b) have as few free parameters as reasonable to be robust during spectral unfolding; $(c)$ be based on physics to prevent non-physical shapes; (d) be flexible enough to accommodate the diversity of current clinical spectra; (e) clearly characterize the endpoint energy, which has been difficult to unfold; and, (f) not require a-priori knowledge of the spectrum or the linac head. To meet these conditions, a new function is proposed below. A comprehensive benchmark set of diverse, validated, high precision Monte Carlo spectra is generated and used to evaluate the performance of the proposed function and to compare that performance to existing functions from the literature. The benchmark set has 65 clinical and research spectra from 3.5 MV to $30 \mathrm{MV}$. The clinical spectra are for the Varian, Elekta, Siemens, Tomotherapy and Cyberknife linacs. The research spectra are for the linacs of the National Research Council of Canada (NRC) and the National Physics Laboratory (NPL) in the UK. The set includes clinical spectra on- and off-axis, with a flattening filter (WFF), flattening-filter free (FFF), and in treatment and imaging modes. App. A contains more description of the spectra in the benchmark set.

The proposed function (Eq. 2.1, p. 17) is constructed as follows. Let $C$ be a free parameter, $E$ the photon energy, $E_{e}$ the incident electron kinetic energy, and $E_{t}$ its total energy. Photons are assumed to be produced in the front thin layer of the target with an energy fluence spectrum $\psi_{\text {thin }}(E)$. The photons are then attenuated by two materials representing the target (high $\mathrm{Z}$ ) and the aggregate of beam-modifying devices (low/medium Z), with their effective thicknesses as free parameters, $C_{1}^{2}$ and $C_{2}^{2}$, respectively. The square used with the free parameters is to ensure positivity, which is found to be necessary for unfolding robustness. The fit quality to the benchmark set is found to be insensitive to the exact choice of the two materials, hence $\mathrm{W}$ and $\mathrm{Al}$ are used. 
The devised thin-target spectrum, $\psi_{\text {thin }}(E)$, uses a combination of the patterns that are common to different thin-target formulae. The alternative of using a specific formula is found to be unjustifiably restrictive for a function that is intrinsically phenomenological. The thin-target formulae considered are those for bremsstrahlung production integrated over all photon emission angles, rather than those for forward-directed photon production. This seems counter-intuitive at first glance, but all-angle formulae are found to be more appropriate for two reasons: (a) the angular spread of the incident electrons is much larger than that for the produced photons and can safely be assumed isotropic; therefore an integral over all electron angles (even if one is only interested in their contribution to forward photon production) becomes an integral over photon emission angles; ${ }^{8}$ and, (b) the function should be flexible enough to handle off-axis spectra and spectra averaged over large fields (for possible use in applications other than transmission analysis), and thus an all-angle formula is more appropriate. All-angle formulae over-estimate the photon lower-energy component in the forward direction because in reality lower-energy photons are more isotropic than higher-energy ones. However, this over-estimation is naturally compensated for in three ways. (a) Lower-energy photons are over-attenuated by the full target thickness because in reality they are produced deeper in the target; (b) Lower-energy photons are preferentially eliminated by the flattening filter (if it exists); and, (c) The beam softening due to scatter by the target and/or the flattening filter is ignored.

Observing the common patterns in all-angle formulae ${ }^{24}$ indicates that most of those formulae contain the three terms: $1, \frac{E_{t}-E}{E_{t}}$ and $\left(\frac{E_{t}-E}{E_{t}}\right)^{2}$. The first and third terms almost always have a fixed ratio to each other, while the second is scaled by various constants, approximate screening functions and Coulomb corrections. Therefore it is justifiable to include the parameterized term $\left[1+C_{1}^{\prime} \frac{E}{E_{t}}+\left(\frac{E}{E_{t}}\right)^{2}\right]$ in the proposed function. Similarly, the term $\frac{E_{t}\left(E_{t}-E\right)}{E}$ (a bremsstrahlung impact parameter) appears

\subsection{NEW FUNCTIONAL FORM}


with various screening functions and Coulomb corrections added to it inside and outside a logarithmic function, therefore multiplying the square bracket just mentioned by $\left[\ln \left(\frac{E_{t}\left(E_{t}-E\right)}{E}+C_{3}^{\prime}\right)+C_{2}^{\prime}\right]$ is reasonable. Replacing $E_{t}$ with $E_{e}$ is found to have virtually no effect on fit quality, and it is thus adopted for simplicity; i.e., the function includes $\left[1+C_{1}^{\prime \prime} \frac{E}{E_{e}}+\left(\frac{E}{E_{e}}\right)^{2}\right]\left[\ln \left(\frac{E_{e}\left(E_{e}-E\right)}{E}+C_{3}^{\prime \prime}\right)+C_{2}^{\prime \prime}\right]$. Assuming no energy spread of the incident electron beam, the physical condition $\psi\left(E_{e}\right)=0$ can be imposed, which requires that $C_{3}^{\prime \prime}=\exp \left(-C_{2}^{\prime \prime}\right)$. Fits to the full benchmark set show that $C_{2}^{\prime \prime}=-0.5$ (and consequently $C_{3}^{\prime \prime}=1.65$ ) achieve the best overall fit quality. These values for $C_{2}^{\prime \prime}$ and $C_{3}^{\prime \prime}$ are used in the proposed function, and they can be thought of as average nuclear screening and/or Coulomb correction factors. Based on this discussion, the proposed function has the four free parameters $C_{1}, C_{2}, C_{3}$ and $E_{e}$, it goes to zero at $E=E_{e}$, and it is given by

$$
\begin{gathered}
\psi(E)=\psi_{\text {thin }}(E) \exp \left[-\mu_{W}(E) C_{1}^{2}-\mu_{A d}(E) C_{2}^{2}\right] \\
\psi_{\text {thin }}(E)=\left[1+C_{3} \frac{E}{E_{e}}+\left(\frac{E}{E_{e}}\right)^{2}\right]\left[\ln \left(\frac{E_{e}\left(E_{e}-E\right)}{E}+1.65\right)-0.5\right]
\end{gathered}
$$

The function can be refined further using additional free parameters for the contribution of the $511 \mathrm{keV}$ peak from positron annihilation events, and for the electron beam energy spread. The contribution of the $511 \mathrm{keV}$ peak can be modelled using a Dirac delta function with a free parameter for its amplitude. The electron beam energy spread (which makes the maximum photon energy, $E_{m}$, larger than the mean incident electron kinetic energy, $E_{\epsilon}$ ) can be modelled using a superposition of a finite number of spectra related by a free parameter that models the energy spread. These additional free parameters can be extracted with direct fitting of the spectrum to the proposed form. However, they cannot be unfolded from transmission measurements because their effect on the measured signals is very small.

\subsection{NEW FUNCTIONAL FORM}


Eq. 2.1 is fitted to each energy fluence spectrum in the benchmark set using the Levenberg-Marquardt least-squares algorithm. ${ }^{70}$ For the full benchmark set of 65 spectra, the average root-mean-square (RMS) energy fluence deviation between the fitted and the original spectra, normalized to the mean energy fluence of each spectrum is $1.7 \%$ ( $1.5 \%$ if the $511 \mathrm{keV}$ peak is modelled). The average absolute deviation between the fitted incident electron energies and those used in the Monte Carlo simulation that generated the spectra is $1.4 \%$. This excellent ability to directly fit spectra and incident electron energies is a prerequisite for accurate unfolding of these quantities from transmission data. For completeness, the corresponding deviations in the maximum, most probable and average photon energies are, respectively, $4.3 \%, 3.9 \%$ and $0.6 \%$.

Fig. 2.1 demonstrates the overall excellent ability of the function to fit different classes of spectra: panel (a) for spectra of linacs with different head designs; panel (b) for spectra of a given linac on- and off-axis, WFF and FFF; panel (c) for spectra with different incident electron beam energy spread (Gaussian with 3\% FWHM for Varian $4 \mathrm{MV}, 17 \%$ FWHM for Elekta $6 \mathrm{MV}$ and 14\% FWHM for Siemens $6 \mathrm{MV}$ ); panel (d) for research spectra which are different from typical clinical spectra; panel (e) for very different spectra created from the same electron beam but with different filtration conditions (14 cm of $\mathrm{Al}$ added); and, panel (f) for the treatment and imaging spectra of linacs that are dedicated to intensity modulated radiation therapy (IMRT). The differences between the original spectra and their functional fits lead to systematic errors in the smallest calculated transmission data with typical and maximum values of 0.1 and $0.2 \%$, respectively. Therefore the function can be deemed accurate and flexible for unfolding spectra from transmission measurements, in addition to its general uses. The function is also found to be robust (i.e., not over-parameterized) when used in spectral unfolding from transmission data. Report I contains the graphical fits of the proposed function to the full benchmark set, along with the corresponding fit coefficients. 


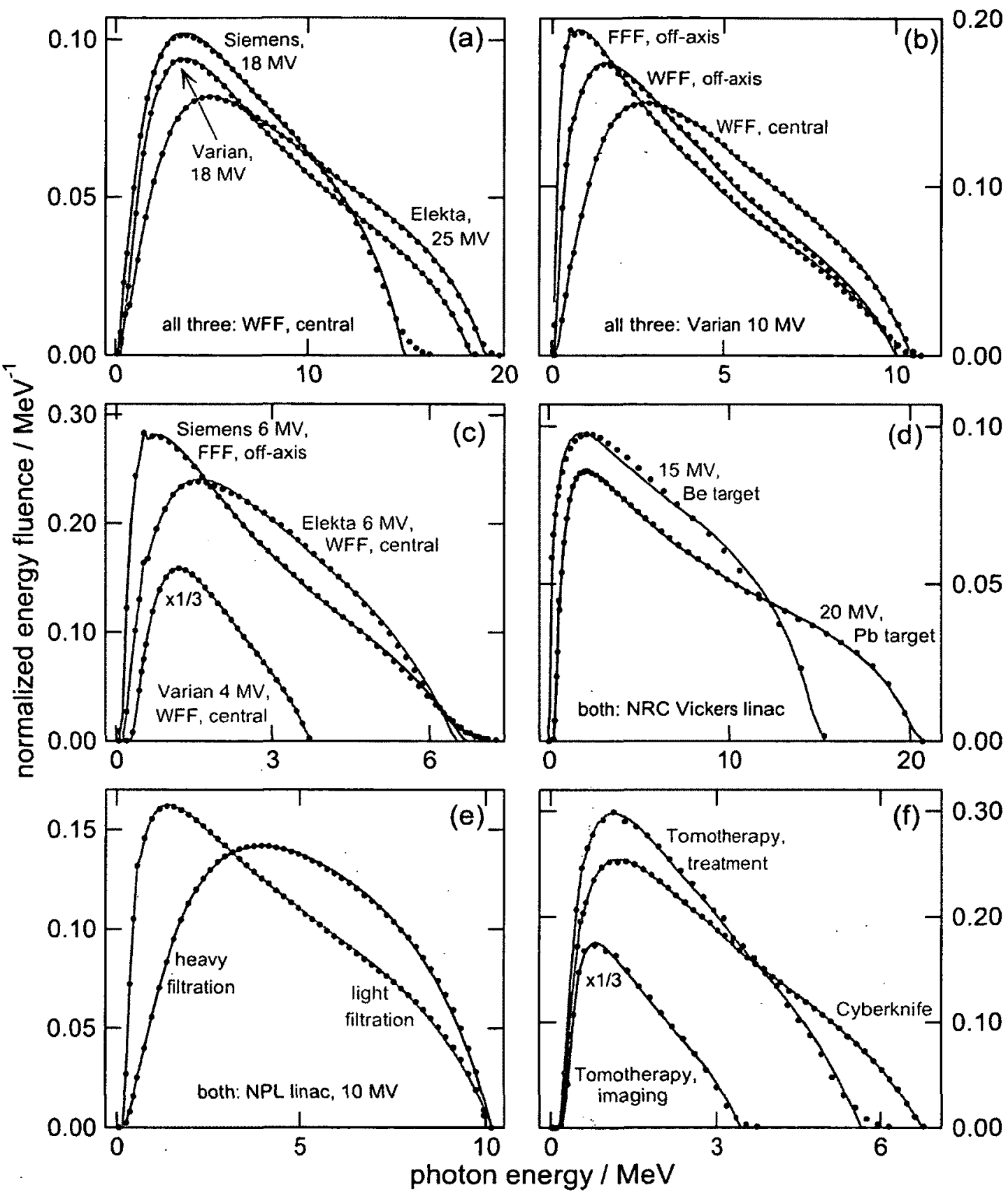

Figure 2.1: Fits of the proposed function (with the $511 \mathrm{keV}$ contribution included) to 16 Monte Carlo spectra from the benchmark set of 65 spectra. The dots are the original spectra and the solid lines are the fits. The fits are typical of others. The terms 'central', 'off-axis', 'WFF' and 'FFF' refer to, respectively, a centralaxis spectrum, an off-axis spectrum, with flattening filter, and flattening-filter free. For graph clarity, only every other original Monte Carlo point is shown, and one spectrum in each of panels (c) and (f) is scaled down by a factor of 3 . Spectra are normalized to unit energy fluence. From Paper I: 
The literature has existing functional forms that were used in various contexts to specify photon spectra. In App. B, a compilation of 11 previous functions is presented, along with the results of testing their accuracy and flexibility using the benchmark set of 65 spectra. Overall, the proposed function outperforms the existing functions in terms of the combination of accuracy, flexibility and robustness.

\subsection{Unfolding}

For the theoretical studies presented in this chapter, transmission data are generated computationally using Eq. 1.1 (p.8) for different measurement configurations using one or more attenuators and detectors. The data are smeared with computational Gaussian noise for some investigations. The input transmission data from different attenuators and/or detectors are fed simultaneously to the unfolding algorithm. During the minimization, transmission data are calculated using Eq.1.1, and the energy fluence is specified using Eq. 2.1 (p.17), with $C_{1}, C_{2}, C_{3}$, and $E_{e}$ as free parameters. The Levenberg-Marquardt least-squares minimization algorithm ${ }^{70}$ is used, and it requires analytical first order derivatives of $T_{i d e a l}$ of Eq. 1.1 with respect to each free parameter. The minimized objective function is the standard $\chi^{2}$, the sum of the squares of the inverse-variance-weighted difference between the input transmission data and the transmission data calculated from Eq. 1.1 using the estimated free parameters in a given iteration. The integral in Eq. 1.1 is evaluated using Gauss-Legendre quadrature of order 200 to limit the integration errors to less than $0.01 \%$. The lower-energy bound, $E_{l}$, is always $10 \mathrm{keV}$. The interpolation for the pre-calculated detector energy response ( $\$ 2.3)$ is linear in $R(d, E)$ versus $\ln (E)$, and for the mass attenuation coefficient it is linear in $\ln \frac{\mu}{\rho}(E)$ versus $\ln (E)$. Typical deviations between the input and fitted transmission data are of the order of the corresponding Gaussian noise, and the $\chi_{\min }^{2}$ per degree of freedom is of the order of unity. Minimization takes very few seconds. 


\subsection{Accurate detector energy response modelling}

Accurate modelling of the detector energy response per unit energy fluence, $R(d, E)$ in Eq. 1.1 (p. 8), is a prerequisite for accurate spectral unfolding, particularly when data from more than one detector are used. In previous studies, the most common approximation is that the material of the ion chamber wall and buildup cap are airequivalent, thus the air-cavity dose, $D_{c a v}$, was assumed to be equal to the collision airkerma, $K_{c o l}^{a i r}$, and consequently $R(d, E)$ is proportional to $\left(\mu_{e n} / \rho\right)^{a i r}$, the mass energy absorption coefficient for air. ${ }^{46,47,49,52,66,71,72}$ Some studies improved this approximation by either measuring the response at a few known low energies (e.g., ${ }^{137} \mathrm{Cs}$ and ${ }^{60} \mathrm{Co}$ ) and interpolating or extrapolating at other energies, ${ }^{50,53,59,60}$ or by using approximate expressions to account for the deviation from air equivalence. ${ }^{44,48,51}$

The energy response of the detector used in this chapter is calculated using the EGSnrc usercode cavity, ${ }^{39}$ and validated experimentally $(\S 4.5)$. The detector is an Exradin A19 Farmer-type chamber (Fig. 2.2a). The chamber is fitted with one of three buildup caps made of, respectively, polymethylmethacrylate (PMMA), Al or a W alloy with $90 \% \mathrm{~W}, 5 \% \mathrm{Ni}$ and $5 \% \mathrm{Cu}$. The caps have wall thicknesses roughly equal to the range of $10 \mathrm{MeV}$ electrons in their respective materials. The irradiation geometry used for the energy response calculations is shown in Fig. 2.2b. Calculations are done at 40 energies from $100 \mathrm{keV}$ to $35 \mathrm{MeV}$, equi-spaced in $\ln (E)$. The kinetic energy thresholds for the production and transport of charged particles and photons are $10 \mathrm{keV}$. The statistical uncertainty is kept below $0.1 \%$ so that its effect on the uncertainty of the unfolded spectra is negligible.

The calculation results shown in Fig. 2.2c indicate that the commonly used assumption of the constancy of $D_{c a v} / K_{c o l}^{a i r}$ with energy is not satisfied, even for low-Z caps which are closest to being air-equivalent. The variation in this ratio, relative to its mean

\subsection{ACCURATE DETECTOR ENERGY RESPONSE MODELLING}


(a)

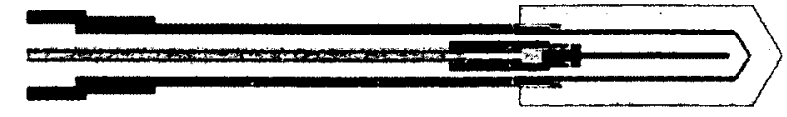

point source

(b)

(monoenergetic)
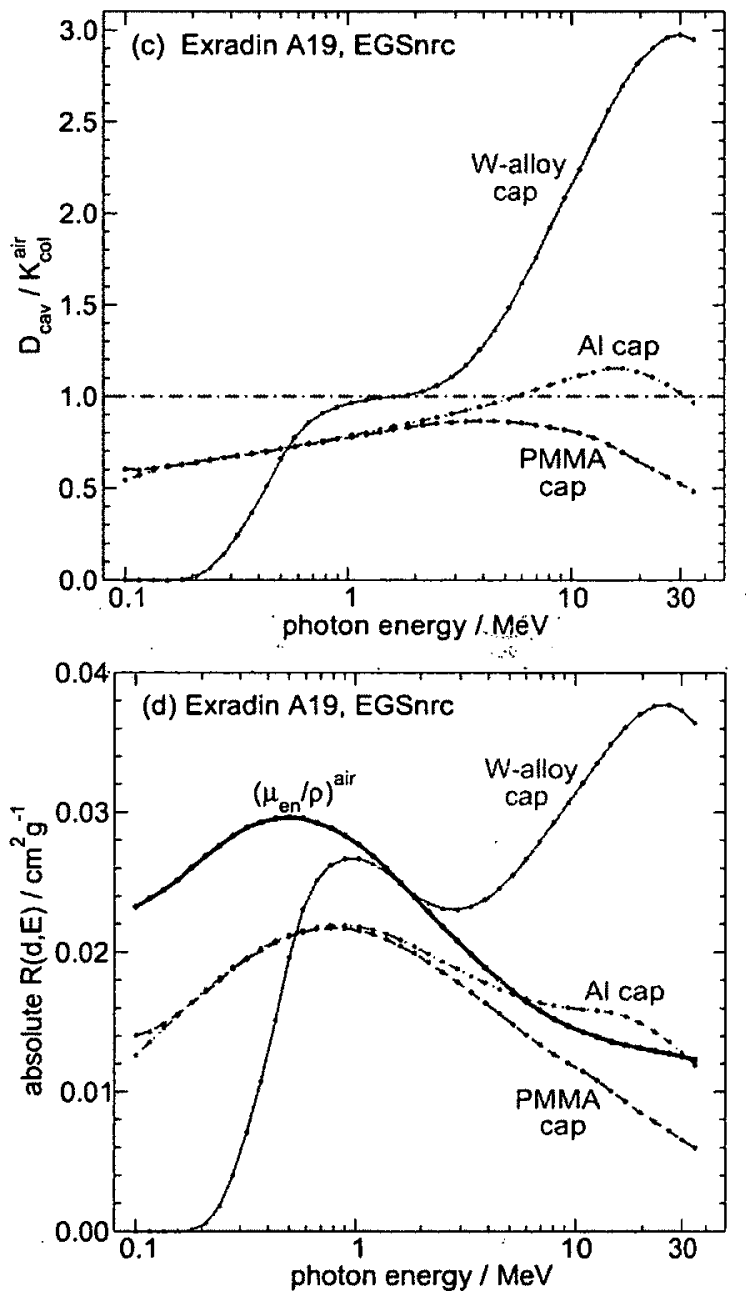

Figure 2.2: (a) egs ++ model $^{73}$ of an Exradin A19 ion chamber (from blueprints) fitted with a W-alloy buildup cap. (b) Irradiation geometry for the energy response calculations (not to scale). (c) Air-cavity dose, $D_{c a v}$, per unit collision air-kerma, $K_{c o l}^{a i r}$. (d) Air-cavity dose per unit energy fluence. For comparison, $\left(\mu_{e n} / \rho\right)^{a i r}$ is also shown. The ratio $R(d, E) /\left(\mu_{e n} / \rho\right)^{a i r}$ from panel (d) gives the respective data in panel (c). From Paper II.

\subsection{ACCURATE DETECTOR ENERGY RESPONSE MODELLING}




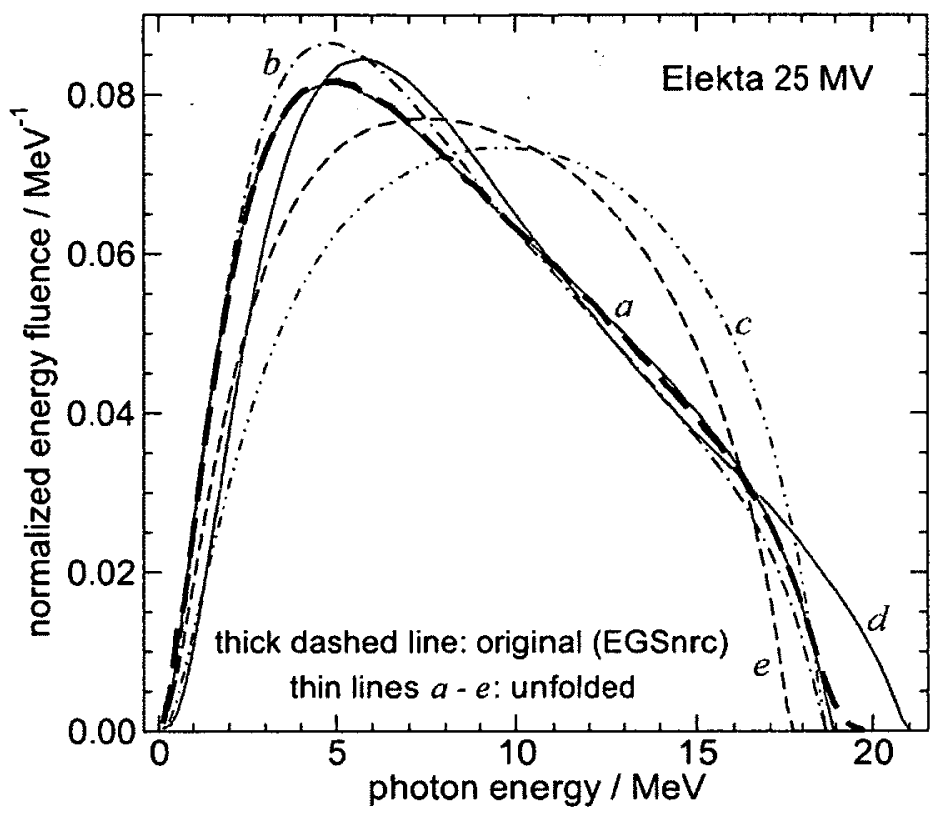

Figure 2.3: Effect of approximating the detector energy response on the accuracy of the unfolded spectra. Spectra are normalized to unit energy fluence. See text for the details of curves $a$ to $e$. From Paper II

value over the energy range shown, is $\pm 26 \%, 35 \%$ and $120 \%$ for the $\mathrm{PMMA}, \mathrm{Al}$ and W-alloy caps, respectively. The corresponding values of $R(d, E)$ are shown in Fig. $2.2 \mathrm{~d}$, along with their commonly used approximation as $\left(\mu_{e n} / \rho\right)^{a i r}$.

To demonstrate the effect of this approximation on the accuracy of the unfolded spectra, an Elekta $25 \mathrm{MV}$ spectrum is used as a point source to generate transmission data using Eq. 1.1 (p. 8) with different attenuators (C and $\mathrm{Pb}$ ) and different detectors (the A19 chamber, once with the PMMA cap and once with the W-alloy cap). The data are generated using the correct $R(d, E)$ values from Fig. $2.2 \mathrm{~d}$. No noise is added to the data to be able to isolate the effect being investigated. Spectral unfolding is performed using input transmission data sets with different attenuator/cap combinations, and the results are shown in Fig. 2.3. When the correct $R(d, E)$ for all the detectors involved is used during the minimization, the unfolded spectrum is curve $a$, regardless of which input transmission data sets are used. This is because the data are noise-free and the

\subsection{ACCURATE DETECTOR ENERGY RESPONSE MODELLING}


minimization is completely robust. Curve $a$ is almost identical to the original spectrum, which validates the unfolding mechanics and demonstrates the flexibility of the functional form. When $R(d, E)$ for all the detectors involved is approximated as $\left(\mu_{e n} / \rho\right)^{a i r}$ during the minimization, the unfolded spectra for input transmission data with different [attenuator/cap] combination are curves $b$ for [C/PMMA], $c$ for [C/W-alloy], $d$ for [C/PMMA + C/W-alloy], and $e$ for [C/PMMA + C/W-alloy + Pb/PMMA + Pb/Walloy]. The error in the unfolded spectrum is larger when some or all of the input transmission data involve a high- $Z$ cap because the approximation of the detector response is worse. Overall, the significant effect of the accuracy of the detector response model on the accuracy of the unfolded spectrum is evident.

\subsection{Improving energy differentiation}

The most fundamental way to improve the unfolding robustness is to increase the energy differentiation in the input transmission data. In this study, two methods are proposed to achieve this: combining transmission data measured using multiple detectors of very different energy response, and combining transmission data from multiple attenuators of very different atomic numbers. These two methods are discussed here.

Detectors: Fig. 2.2d (p.22) shows that the ion chamber with a high-Z W-alloy cap responds significantly more to higher-energy photons than it does to lower-energy ones. The same chamber with a low-Z PMMA cap exhibits an opposite trend. Therefore if some of the measurements are made with a high- $\mathrm{Z}$ cap while the rest are made with a low- $Z$ cap, the spectral information in the combined data will be more than the that from typical transmission measurements in previous studies which used an ion chamber with one generic cap for buildup. In other words, different $R(d, E)$ can be used as spectral weighting functions to improve energy differentiation. The large steady increase in response at high energies for the chamber with a high- $Z$ cap is particularly 
appealing because it amplifies the slow variation of the attenuation coefficient in that energy range. The proposal of using multiple detectors with different energy responses can be generalized to physically-different detectors. However, using one chamber with two caps of very different atomic numbers has the advantages of experimental simplicity and consistency in the combined data, without having to deal with differential detector effects.

Attenuators: To avoid degeneracy of the solution of the unfolding problem, the mass attenuation coefficient, $\mu / \rho$, of the attenuator material must be monotonic with energy i.e., the minimum $\mu / \rho$ must occur at an energy larger than the maximum photon energy of the spectrum. Therefore in previous studies high- $\mathrm{Z}$ materials [e.g., $\mathrm{Pb}$ with $(\mu / \rho)_{\min }$ at $\sim 2.5 \mathrm{MeV}$ ] were deemed unsuitable as attenuators for most therapy beams, and only a single low- or medium-Z attenuator was used [e.g., $\mathrm{Cu}, \mathrm{Al}$ or water with $(\mu / \rho)_{\min }$ at $\sim 7,19$ and $30 \mathrm{MeV}$, respectively]. However, the slow variation of $\mu / \rho$ with energy for that single attenuator causes the problem to be ill-posed. This study proposes a different approach to the choice of the attenuator materials as follows. Fig. 2.4 shows that past $8 \mathrm{MeV}, \mu / \rho$ changes with energy much more rapidly for $\mathrm{Pb}$ than it does for $\mathrm{C}$ (by up to a factor of 8 at $25 \mathrm{MeV}$ ), with the actual $\mu / \rho$ increasing for $\mathrm{Pb}$ and decreasing for $\mathrm{C}$. Therefore, for a fictitious spectrum with no photons below $8 \mathrm{MeV}$, a high- $Z$ attenuator clearly provides much better energy differentiation than a low- $Z$ one. This suggests that for realistic high-MV beams (e.g., 15-25 MV), if some of the measurements are made with a high- $Z$ attenuator alone while the rest are made with a low-Z attenuator alone to eliminate degeneracy, the spectral information in the combined data will be more than that when only one low- $\mathrm{Z}$ attenuator is used.

\subsection{IMPROVING ENERGY DIFFERENTIATION}




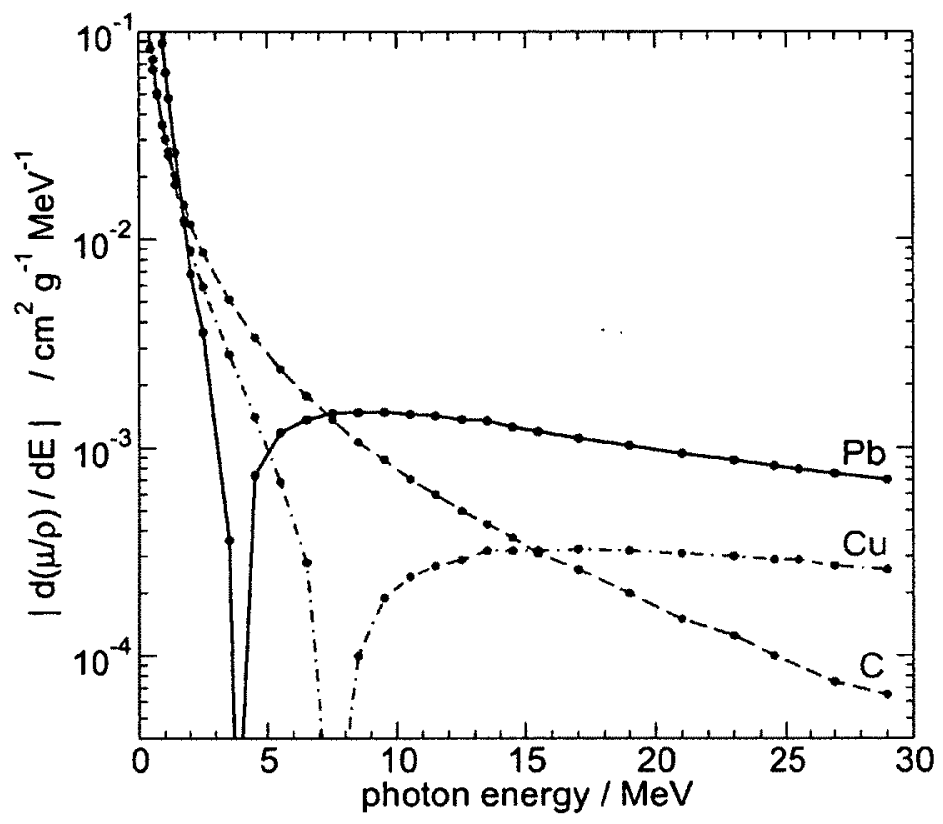

Figure 2.4: Absolute value of the rate of change with energy for the mass attenuation coefficient $(\mu / \rho)$. $\mathrm{Pb}$ provides better energy differentiation than $\mathrm{C}$ below $1.5 \mathrm{MeV}$ and again past $8 \mathrm{MeV}$. Data are also shown for $\mathrm{Cu}$ because it is used for special tests in this study. From Paper II.

\subsection{Optimizing the measurement configuration}

There are many possible variations when using multiple attenuators and detectors. To determine the optimum one, ten configurations are evaluated using transmission data calculated using Eq.1.1 (p. 8) and smeared with simulated experimental noise. For this exercise, the low- $\mathrm{Z} /$ high- $\mathrm{Z}$ attenuators and buildup caps used are $\mathrm{C} / \mathrm{Pb}$ and $\mathrm{PMMA} / \mathrm{W}-$ alloy, respectively. All configurations have the same total number of transmission data points (24 points) and the same transmission cutoff of 0.01 (i.e., $1 \%$ ).

The ten configurations (numbered $\mathrm{C} 1$ to $\mathrm{C} 10$ ) are described here and summarized in the first three columns of Table 2.1 (p.29). In C1 to C4, a separate full transmission curve is calculated for each attenuator/cap combination. In $\mathrm{Cl}$, all the data are for C/PMMA, which is similar to previous studies and its performance is taken as the 
baseline. In $\mathrm{C} 2$, the number of data points is equally split between C/PMMA and $\mathrm{C} / \mathrm{W}$-alloy, which evaluates the concept of using multiple detectors of different energy response. In $\mathrm{C} 3$, the data are split between $\mathrm{C} / \mathrm{PMMA}$ and $\mathrm{Pb} / \mathrm{PMMA}$, which evaluates the concept of using multiple attenuators. In $\mathrm{C} 4$, the data are split between $\mathrm{C} / \mathrm{PMMA}$, $\mathrm{C} / \mathrm{W}$-alloy, $\mathrm{Pb} / \mathrm{PMMA}$ and $\mathrm{Pb} / \mathrm{W}$-alloy, each forming a separate transmission curve with 6 data points, which evaluates the combination of different attenuators and detectors. In C5, only the PMMA cap is used and a single transmission curve is obtained by adding alternating thicknesses of the two attenuator materials (i.e., the first measurement is with $\mathrm{Pb}$, the second adds the same mass thickness of $\mathrm{C}$, the third adds $\mathrm{Pb}$, etc) with the rationale that it may provide better sampling of the spectrum. C6 is the same as $\mathrm{C} 5$ but the data are split between the two caps (12 points each). In $\mathrm{C} 7$, only the PMMA cap is used, with $\mathrm{Pb}$ used up to an arbitrary transmission value of 0.5 , then the remaining attenuation is done with $\mathrm{C}$ (to be compared with the optimum switch of attenuators in $\mathrm{C} 9$ below). $\mathrm{C} 8$ is the same as $\mathrm{C} 7$ except that the starting attenuator is $\mathrm{C}$ and the switch is to $\mathrm{Pb}$. In $\mathrm{C} 9$, the approach of Huang et $a l^{56}$ is employed where $\mathrm{Pb}$ is used up to an optimum transmission value, $T_{\text {switch }}$, to switch to $\mathrm{C}$ for the rest of the attenuation. The value of $T_{\text {switch }}$ is chosen to maintain maximum change in the average attenuation coefficient per unit transmission. ${ }^{56}$ The approach is applicable only to (very) low-MV beams because $T_{\text {switch }}$ is unity for high-MV beams (i.e., no $\mathrm{Pb}$ is to be used). $\mathrm{C} 10$ is the same as $\mathrm{C} 9$ except that the data are split between the two caps.

Each configuration above is tested for two groups of spectra, a low-MV group and a high-MV group. The rationale for this division is that the optimum configuration may not be the same for both groups due to the shape of variation of $\mu / \rho$ and $R(d, E)$ with energy. The four low-MV spectra are Tomotherapy 3.5 MV (imaging), Varian $4 \mathrm{MV}$ and $6 \mathrm{MV}$, and Siemens $6 \mathrm{MV}$. The four high-MV spectra are Varian $15 \mathrm{MV}$ and $18 \mathrm{MV}$, Siemens $18 \mathrm{MV}$, and Elekta $25 \mathrm{MV}$.

\subsection{OPTIMIZING THE MEASUREMENT CONFIGURATION}


Transmission data, $T$, are generated using Eq. 1.1 (p. 8) for the ten configurations using each of the eight spectra just described. Each data set is smeared 1000 times with noise sampled from Gaussian distributions with the following standard deviations, $\sigma$ : $\sigma=0.15 \% T$ for $0.30<T<1.00, \sigma=0.25 \% T$ for $0.10<T \leq 0.30$, and $\sigma=0.40 \% T$ for $0.01 \leq T \leq 0.10$. These noise levels are based on the uncertainty budget from the experimental measurements $(\S 3.4)$. The noisy transmission data are then used as input for spectral unfolding. For configurations that involve multiple attenuators/detectors, the data are fed simultaneously to the unfolding algorithm. The performance of each configuration is evaluated using two metrics for each group of spectra: (a) the average normalized RMS energy fluence deviation between the unfolded and the original spectra, and (b) the average absolute deviation between the unfolded and the original electron energies.

Table 2.1 shows the overall performance of the different configurations. The following observations are made. No extremely large errors are observed in any configuration, which is due to the accuracy, flexibility and robustness of the proposed functional form. Compared with the commonly used approach (C1), using multiple detectors of very different energy responses (C2), or using multiple attenuating materials of very different atomic numbers (C3) improves the unfolding accuracy for both MV groups by a factor of $\sim 2$. When both concepts are combined (C4), further improvement is achieved. In $\mathrm{C} 4$, the excellent ability to unfold the incident electron energy, $E_{e}$, (within $1.4 \%$ of its correct value) is due to both the improved sensitivity to the higher-energy portion of the spectrum and the design of the functional form that has a clear cutoff at $E_{e}$. Comparing C4 with $\mathrm{C} 1$, the enhanced energy differentiation alone (since everything else is equal) improves the accuracy of the unfolded spectra by a factor of $(7.0+6.2) /(2.3+2.3) \approx 3$, and the accuracy of the unfolded $E_{e}$ by a factor of $(7.1+4.8) /(1.4+1.4) \approx 4$. In C5 and C6, the interleaving of the attenuators does not provide an advantage. $\mathrm{C} 7$ performs better

\subsection{OPTIMIZING THE MEASUREMENT CONFIGURATION}


Table 2.1: The average error in the unfolded energy fluence, $\psi$, and the unfolded incident electron energy, $E_{e}$, for the ten attenuator/detector configurations described in the text. From Paper II.

\begin{tabular}{|c|c|c|c|c|c|c|}
\hline \multicolumn{3}{|c|}{ Measurement configuration } & \multirow{2}{*}{\multicolumn{2}{|c|}{$\frac{\text { Low-MV beams }}{\% \text { error in: }}$}} & \multirow{2}{*}{\multicolumn{2}{|c|}{$\frac{\text { High-MV beams }}{\% \text { error in: }}$}} \\
\hline Index & $\begin{array}{l}\text { Attenuator materials } \\
\text { and how they are used }\end{array}$ & $\begin{array}{l}\# \\
\text { caps }\end{array}$ & & & & \\
\hline $\mathrm{C} 1$ & C full curve & 1 & 7.0 & 7.1 & 6.2 & 4.8 \\
\hline $\mathrm{C} 2$ & & 2 & 4.1 & 3.5 & 3.4 & 1.5 \\
\hline $\mathrm{C} 3$ & $\mathrm{C}$ and $\mathrm{Pb}$ full curves & 1 & 3.0 & 2.2 & 3.4 & 2.3 \\
\hline $\mathrm{C} 4$ & & 2 & 2.3 & 1.4 & 2.3 & 1.4 \\
\hline C5 & $\mathrm{Pb}+\mathrm{C}$ alternating & 1 & 7.6 & 9.8 & 6.4 & 4.2 \\
\hline C6 & & 2 & 3.6 & 2.7 & 4.2 & 2.6 \\
\hline $\mathrm{C} 7$ & $T_{\text {switch }}=0.5: \mathrm{Pb}$ to $\mathrm{C}$ & 1 & 4.9 & 4.4 & 7.1 & 5.9 \\
\hline C8 & $\mathrm{C}$ to $\mathrm{Pb}$ & 1 & 7.7 & 8.6 & 10.6 & 8.1 \\
\hline C9 & optimum $T_{\text {switch }}: \mathrm{Pb}$ to $\mathrm{C}$ & 1 & 3.8 & 3.9 & - & - \\
\hline C10 & & 2 & 2.5 & 1.6 & - & - \\
\hline $\mathrm{C}^{\prime}$ & $\mathrm{C} 4$ but $T_{\min }=0.1$ not 0.01 & 2 & 3.2 & 2.6 & 3.3 & 2.0 \\
\hline
\end{tabular}

than $\mathrm{C} 8$ because in $\mathrm{C} 8$ switching to $\mathrm{Pb}$ as the spectrum hardens introduces degeneracy that is not eliminated by any other data. C9 performs better than C7 for low-MV spectra, indicating that optimizing the transmission value for switching between attenuators improves the unfolding accuracy. The performance of $\mathrm{Cl} 10$ and $\mathrm{C} 4$ is almost the same for low-MV spectra. However, C10 requires experimental overhead ${ }^{56}$ and it is not applicable to high-MV beams. Based on these observations, configuration C4 outperforms all others for both MV groups, and it is thus adopted for the rest of this study for all MV beams. Finally, $\mathrm{C}^{\prime}$ in Table 2.1 indicates that using $\mathrm{C} 4$ with a transmission cutoff of $10 \%$ instead of $1 \%$ still allows for reasonably-accurate unfolding with a more compact setup and without having to deal with the issues associated with small transmission signals. This is made possible by the enhanced energy differentiation, combined with the accuracy and robustness of the functional form. 


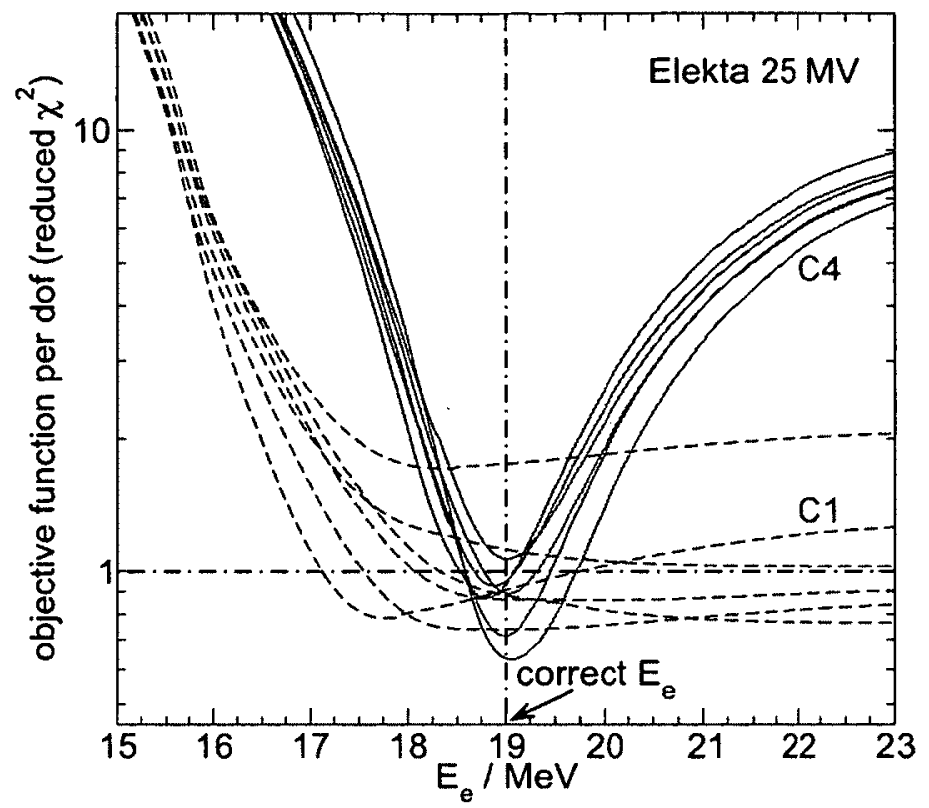

Figure 2.5: Variation of the objective function per degree of freedom (reduced $\chi^{2}$ ) during a grid search of the incident electron energy, $E_{e}$. Data are shown for the optimum configuration proposed in this study (C4) and for the typical configuration in previous studies (C1). The new functional form is used with both $\mathrm{C} 1$ and C4. Different lines represent different sampling of the same noise level. The correct $E_{e}$ is $19.0 \mathrm{MeV}$. From Paper II.

Fig. 2.5 shows the variation of the objective function during a grid search of $E_{e}$ for configurations $\mathrm{C} 1$ and $\mathrm{C} 4$. The objective function has a clear minimum in $\mathrm{C} 4$ compared with $\mathrm{C} 1$. Since the noise levels and the functional form used are the same, the increase in sensitivity to $E_{e}$ is exclusively from the additional spectral information in the input transmission data. However, even with the enhanced sensitivity of $\mathrm{C} 4$, the minima are not identical with different noise sampling, which reflects the inherent ill-conditioned nature of the problem. That $\chi_{\min }^{2} \approx 1$ indicates that Eq. 1.1 (p.8) models the input transmission data in accord with the variance which, in turn, implies that the functional form used for $\psi$ inside the integral is not over- or under-parameterized.

Fig. 2.6 shows an example of the $95 \%$ confidence bounds on the unfolded spectrum for $\mathrm{C} 1$ and $\mathrm{C} 4$. For a given energy, the bounds are estimated as twice the standard deviation of the 1000 unfolded energy fluence values at that energy. The bounds are 


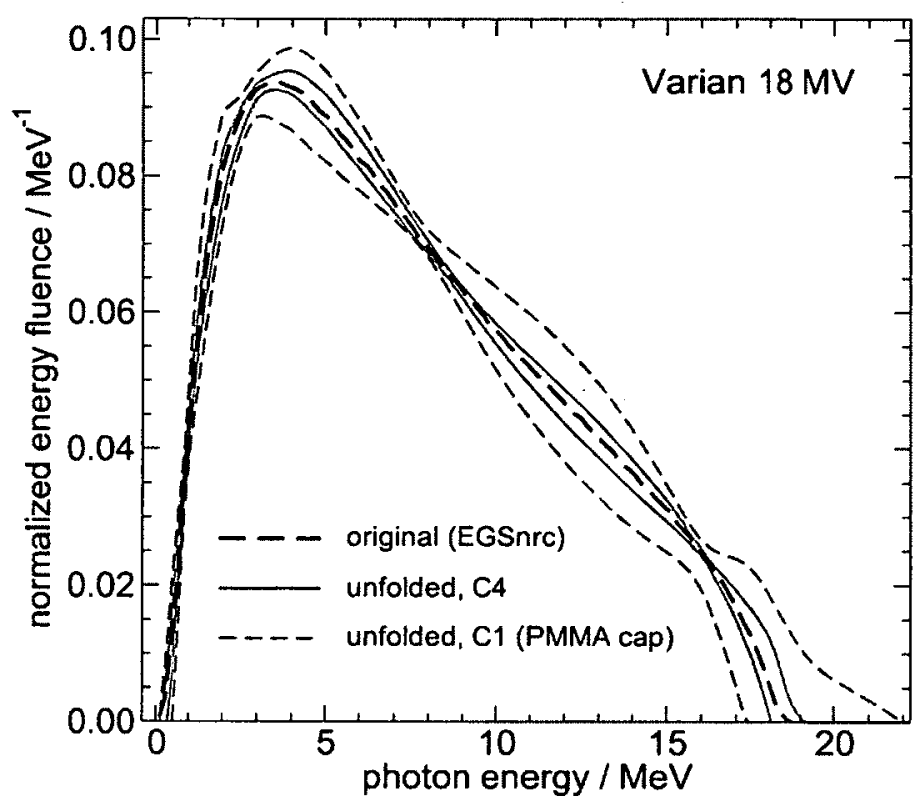

Figure 2.6: An example of the $95 \%$ confidence bounds on the unfolded spectrum for the optimum configuration proposed in this study (C4) and for the typical configuration in previous studies (C1). The new functional form is used with both $\mathrm{C} 1$ and C4. From Paper II.

much tighter with $\mathrm{C} 4$ than they are with $\mathrm{C} 1$, particularly at the higher-energy part of the spectra because it is the part that benefitted the most from the enhanced spectral sensitivity. The bounds for both $\mathrm{C} 1$ and $\mathrm{C} 4$ are tighter at the lower photon energy part because of the stronger variation of $\mu / \rho$ with energy. The irregular shape of the bounds is a result of the shape of the functional form where the unfolded spectra with different noise cross each other more often at certain energies than they do at others, and thus the bounds at those energies are tighter than the bounds at neighbouring energies. The improvements in Figs. 2.5 and 2.6 are in addition to the improvements from using the new functional form and the other new methods of $\S 2.3, \S 2.6$ and $\S 2.7$.

\subsection{Photonuclear attenuation}

Photonuclear cross sections have a resonance from a few $\mathrm{MeV}$ to tens of $\mathrm{MeV}$, and they contribute a few per cent to the total photon cross sections. Their effect on the $\mu / \rho$ val- 


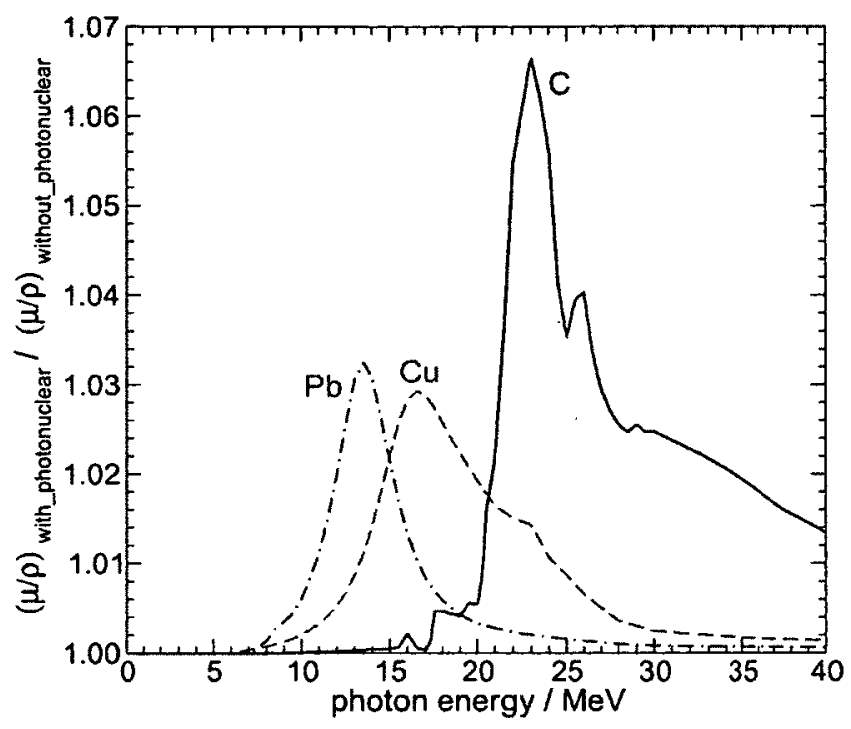

Figure 2.7: For the attenuators used in this study, the increase in the total photon cross section when IAEA photonuclear data are added to the NIST XCOM data. From Paper V.

ues used in Eq. 1.1 (p.8) during the unfolding has been ignored in previous studies. To investigate this effect for the attenuators used in this study $(\mathrm{C}, \mathrm{Cu}$ and $\mathrm{Pb})$, the total photonuclear cross sections for the natural elements are calculated from the cross sections of their constituent isotopes in the evaluated IAEA compilations. ${ }^{36}$ The effect of the photonuclear component on the total cross section is shown in Fig. 2.7. Three high-MV spectra (Varian $18 \mathrm{MV}$, Elekta $25 \mathrm{MV}$ and Vickers $30 \mathrm{MV}$ ) are used in Eq. 1.1 to calculate transmission data, down to $1 \%$, using $\mu / \rho$ values once with and once without the photonuclear component. Fig. 2.8 shows that the effect can be very large (up to $7.5 \%$ ), depending on the beam energy and the attenuator material. For the two clinical beams (Varian and Elekta), the effect is more relevant for $\mathrm{Pb}$ since it has a lower photonuclear threshold. Finally, the noise-free transmission data of the Elekta $25 \mathrm{MV}$ spectrum are used as input to the unfolding, and the $\mu / \rho$ values used during the unfolding are once without and once with the photonuclear component. Fig. 2.9 shows that the photonuclear component has a non-negligible effect on the unfolding accuracy for high-MV beams. The effect is amplified for noisy data.

\subsection{PHOTONUCLEAR ATTENUATION}




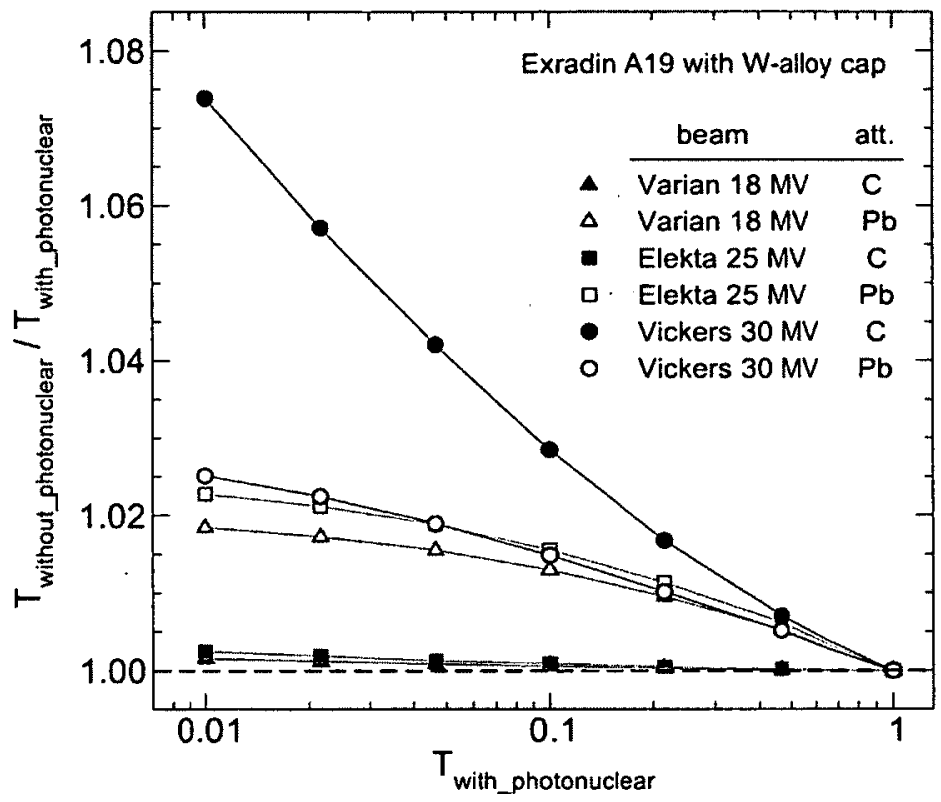

Figure 2.8: Effect of ignoring the photonuclear component on the accuracy of the transmission values, $T$, calculated using Eq. 1.1 (p. 8).

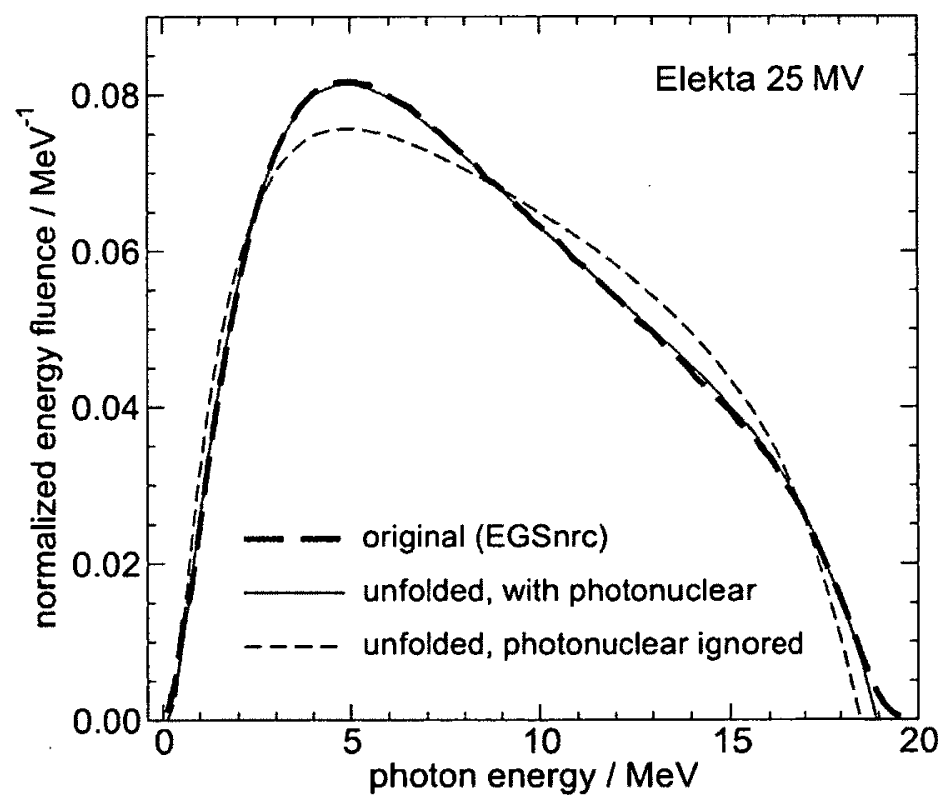

Figure 2.9: An example of the effect on spectral unfolding accuracy when photonuclear cross sections are ignored in $\mu / \rho$ of Eq. 1.1 (p. 8). Spectra are normalized to unit energy fluence. Input transmission data are noise-free to isolate the effect. 


\subsection{Correction for non-ideal attenuation}

Consider a 'best realistic' measurement setup as shown in Fig. 2.10. There are many non-ideal effects that make the measured transmission signals, $T_{\text {meas }}$, deviate from the ideal values, $T_{\text {ideal }}$, of Eq. 1.1 (p. 8). Experimental methods ( $\left.\S 3.3\right)$ are used to correct for certain non-ideal effects such as drifts in beam direction, polarity, ion recombination, leakage, and room scatter. Other non-ideal effects are difficult to eliminate or correct for experimentally, and they are more amenable to computational corrections. Examples of such effects are: (a) forward scatter (coherent or incoherent) and positron annihilation in the attenuator, (b) collimator effects caused by interactions within the collimators or by leakage of the primary radiation through their edges, (c) attenuation and scatter by the intervening air, which has its largest effect on the signal with no attenuator (the denominator of Eq. 1.1), (d) wall backscatter, and, (e) possible electron contamination if full buildup is not achieved.

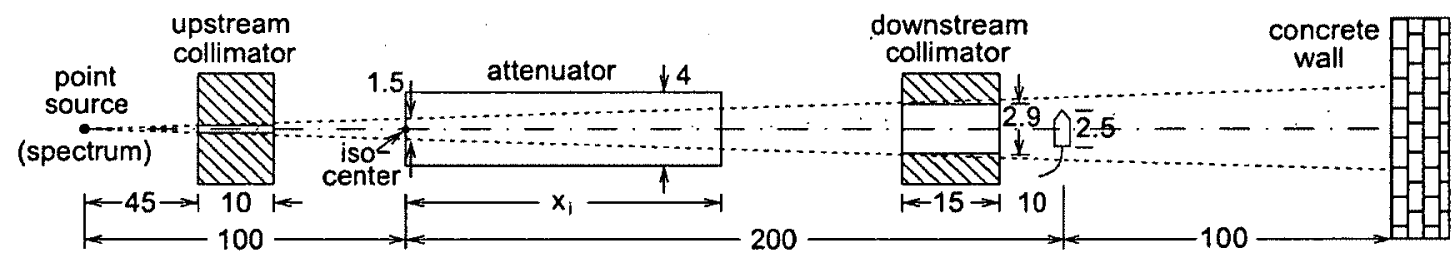

Figure 2.10: A typical transmission measurement setup (dimensions in $\mathrm{cm}$; not to scale). From Paper II.

In this study, the effect of the non-ideal conditions that are not accounted for experimentally is quantified, and methods are developed to correct for them. EGSnrc is used to model the setup of Fig. 2.10 for different point source spectra and different attenuator/cap combinations. Fig. 2.11 shows an example of the energy fluence spectra of the primary, forward-scattered and backscattered photons seen by the detector for the smallest transmission values. The scatter spectra are distinctly different from the 
primary ones. The forward-scattered photons collectively contribute $\sim 1.5 \%$ to the energy fluence experienced by the detector. Backscatter spectra are dominated by two peaks: one at $511 \mathrm{keV}$ from annihilation events, and the other at half of $511 \mathrm{keV}$ from $180^{\circ}$-Compton backscatter. ${ }^{74}$ The contribution of backscattered photons to the energy fluence can be ignored $(\leq 0.01 \%)$.

The effect of non-ideal conditions on transmission data is shown in Fig. 2.12. The effect is up to $1.5 \%$, and its magnitude depends on the beam energy and on the attenuators and detectors used.

Two correction methods are proposed for use during the unfolding, and the two methods are shown to be equivalent. Neither of the two methods requires knowledge of the linac head. The first method is iterative as follows: (a) the experimental transmission data, $T_{\text {meas }}$, are used without correction to unfold an approximate starting spectrum, $\psi$; (b) $\psi$ is used in Eq. 1.1 (p.8) to calculate approximate ideal transmission data, $T_{\text {ideal }}$; (c) $\psi$ is also used as a point source in EGSnrc simulations of the transmission setup to calculate approximate measured data, $T_{E G S n r c}$; (d) a correction factor, $F_{\text {non_ideal }}$, is calculated as $T_{\text {ideal }} / T_{E G S n r c} ;(\mathrm{e})$ the experimental data are corrected by $F_{n o n_{-} i d e a l}$ such that $T_{\text {ideal }}^{\prime}=F_{\text {non_ideal }} T_{\text {meas }}$; (f) $T_{\text {ideal }}^{\prime}$ data are used to unfold a revised spectrum, $\psi^{\prime} ;(\mathrm{g})$ steps b through $\mathrm{f}$ are repeated until the spectrum converges, although it is found empirically that only one iteration is sufficient because $F_{\text {non_ideal }}$ is a ratio of a ratio and thus not very sensitive to the exact spectrum used to calculate it.

It is important to note that if minor discrepancies exist between the $\mu / \rho$ values used in the calculations of $T_{\text {ideal }}$ using Eq. 1.1 and those used in the Monte Carlo calculations of $T_{E G S n r c}$ (due to, e.g., different interpolation grids or formulae, or different data sources), those discrepancies propagate exponentially and can lead to systematic errors of the same order as the effect being investigated here. To avoid this artifact, the

\subsection{CORRECTION FOR NON-IDEAL ATTENUATION}




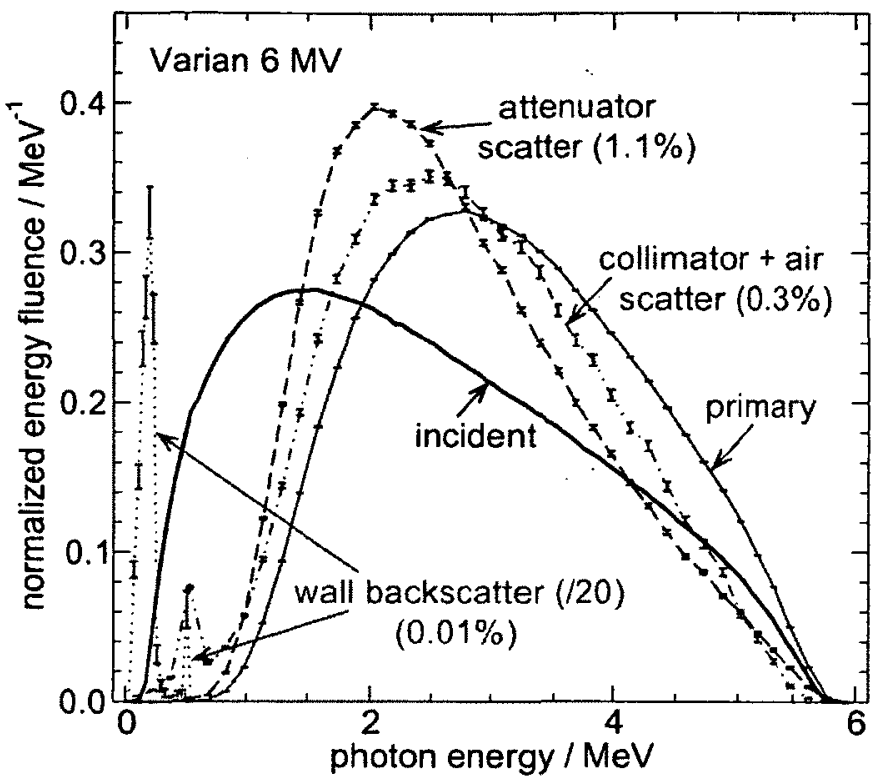

Figure 2.11: EGSnrc spectra of the photons that reach the detector from different sources after the beam is attenuated to $1 \%$ using $\mathrm{Pb}$. Spectra are normalized to unit energy fluence. The spectra of wall backscatter are scaled down for graph clarity. The ratios of the energy fluence from different scatter sources relative to the primary are shown in brackets. From Paper II.

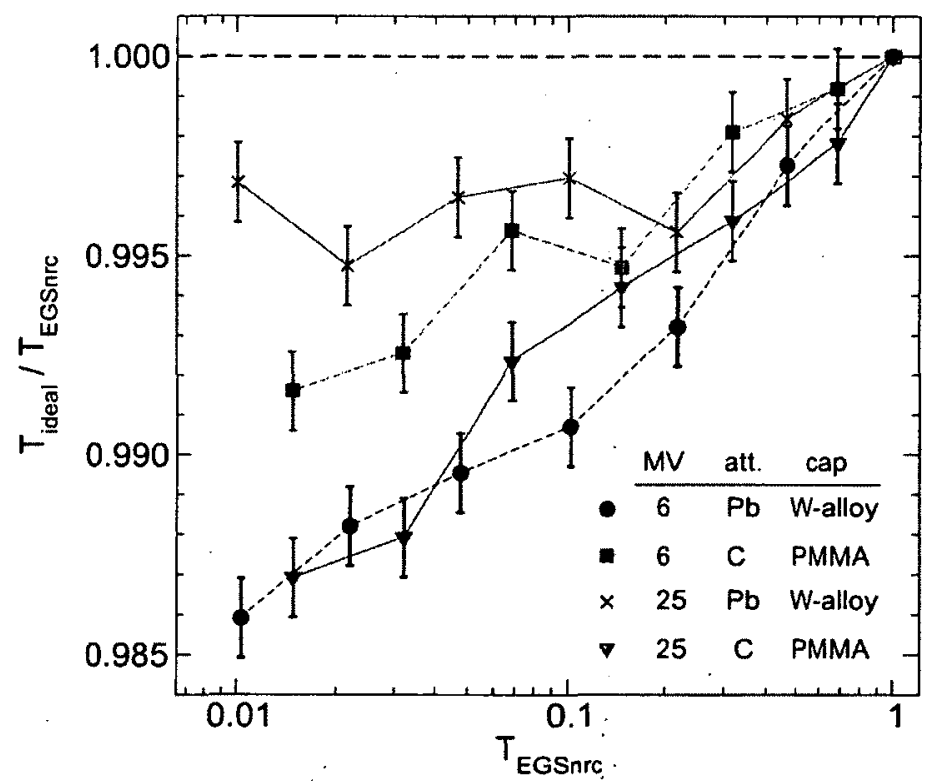

Figure 2.12: Effect of non-ideal attenuation conditions on transmission data. The values of $T_{\text {ideal }}$ are calculated using Eq. 1.1 (p.8) for the Varian $6 \mathrm{MV}$ and Elekta $25 \mathrm{MV}$ spectra. The values of $T_{\text {meas }}$ are calculated using EGSnrc for the same two spectra as point sources in the setup of Fig. 2.10. From Paper II. 


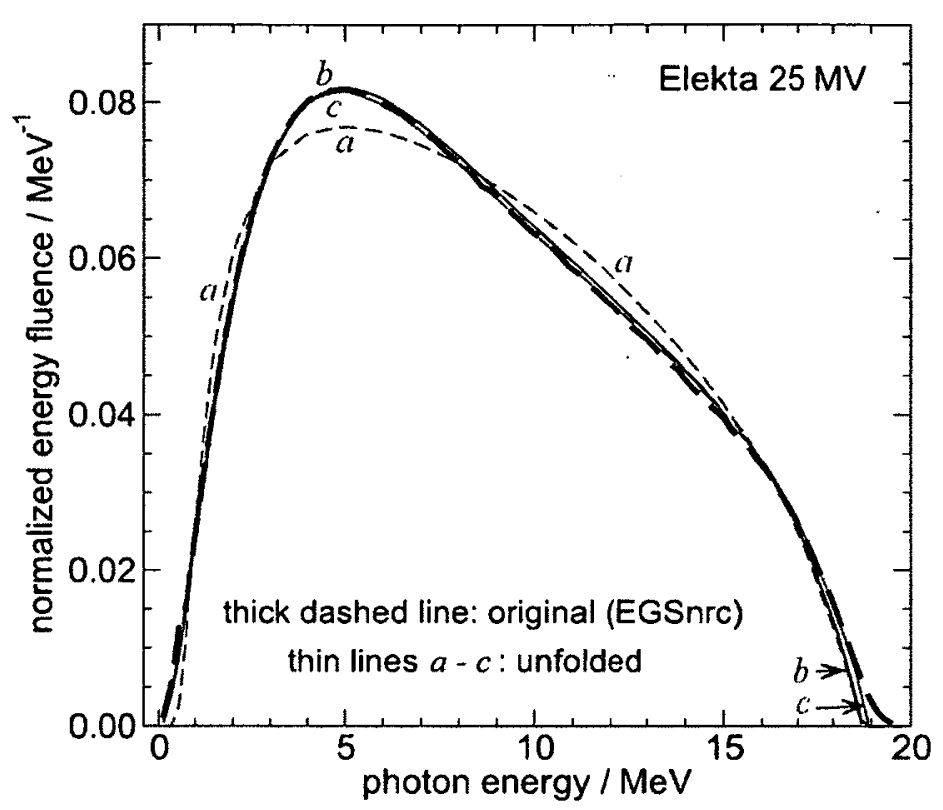

Figure 2.13: The effect of non-ideal conditions on the accuracy of the unfolded spectra. See text for the details of curves $a, b$ and $c$. Spectra are normalized to unit energy fluence. From Paper II.

implementation of XCOM photon cross sections in EGSnrc is refined such that they are consistent with the NIST online database within $0.05 \%$. A summary of this work is given in App. C. All EGSnrc calculations in this study use those refined cross sections.

In the second correction method, EGSnrc is used to generate a full system response matrix. A matrix element, $R\left(d, x_{i}, E\right)$, is the energy response per unit energy fluence of detector $d$ to monoenergetic photons of energy $E$ when the full experimental setup is modelled with an attenuator of mass thickness $x_{i}$. The matrix elements already include the non-ideal effects, therefore no computational correction is required for the measured transmission data. The equivalent of Eq. 1.1 (p. 8) in this case is

$$
T_{\text {meas }}\left(d, x_{i}\right)=\frac{\int_{E_{l}}^{E_{m}} R\left(d, x_{i}, E\right) \psi(E) d E}{\int_{E_{l}}^{E_{m}} R(d, 0, E) \psi(E) d E}
$$

\subsection{CORRECTION FOR NON-IDEAL ATTENUATION}


Fig. 2.13 shows the effect of non-ideal conditions on the unfolding accuracy. When $T_{\text {meas }}$ data (calculated using EGSnrc to mimic a real measurement) are fed to the unfolding algorithm without correction, the unfolded spectrum is curve $a$. For the iterative correction method, curve $a$ is the approximate starting spectrum. After only one iteration, the estimated $F_{n o n_{-} i d e a l}$ is found to be within $0.15 \%$ of its correct value. The unfolded spectrum after the first iteration is curve $b$, and it is already very close to the original spectrum. For the system response matrix method, curve $c$ is the unfolded spectrum. Fig. 2.13 shows that ignoring the corrections for non-ideal conditions reduces the unfolding accuracy, and that the two proposed correction methods are equivalent. For the rest of this study, the iterative correction method is used.

\subsection{Summary}

In this chapter, a physics-based approach is developed which significantly improves the accuracy and robustness of the unfolding problem. The errors in the unfolded spectra caused by the approximations used in previous studies are shown to be very large. A new functional form with four free parameters - one of which is the incident electron energy - is proposed and rigorously validated. Energy differentiation is significantly enhanced by combining data from multiple attenuators and detectors. The configuration with best energy sensitivity is to simultaneously feed the unfolding algorithm with the data of four separate transmission curves using low- $Z$ and high- $Z$ attenuators and a single Farmer chamber, once with a low- $Z$ cap and once with a high- $Z$ cap. The details needed later in this study for the unfolding were generated in this chapter i.e., the detector energy responses, photonuclear attenuation, and correction methods for non-ideal attenuation conditions. Except for the functional form, all the proposed improvements are independent of the unfolding details, and would thus improve the relative performance of any unfolding algorithm. 


\section{Chapter 3}

\section{Experimental measurements}

This chapter presents the transmission measurements performed on the research and clinical linacs at NRC. Most of the chapter is focused on the research linac measurements $(\S 3.1$ - §3.4) because they are used later for benchmarking EGSnrc and for the direct independent validation of the methods presented earlier in Ch. 2. At the end of the chapter (§3.5), the developed experimental methods are demonstrated on a clinical linac, and the differences from the research linac measurements are highlighted.

The ill-conditioned nature of the unfolding problem makes the accuracy of the extracted spectral information strongly dependent on the measurement accuracy. Therefore in the measurements presented here, many influence quantities are investigated and corrected for, and a detailed uncertainty budget for the measured signals is constructed. The resulting rigorous estimates of measurement uncertainties are useful for interpreting differences between measured and calculated transmission data. They also lead to meaningful confidence bounds on the extracted spectral information.

\subsection{The NRC research linac}

The NRC Vickers linac (Fig. 3.1) is a specially-designed linac dedicated to research. It operates at 240 pulses per second, $2.5 \mu$ s each. It produces a horizontal pencil beam of nearly-monoenergetic electrons. Targets of different materials can be placed in the path 


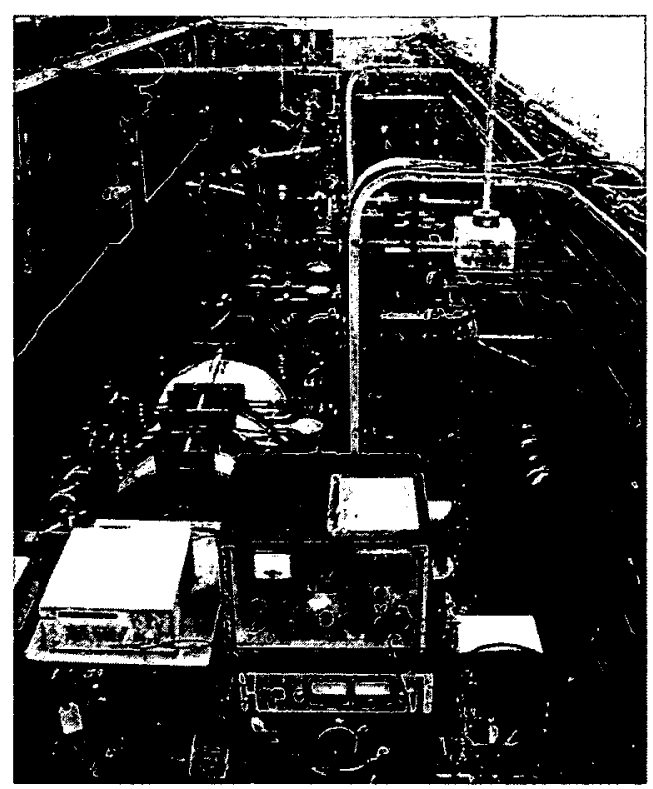

Figure 3.1: The NRC Vickers research linac. Electrons are accelerated towards the far end. From the NRC-IRS website, with permission.

of the electrons to produce bremsstrahlung photon beams. In the past, a $\mathrm{NaI}$ detector was used to measure photon spectra from $\mathrm{Be}, \mathrm{Al}$ and $\mathrm{Pb}$ targets for incident electrons energies from $10 \mathrm{MeV}$ (the lowest stable beam) to $30 \mathrm{MeV}$, in $5 \mathrm{MeV}$ increments. ${ }^{6,11,12}$

Linac electron beams are described using thembination of the electron beam mean energy, energy spread, focal spot size and shape, and angular divergence. For the NRC research linac, the electron energy is known from a bending magnet and slit system that was calibrated using a magnetic spectrometer 68 The estimated standard uncertainty on the electron energy at the exit window is $0.4 \%$. The electron energy spread is known from the physical separation of the slits, and it is approximately Gaussian with a standard deviation of $0.4 \%$. The radial spread is known from detailed radiochromic film measurements, ${ }^{69}$ and it is approximately Gaussian with a FWHM of $1 \mathrm{~mm}$ at the exit window. To determine the angular divergence, Ross et $a l^{69}$ moved the exit window downstream to allow the electron beam to drift an additional $1 \mathrm{~m}$ before acquiring film measurements. Their results indicate a small divergence, taken in this study as $0.03^{\circ}$ with a virtual apex at $1 \mathrm{~m}$ before the exit window: 
Table 3.1: For the research linac, thicknesses of the bremsstrahlung targets used $( \pm 0.01 \mathrm{~cm}$ for $\mathrm{Be}$ and $\mathrm{Al}$ and $\pm 0.003 \mathrm{~cm}$ for $\mathrm{Pb})$. The $\mathrm{Be}$ and $\mathrm{Al}$ targets are cylindrical with $7.26 \mathrm{~cm}$ diameter, and the $\mathrm{Pb}$ targets are squares with $3.0 \mathrm{~cm}$ side lengths. From Paper III.

\begin{tabular}{|c|c|c|c|c|c|}
\hline Target & Density & \multicolumn{4}{|c|}{ Target thickness $(\mathrm{cm})$} \\
\cline { 3 - 6 } material & $\left(\mathrm{g} / \mathrm{cm}^{3}\right)$ & $10 \mathrm{MV}$ & $15,15.7 \mathrm{MV}$ & $20 \mathrm{MV}$ & $30 \mathrm{MV}$ \\
\hline $\mathrm{Be}$ & 1.848 & - & 6.31 & - & - \\
$\mathrm{Al}$ & 2.699 & 2.40 & 3.60 & 4.31 & 6.60 \\
$\mathrm{~Pb}$ & 11.35 & - & 0.793 & 1.016 & - \\
\hline
\end{tabular}

A unique feature of this study is that the measurements are performed on the NRC research linac whose photon spectra were measured using direct spectroscopy, and whose incident electron beam parameters are accurately and independently known. This allows for independent validation of the unfolded spectra in two ways: against the spectra measured with a NaI detector, and against EGSnrc spectra calculated using the known electron beams and geometric setups, with no free parameters. The MV range of the beams that have previous $\mathrm{NaI}$ data $(10-30 \mathrm{MV})$ provides a particularly rigorous validation because the energy dependence of $\mu / \rho$ is weakest for those beams (compared with lower-MV beams), and hence accurate spectral unfolding is most challenging.

On-axis transmission measurements are performed for seven beam/target combinations that have NaI-measured spectra. The nominal beams chosen are 10, 15, 20 and $30 \mathrm{MV}$, with respective measured electron energies of $10.09,15.00,20.28$ and $30.00 \mathrm{MeV}$. Measurements are also made at $15.70 \mathrm{MeV}$ to examine the sensitivity of transmission data to small energy changes (compared with $15.00 \mathrm{MeV}$ ), and to confirm the resolving power of the transmission technique in unfolding the incident electron energy. The bremsstrahlung targets are pure $\mathrm{Be}, \mathrm{Al}$ and $\mathrm{Pb}$, placed $2.1 \mathrm{~cm}$ downstream of the exit window and cooled by forced air. Target thicknesses are given in Table 3.1, and they are sufficient to fully stop the incident electrons. Shielding is added around the targets to reduce stray radiation which could contribute to extra-cameral signals. 


\subsection{Transmission measurement setup}

As part of this project, an experimental setup is designed and built for the measurement configuration that achieves the best energy sensitivity from Ch. 2 - i.e., four separate transmission curves using low- $\mathrm{Z}$ and high- $\mathrm{Z}$ attenuators and a single Farmer chamber, once with a low- $Z$ cap and once with a high- $Z$ cap. The following is a description and justification of the setup components (Fig. 3.2, p. 46 and Fig. 3.3, p. 47).

The narrow-beam geometry starts at the top of Fig. 3.2 (p. 46) with a 10.2-cmthick $\mathrm{Pb}$ collimator touching the target shielding (15 $\mathrm{cm}$ from the exit window), with a non-diverging 1.4-cm-diameter opening. A second 10.2-cm-thick $\mathrm{Pb}$ collimator is placed starting at $50 \mathrm{~cm}$, with a non-diverging $0.77-\mathrm{cm}$-diameter opening. Ion chamber measurements behind the second collimator indicate that the leakage through the collimator is $\sim 0.1 \%$ of the peak signal for the highest MV beam. A PTW7862 monitor chamber, operated at a bias of $300 \mathrm{~V}$ and connected to a Keithley 6517A electrometer, is used to correct the transmission signals for linac output fluctuations. The sensitive volume of the monitor chamber is a central air cylinder of diameter $9.65 \mathrm{~cm}$ and thickness $2.4 \mathrm{~mm}$. The monitor chamber is placed between the two collimators (starting at $27.6 \mathrm{~cm}$ from the exit window), rather than past the second collimator, to allow for a larger signal and to minimize the variable backscatter contribution which would depend on the presence or absence or an attenuator, and on the attenuator material. An NE2581 chamber with

a ${ }^{60} \mathrm{Co}$ buildup cap is used as a field chamber, and the 'field-to-monitor' ratio is used to monitor drifts in the beam direction as discussed below. The field chamber is placed downstream of the monitor chamber between the two collimators such that it falls inside the field of the first collimator but outside the field of the second. This makes the field chamber signal more sensitive to the electrons in the direct field (as opposed to only the scatter component) without obstructing the useful beam. 
The next component downstream is the attenuators. The low- $Z$ attenuator used is graphite, which has not been used in the previous transmission studies referenced throughout this study. Compared with water, it allows for a more compact setup and for lower positioning uncertainties. The pure C used (www.graphitestore.com) is isomolded bars of grade GM10 with a grain size of $10 \mu \mathrm{m}$ to ensure density uniformity. The ash content is $500 \mathrm{ppm}$ and it is assumed to have typical ash composition. ${ }^{75}$ For each MV beam, ten $\mathrm{C}$ bar lengths are used (five for each detector) which successively reduce the signal to $\sim 1.7 \%$ of its value without an attenuator. The bar lengths are integer multiples of the smallest length - multiples of $8.3 \mathrm{~cm}$ for $10 \mathrm{MV}, 9.5 \mathrm{~cm}$ for $15 \mathrm{MV}$ and $11.6 \mathrm{~cm}$ for 20 and $30 \mathrm{MV}$, with respective maximum bar lengths of $83.00,95.00$ and $116.00 \mathrm{~cm}$, all $\pm 0.03 \mathrm{~cm}$. The bars have a square cross section of $3.81 \mathrm{~cm}\left(1.5^{\prime \prime}\right)$ nominal side length, with a milling tolerance of $+0.01^{\prime \prime}$. The extreme case of $a+0.01^{\prime \prime}$ milling error on both side lengths along the full length of a bar would introduce a mass thickness error of $(1.51 / 1.5)^{2}-1=1.3 \%$, which would lead to errors of up to $4 \%$ in the smallest calculated transmission data. To avoid this, the side lengths of each bar are fully mapped in the two orthogonal directions using a spring-loaded digital caliper gauge (Mitutoyo, Denmark) with a resolution of $20 \mu \mathrm{m}$. The data are then used for volume calculations. The mass of the bars is measured using a scale with a resolution of $0.1 \mathrm{~g}$ (Sartorius, Germany). The uncertainty on the individual mass thicknesses, which are later used in the Monte Carlo model, is typically $0.07 \%$. The average density (although not used - see $\S 3.4$ ) is $1.728 \mathrm{~g} / \mathrm{cm}^{3}$ with a sample deviation of $0.4 \%$.

The high- $\mathrm{Z}$ attenuators are pure $\mathrm{Pb}$ rods (www.goodfellow.com). The maximum impurity level is $500 \mathrm{ppm}$, and the typical elemental analysis of the impurities is provided by the supplier. Similar to $C$, ten lengths are used (five per detector) to successively reduce the signal to $\sim 1.7 \%$. The rod lengths are multiples of $0.75 \mathrm{~cm}$ for 10 and $15 \mathrm{MV}, 0.70 \mathrm{~cm}$ for $20 \mathrm{MV}$ and $0.65 \mathrm{~cm}$ for $30 \mathrm{MV}$, with respective maximum 
rod lengths of $7.510,7.000$ and $6.510 \mathrm{~cm}$, all $\pm 0.005 \mathrm{~cm}$ (note that for a given transmission value, higher-MV beams require shorter lengths because $\mu / \rho$ for $\mathrm{Pb}$ has a minimum at $\sim 2.5 \mathrm{MeV}$ ). The rods have a diameter of $1.900 \pm 0.001 \mathrm{~cm}$. Unlike $\mathrm{C}$, it is found more accurate for $\mathrm{Pb}$ to use the combination of average density and physical lengths (compared with individual mass thicknesses) because of minor irregularities in the rods (from dents, sagging, etc). The average density used for all rods is $11.290 \mathrm{~g} / \mathrm{cm}^{3}$, with a sample deviation of $0.15 \%$.

A linear translation system was built to automate the movement of the attenuators (middle and bottom of Fig. 3.2, p.46). Its base is a motorized Velmex bislide, placed perpendicular to the beam axis. It has a travel of $50.8 \mathrm{~cm}$, and a positioning resolution of $5 \mu \mathrm{m}$ (200 steps $/ \mathrm{mm}$ ). The base drives a custom Al support rack that has attenuator slots with center-to-center separation of $5 \mathrm{~cm}$. The attenuators are held using multiple small plates with screws that attach to the rack. The additional scatter caused by the translation system and the side attenuators is negligible ( $\S 3.4)$. The translation system significantly reduces the overhead time during data acquisition, facilitates more randomized repeats, reduces positioning uncertainties, and reduces the uncertainties from beam instability because beam interruption is reduced by a factor of six. The plane of the front surface of the attenuators is placed at $95.1 \mathrm{~cm}$ from the exit window. When the longest $\mathrm{C}$ and $\mathrm{Pb}$ attenuators are placed in the radiation beam, lateral beam scans downstream did not exhibit any profile horns, confirming that the beam is fully intercepted at the back end of the longest attenuators.

$\mathrm{A} \mathrm{Cu}$ rod of length $2.7 \mathrm{~cm}$ and diameter $2.5 \mathrm{~cm}$ is permanently fixed in the middle of the translation system. The transmission signal using the $\mathrm{Cu}$ rod is acquired many times for a given rack of $\mathrm{C}$ or $\mathrm{Pb}$. The 'Cu-to-monitor' ratio has two important uses: short-term second-order corrections for drifts in the beam direction ( $\S 3.3)$, and longterm monitoring of the stability of the energy of the electron beam (§3.4). 
Past the attenuator assembly, a third $\mathrm{Pb}$ collimator is placed starting at $276.5 \mathrm{~cm}$ from the linac exit window. It is 15.3-cm thick, with a non-diverging opening diameter of $2.93 \mathrm{~cm}$. It extends $\sim 15 \mathrm{~cm}$ laterally to reduce large-angle scatter into the detector and to reduce cable irradiation which leads to extra-cameral charge collection.

Transmission signals are acquired $7 \mathrm{~cm}$ downstream from the third collimator using a $0.6 \mathrm{cc}$ Exradin A19 Farmer chamber, operating at $\pm 300 \mathrm{~V}$ and connected to a Keithley 6517A electrometer. A PTW30013 Farmer chamber is used for specific tests (described below). The radiation beam covers the $2.5 \mathrm{~cm}$ active length of the chamber to avoid partial-volume irradiation uncertainties. The chamber is mounted on a motorized linear translator perpendicular to the beam axis to allow for profile scans as well as onaxis transmission measurements. The radiation beam enters the chamber from its side because head-on irradiation used in previous studies ${ }^{43,46,53,60,76}$ introduces unnecessary radiation-induced cable leakage and makes the Monte Carlo-calculated transmission data more sensitive to the chamber details (particularly the stem). The buildup caps used are the PMMA and W-alloy caps discussed throughout Ch. 2. A third pure Al cap is used for specific tests (described below).

The setup is aligned to a laser beam. Overall, the setup dimensions and the alignment are known with sub-mm accuracy. Temperature is recorded at the locations of the monitor and Farmer chambers to ensure that there is no differential temperature effect between the two locations (e.g., due to heat convection from the target). Pressure and humidity are also monitored. The experiment is automated and computer controlled using NRC Labview software, including the movement of the attenuator rack and the data acquisition from the three chambers (monitor, field and Farmer) and from other sensors. Each component is individually commissioned at the beginning of the experiment. The automated measurements are monitored with a CCTV system. Fig. 3.3 shows a view of the full measurement setup. 

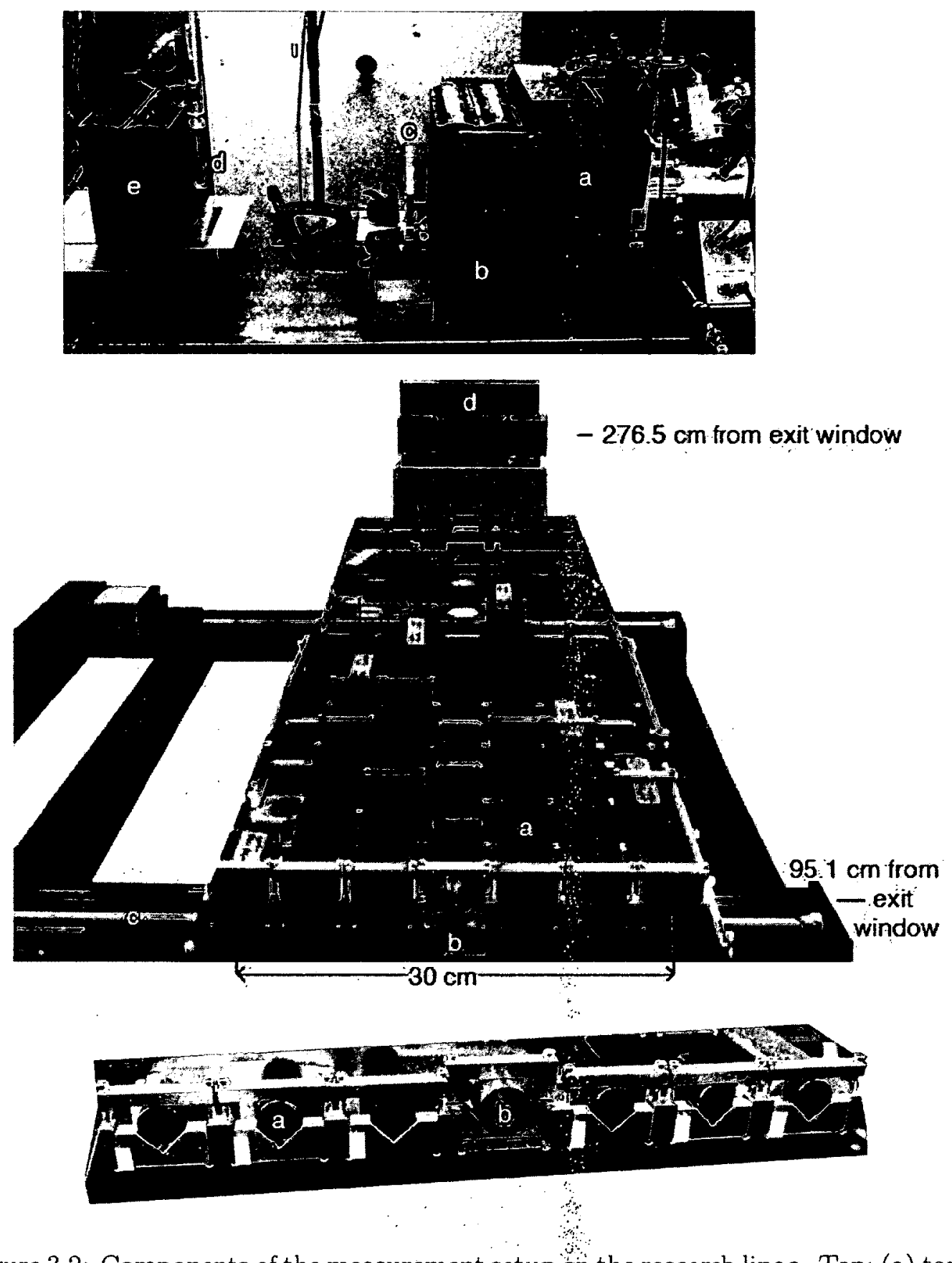

Figure 3.2: Components of the measurement setup on the research linac. Top: (a) target shielding, (b) first collimator, (c) monitor chamber, (d) field chamber, and, (e) second collimator. Middle: (a) graphite bars, (b) copperbenchmark rod (permanently fixed on the assembly), (c) Velmex bislide that enabling rack movement in the left-right direction, and, (d) third collimator. Bottom: (a) lead attenuator rods, and, (b) copper benchmark rod. Partly from Paper III. 


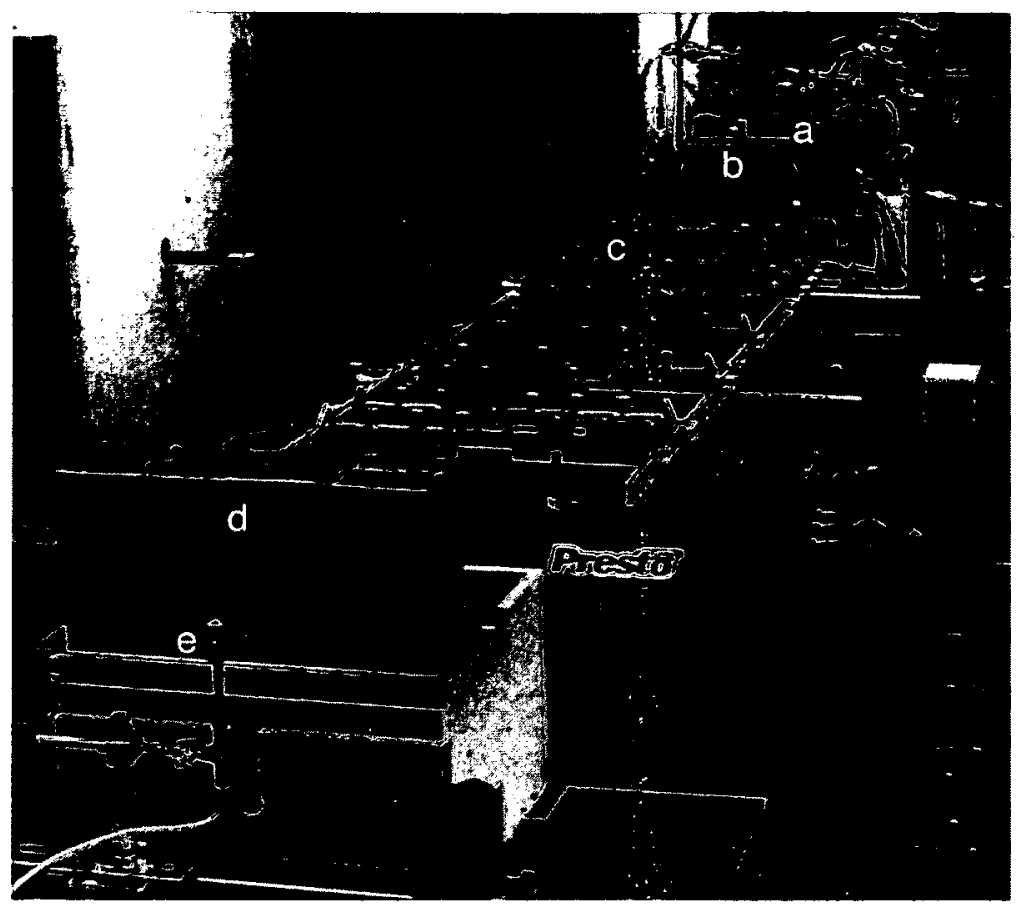

Figure 3.3: A view of the full measurement setup on the research linac. (a) target shielding, (b) second collimator, (c) graphite atteriuator assembly for the largest MV beam (30 MV), (d) third collimator, and, (e) A19 chamber with the W-alloy cap on a linear translator.

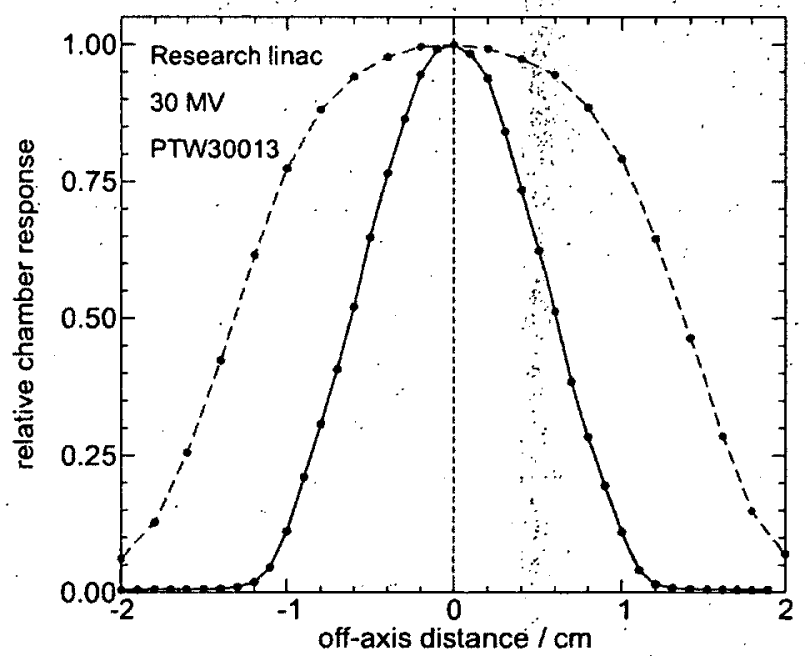

Figure 3.4: Horizontal beam profiles in the absence of an attenuator for the $30 \mathrm{MV}$ beam of the research linac. Solid line: past the first two collimators at the location of the upstream surface of the attenuator $(95.1 \mathrm{~cm}$ from the linac exit window). Dashed line: past the third collimator at the location of acquisition of the transmission signal (298.8 $\mathrm{cm}$ from the linac exit window). From Paper III. 


\subsection{Data acquisition and experimental corrections}

Equilibration: Irradiation during the initial daily setup reduces linac warmup effects and Farmer chamber settling effects. ${ }^{77}$ Settling/warmup effects for the electrometers are avoided by keeping them always ON and biased."

Steering: The extended dimensions of the setup and the high degree of collimation amplify small steering issues. Therefore, a PTW Starcheck 2D ion-chamber array ( $3 \mathrm{~mm}$ resolution) with a 2.5 -cm-thick PMMA buildup plate is periodically placed in front of the third collimator to check the steering. If necessary, the beam is manually steered until its peak aligns with the laser. Farmer chamber scans behind the third collimator are used to confirm profile symmetry, typically within $0.3 \mathrm{~mm}$ (re-steering if necessary). Fig. 3.4 shows an example of the measured beam profiles.

Typical measurement protocol and parameters: The five attenuator lengths for each attenuator/cap combination are placed on the translation system in random order to reduce bias from slow drifts in the beam direction. One data point is acquired per attenuator length, plus one data point with no attenuator and one with the $\mathrm{Cu}$ rod for a total of seven points. This process is repeated $4-8$ times to characterize short-term repeatability. Measurements are repeated with the Farmer chamber polarity reversed (discussed below). To characterize long-term repeatability, which assesses the stability of the energy of the electron beam, measurements for different beams and targets were repeated intermittently over 15 months. The $15 \mathrm{MV}$ beam has the largest number of long-term repeats (up to five long-term repeats per target per polarity over a year). Several of the sets measured with the PMMA cap are repeated with the Al cap, and a few of the sets measured with the A19 chamber are repeated with the PTW30013 chamber. These additional data are used to test the relative detector response calculations with EGSnrc, and to test the detector-independence of the unfolded spectra.

\subsection{DATA ACQUISITION AND EXPERIMENTAL CORRECTIONS}


Charge integration times varied from $5 \mathrm{~s}$ for the air signal to $60 \mathrm{~s}$ for the signal with the longest attenuators, leading to data acquisition time of $\leq 30 \mathrm{~min}$ per attenuator rack per polarity. The chosen integration times are short enough to allow for short-term beam instabilities to be identified by the field-to-monitor ratio and eliminated. Linearity of the signal with integration time was established. The uncertainty component for repeatability is not reduced with further increase in integration times.

The monitor chamber currents are $\sim 5$ to $20 \mathrm{nA}$ going from 10 to $30 \mathrm{MV}$, while the field chamber currents are $\sim 50$ times smaller. The Farmer chamber currents with the W-alloy cap and with no attenuator present are $\sim 50$ to $250 \mathrm{pA}$ going from 10 to $30 \mathrm{MV}$. These currents correspond to a dose rate to water of $\sim 15$ to $80 \mathrm{cGy} / \mathrm{min}$ if the bare chamber were in a water phantom. With the longest attenuators, the Farmer chamber currents are $\sim 60$ times lower than the currents with no attenuators. When the PMMA cap is used, the currents are roughly $60 \%$ of those with the W-alloy cap. Attempts to increase the beam currents for lower-MV beams to offset the lower bremsstrahlung yield were limited by poorer beam control and excessive target heating.

Data normalization and drift corrections: For a given attenuator rack, the temporal variation of the field-to-monitor chamber ratio exhibits one of four patterns. (a) A sharp change in the ratio, indicating a large temporary drift in the beam direction. The data during this unstable period are excluded during averaging. An example is shown by the solid line in Fig. $3.5 \mathrm{a}$ where a sudden $1 \%$ drop in the ratio is seen around minute 4. This emphasizes the importance of reasonably-short charge integration times. No universal threshold for data rejection is applied because the threshold depends on the overall temporal behavior of the field-to-monitor ratio. (b) A mostly-smooth change in the ratio (Fig. 3.5b), which indicates a slow drift in the beam direction that affects the field chamber signal but is not recognized by the monitor chamber due to its large sensitive volume. In this case, the Cu-to-monitor ratio is used to correct the Farmer-to-

\subsection{DATA ACQUISITION AND EXPERIMENTAL CORRECTIONS}




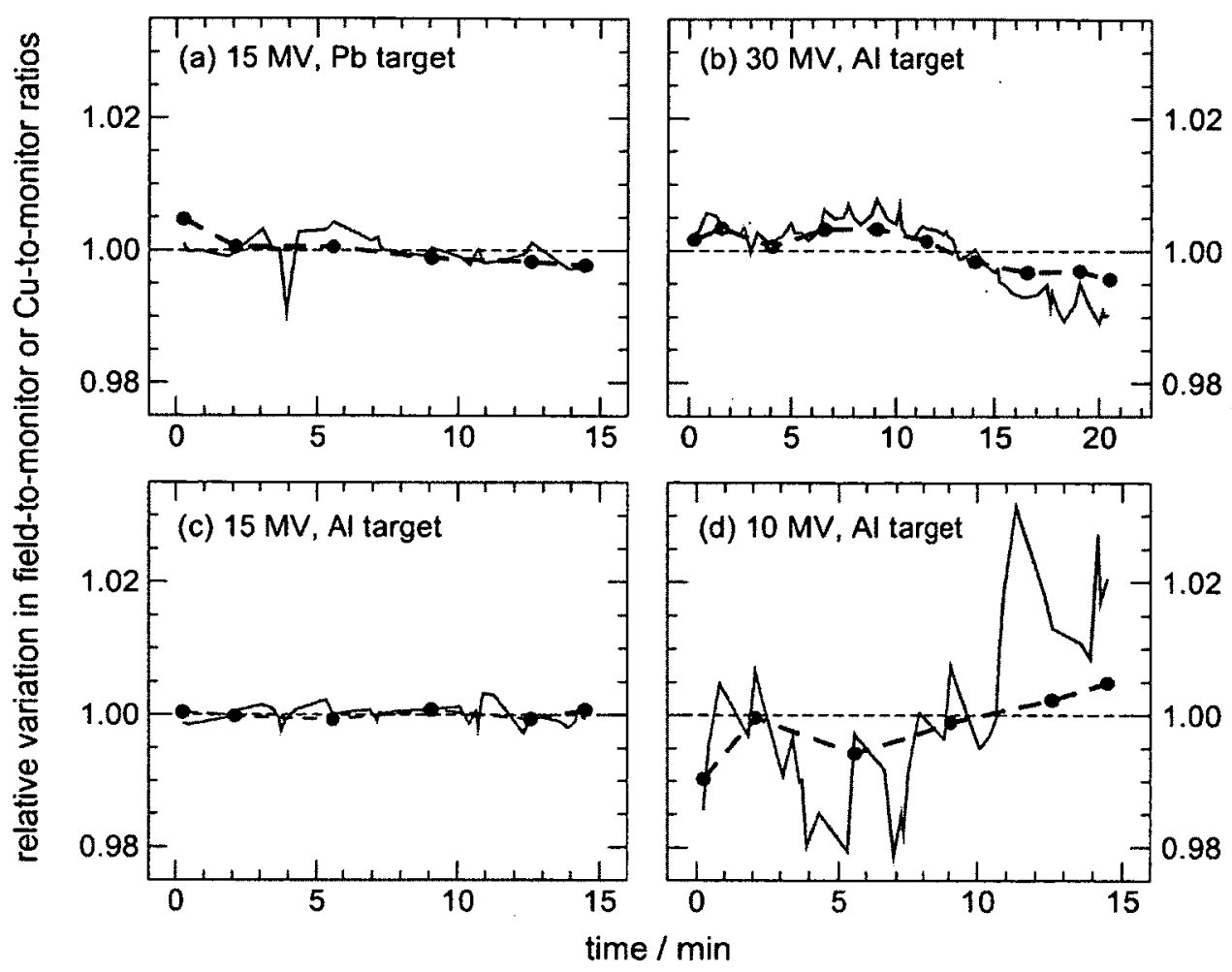

Figure 3.5: For the research linac beams, different temporal patterns of the fieldto-monitor ratio (solid lines with no symbols) and the Cu-to-monitor ratio (dashed lines with filled circles). The $\mathrm{Cu}$-to-monitor ratio is used for data rejection and drift correction. Note the different scales of the ordinates. From Paper III.

monitor signal for these second-order drifts. (c) Small random changes in the field-tomonitor ratio (Fig. 3.5c), which is the ideal situation since it indicates negligible drifts in the beam direction. In such a case the Cu-to-monitor correction is neither useful nor needed. (d) A slow change in the field-to-monitor ratio superposed on large fluctuations (Fig. 3.5d). In this case, the correction using the Cu-to-monitor signal accounts for part of the drift, and the rest of the fluctuations increase the measurement uncertainty. The $10 \mathrm{MV}$ beam of Fig. 3.5d was particularly noisier than other beams, which is found to be related to beam instability issues rather than signal-to-noise issues, and thus cannot be addressed by increasing the dose rate or the charge integration times. Overall, the magnitude of the correction for the drifts in the beam direction using the Cu-to-monitor ratio is $\leq 2 \%$.

\subsection{DATA ACQUISITION AND EXPERIMENTAL CORRECTIONS}


Leakage: Leakage is the signal in the absence of the radiation beam for the combined system of the Farmer chamber + extension cable + electrometer. In an initial setup, leakage from a $50 \mathrm{~m}$ cable made $\sim 50 \%$ of the total leakage $(1.3 \%$ for the smallest signals). Therefore the electrometer was moved inside the linac room, shielded, and connected to the chamber using a short cable to reduce the leakage to sub-fA $\pm 1 \mathrm{fA}$. The improved leakage uncertainty on the smallest transmission signals is $\leq 0.3 \%$.

Polarity effects: Strictly speaking, the polarity correction in this study, $P_{p o l}$, is an 'apparent relative' correction: 'apparent' because it is assumed to include all extracameral effects and radiation-induced leakage, and 'relative' because it is determined for the Farmer chamber signals after normalization to the respective monitor signals and after the drift correction by the $\mathrm{Cu}$-to-monitor ratio. The assumption made is that the causes of polarity cancel out when the absolute signals with the two opposite polarities are averaged. For all transmission data in this study, measurements are made at both polarities for the Farmer chamber $( \pm 300 \mathrm{~V})$. A five-minute waiting period is applied after polarity reversal to ensure that the chamber reached its equilibrium, because polarity cycling induces the worst settling behavior ${ }^{77}$ The good shielding in front of the stem of the Farmer chamber suggests that most of the correction is due to cable effects, rather than stem effects.

Fig. 3.6 shows examples of the measured $P_{p o l}$, defined as in the AAPM TG51 proto$\mathrm{col},{ }^{4}$ with the negative signal as the reference. The value of $P_{p o t}$ is typically unity within $2.5 \%$, but it can be as large as $6 \%$ for the smallest signals. The increase in $P_{\text {pol }}$ as the transmission signal decreases is because the extra-cameral effects are independent of the main signal size, and thus make a larger fraction of the smaller signals. The air signals with no attenuator (i.e., a transmission of unity) are the closest to typical clinical dose rates. For those signals, $P_{\text {pol }}$ values are consistent with TG51 recommendations ${ }^{4}$ (i.e. unity within $0.3 \%$ ). For some beams (e.g., the $20 \mathrm{MV}$ beam in Fig. 3.6), $P_{\text {pol }}$ depends on

\subsection{DATA ACQUISITION AND EXPERIMENTAL CORRECTIONS}




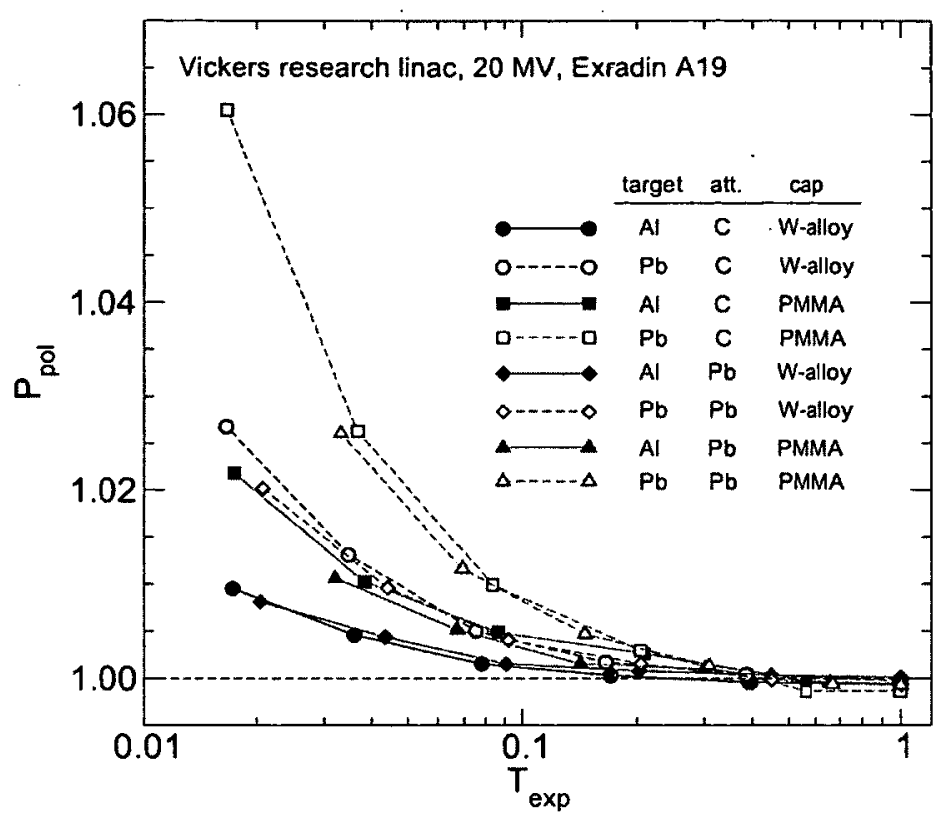

Figure 3.6: For the $20 \mathrm{MV}$ beam of the research linac, the polarity correction factor, $P_{p o l}$, versus the experimental transmission signals, $T_{\text {exp }}$. Not all MV beams require such a large correction. From Paper III.

the bremsstrahlung target material. However, no clear mechanism is found to explain such clear target dependence, particularly that it is not the case for all MV beams. The value of $P_{p o l}$ is larger for the PMMA cap compared with the W-alloy cap, which could be caused by the smaller signal with the PMMA cap and/or by more scatter from the cap into the cables. There is only a very subtle increase in $P_{p o l}$ for the $\mathrm{C}$ attenuators compared with the $\mathrm{Pb}$. For the PTW30013 chamber, the magnitudes of $P_{\text {pol }}$ values and their variation with transmission are different from those for the A19 chamber (not shown). The observations above collectively underline that the polarity correction is non-negligible and that it is sensitive to minor detector and setup details and should thus always be measured for the exact setup used.

Ion recombination: $P_{i o n}$ is investigated because charge collection efficiency varies with dose rate, which changes by a factor of $\sim 60$ in a transmission curve. The correction is determined using the approach of McEwen. ${ }^{78}$ For the most extreme case (30 MV

\subsection{DATA ACQUISITION AND EXPERIMENTAL CORRECTIONS}


beam and a W-alloy cap), $P_{i o n}$ varies from $0.18 \%$ to $0.07 \%$ going from the largest to the smallest transmission signals. Therefore its maximum differential effect is only $0.11 \%$.

Room scatter: Room scatter is the corrected Farmer chamber signal when the radiation beam is $\mathrm{ON}$ but completely blocked. Unlike the polarity effect, room scatter contributes to the cavity ionization, rather than to the cable signal. It is experimentally determined using the shadow-cone technique whereby a $40-\mathrm{cm}-$ long $\mathrm{Pb}$ rod is placed at $95.1 \mathrm{~cm}$ from the exit window to attenuate the photon beam by many orders of magnitude, and the Farmer chamber signal is acquired at the far end. Measurements are made for different MV beams at both polarities and with the A19 and PTW30013 chambers. The signals are corrected by $P_{p o l}$ and $P_{i o n}$. Room scatter is found to be $0.8 \%$ of the smallest signal for the $30 \mathrm{MV}$ beam, and much smaller for lower-MV beams. Its magnitude is largely chamber-independent.

Apparatus scatter: The effect of the apparatus components that are not part of the Monte Carlo model is investigated here. These components are the side attenuators, the table holding the setup, and the Al support rack. For side attenuators, comparing the measured signals for a given bar with and without various side attenuator configurations show that their contribution is negligible. The permanent $\mathrm{Cu}$ rod experiences different side attenuators in different racks, and its signal remains constant within repeatability. For table scatter, the effect is investigated by adding a large Al plate on top of the attenuator rack to mirror the table effect, and no signal increase is observed. For the Al support rack, transmission signals measured with and without the rack are the same within repeatability. EGSnrc sensitivity studies indicate that the combined scatter from the three components is $\leq 0.01 \%$ of the smallest transmission values.

Based on the details above, the following data processing sequence is applied for each MV/target/attenuator/detector combination. (a) The data acquired during shortterm beam instabilities are identified and excluded. (b) The Farmer signals are nor- 
malized to their respective monitor chamber signals. (c) Drift corrections are applied using the $\mathrm{Cu}$-to-monitor ratio. (d) Short-term repeats for a given polarity are averaged. (e) Polarity and ion recombination corrections are applied. (f) The corrected room scatter signal is subtracted from the corrected Farmer chamber signals. (g) Long-term repeats of the corrected Farmer chamber signals are averaged. (h) The averaged data are normalized to the air signal to obtain a fully-corrected transmission curve.

\subsection{Experimental uncertainty budget}

The experimental uncertainty budget, derived according to The ISO Guide on Uncertainty in Measurement, ${ }^{79}$ is given in Table 3.2.

Short-term beam drifts, $P_{p o l}$ and leakage: The conservative approach adopted is to assume that the three components are independent and can be added in quadrature. The first two components are evaluated by calculating the statistical uncertainty of shortterm repeats for polarity-corrected signals, then assuming that $\frac{1}{\sqrt{2}}$ of that uncertainty is due to beam direction drifts that have not been fully accounted for, and the other $\frac{1}{\sqrt{2}}$ is due to the uncertainty on polarity correction. For the $10 \mathrm{MV}$ beam, the drift and polarity uncertainties are twice those for other beams, and they are less dependent on the signal size. This is reflective of the bearn instability issues discussed earlier for that beam.

Long-term repeatability: This component characterizes the stability of the electron beam energy, and it indicates changes on top of the short-term ones. The air-to-Cu signal was monitored for the $15 \mathrm{MV}$ beam for a year and was found to be constant within $0.1 \%$. This is another important use of the $\mathrm{Cu}$ signal which confirms that the incident electron energy has not changed. The klystron had to be replaced during the course of the measurements, and excellent long-term repeatability was still achieved, which provides confidence in the values assigned to this component.

\subsection{EXPERIMENTAL UNCERTAINTY BUDGET}


Table 3.2: For the research linac measurements, the uncertainty budget of the experimental transmission signals, $T_{\text {exp }}$. Values are given for one relative standard uncertainty, $u$. Some components are beam-specific, thus the $10 \mathrm{MV}$ beam is presented separately. The leakage component is estimated by statistical means (Type A), while all others are estimated by non-statistical means (Type B). The total uncertainty is obtained by adding its components in quadrature. The total uncertainty is given without and with the components from the electron beam because those components are not known for typical clinical beams and they are only included for the research linac beams because the data are used as a primary benchmark. From Paper III.

\begin{tabular}{|c|c|c|c|c|}
\hline \multirow{3}{*}{ Uncertainty component } & \multicolumn{4}{|c|}{$u$ in per cent for $T_{e x p}: \sim 1 \rightarrow \sim 0.017$} \\
\hline & \multicolumn{2}{|c|}{$10 \mathrm{MV}$} & \multicolumn{2}{|c|}{$15,20,30 \mathrm{MV}$} \\
\hline & $\mathrm{C}$ att. & $\mathrm{Pb}$ att. & C att. & $\mathrm{Pb}$ att. \\
\hline \multicolumn{5}{|l|}{ Linac and detection system } \\
\hline $\begin{array}{l}\text { Short-term beam drifts } \\
P_{\text {pol }} \\
\text { Leakage } \\
\text { Long-term repeatability } \\
P_{\text {ion }} \\
\text { Monitor chamber stability } \\
\text { Electrometer nonlinearity }\end{array}$ & $\begin{array}{c}0.2 \rightarrow 0.25 \\
0.2 \rightarrow 0.25 \\
0.003 \rightarrow 0.3 \\
0.2 \rightarrow 0.2 \\
0.03 \\
0.1 \\
0.05\end{array}$ & $\begin{array}{c}0.2 \rightarrow 0.25 \\
0.2 \rightarrow 0.25 \\
0.003 \rightarrow 0.3 \\
0.2 \rightarrow 0.2 \\
0.03 \\
0.1 \\
0.05\end{array}$ & $\begin{array}{c}0.07 \rightarrow 0.15 \\
0.07 \rightarrow 0.15 \\
0.001 \rightarrow 0.1 \\
0.1 \rightarrow 0.15 \\
0.03 \\
0.1 \\
0.05\end{array}$ & $\begin{array}{c}0.07 \rightarrow 0.15 \\
0.07 \rightarrow 0.15 \\
0.001 \rightarrow 0.1 \\
0.1 \rightarrow 0.15 \\
0.03 \\
0.1 \\
0.05\end{array}$ \\
\hline \multicolumn{5}{|l|}{ Attenuators } \\
\hline $\begin{array}{l}\text { Mass thickness } \\
\text { Density non-uniformity } \\
\text { Impurities }\end{array}$ & $\begin{array}{c}0.1 \\
0.1 \\
0.05\end{array}$ & $\begin{array}{c}0.15 \\
0.0 \\
0.02\end{array}$ & $\begin{array}{c}0.1 \\
0.1 \\
0.05\end{array}$ & $\begin{array}{c}0.15 \\
0.0 \\
0.02\end{array}$ \\
\hline \multicolumn{5}{|l|}{ Incident electron beam } \\
\hline $\begin{array}{l}\text { Mean energy } \\
\text { Radial spread } \\
\text { Divergence }\end{array}$ & $\begin{array}{c}0.15 \rightarrow 0.67 \\
0.15 \\
0.1\end{array}$ & $\begin{array}{c}\sim 0.05 \\
0.15 \\
0.1\end{array}$ & $\begin{array}{c}0.1 \rightarrow 0.5 \\
0.15 \\
0.1\end{array}$ & $\begin{array}{c}0.01 \rightarrow 0.2 \\
0.15 \\
0.1\end{array}$ \\
\hline \multicolumn{5}{|l|}{ Total uncertainty } \\
\hline $\begin{array}{l}\text { Without } u \text { of } \mathrm{e}^{-} \text {beam } \\
\text { With } u \text { of } \mathrm{e}^{-} \text {beam }\end{array}$ & $\begin{array}{l}0.41 \rightarrow 0.55 \\
0.47 \rightarrow 0.88\end{array}$ & $\begin{array}{l}0.41 \rightarrow 0.55 \\
0.45 \stackrel{\rightarrow}{\rightarrow} 0.58\end{array}$ & $\begin{array}{l}0.26 \rightarrow 0.35 \\
0.33 \rightarrow 0.64\end{array}$ & $\begin{array}{l}0.26 \rightarrow 0.35 \\
0.31 \rightarrow 0.44\end{array}$ \\
\hline
\end{tabular}

${ }^{a}$ The corresponding component for the Farmer chamber is negligible. ${ }^{78}$ 
Attenuator mass thickness: For C, when transmission data are acquired for different bar combinations that add up to the same physical length, variations of up to $1.2 \%$ are observed. When EGSnrc calculations for these bar combinations are performed using their individual mass thicknesses, the relative variations in the EGSnrc transmission data exquisitely matched the experimental observations within $0.1 \%$ above repeatability. This indicates that the individual mass thicknesses should be used in the Monte Carlo calculations. The $0.1 \%$ level of agreement just mentioned is taken as the uncertainty component from mass thickness variations that are unaccounted for. For $\mathrm{Pb}$, the uncertainty comes from using the average density due to minor rod irregularities.

Attenuator density non-uniformity: For $\mathrm{C}$, its manufacturing method (isomolding) and its very fine grain size $(10 \mu \mathrm{m})$ suggest excellent density uniformity. CT scans of the bars did not indicate any bores or patterns, and suggested a $0.5 \%$ estimate of density nonuniformity (a better estimate was precluded by imaging and reconstruction artifacts). To supplement the CT results, transmission measurements are compared with and without the bars rotated in the orthogonal and longitudinal directions. This allows the radiation beam cone to sample different portions of the bars. Variations at the $0.1 \%$ level above repeatability are observed, which is taken as the uncertainty component for density non-uniformity. For $\mathrm{Pb}$, this component is negligible.

Attenuator impurities: The effect of reasonable variations in impurities is calculated deterministically (Eq. 1.1, p. 8) for a few spectra. The uncertainty is larger for C than it is for $\mathrm{Pb}$ because the ash content is 'assumed', rather than 'supplied'.

Incident electron beam parameters: The uncertainty components from the incident electron beam are investigated because this is a primary benchmark. The total uncertainty is given without and with these components because they are not known for typical clinical beams and, thus, would not be part of an uncertainty budget of transmission measurements on a typical clinical linac. For the mean energy, EGSnrc calculations are

\subsection{EXPERIMENTAL UNCERTAINTY BUDGET}


performed with mean energies both at the actual bending magnet values and at values one standard deviation ( $0.4 \%)$ larger. The effect on the calculated transmission data is much larger with the $\mathrm{C}$ attenuator than it is with $\mathrm{Pb}$ (the reason is discussed in $\S 4.5$ ), and it is the largest component in the uncertainty budget for C. The uncertainty component from the focal spot size is estimated from the difference in EGSnrc calculations when the FWHM of the radial spread is changed from 1 to $2 . \mathrm{mm}^{69}$

Positioning and alignment: To investigate the uncertainty component related to positioning, transmission signals are measured with $\mathrm{C}$ and $\mathrm{Pb}$ attenuators shifted continuously in sub-mm intervals. Given the lateral dimensions and the physical extent of the attenuators of both materials, experimental transmission signals did not change for shifts up to 5 and $2 \mathrm{~mm}$ for $\mathrm{C}$ and $\mathrm{Pb}$, respectively. Offsetting the Farmer chamber position in the vertical direction within $\pm 1.5 \mathrm{~mm}$ did not show any differential effect, both experimentally and in EGSnrc simulations. Therefore attenuator and detector positioning uncertainties are assumed negligible. For the uncertainty component due to misalignment, transmission measurements after repeated re-installation of the three collimators show that this component is negligible.

Other influence quantities: (a) The potential charge storage in the large insulating PMMA $\operatorname{cap}^{80}$ is investigated experimentally by comparing repeats at the end of a day or a week of heavy irradiation against those with fresh irradiation. The effect is found to be negligible. (b) Since the two buildup caps used have different physical dimensions, the difference in the spectra seen by the two caps is investigated using EGSnrc and found to be negligible. (c) Since the clinically-useful primary spectrum is the one at the machine isocenter rather than the one at the chamber location at $\sim 3 \mathrm{~m}$, the primary photons at the isocenter that do not reach the detector because of their larger divergence angle are investigated using EGSnrc and their effect on the spectrum is found to be negligible.

\subsection{EXPERIMENTAL UNCERTAINTY BUDGET}




\subsection{Clinical linac measurements}

Transmission measurements are performed on-axis on an Elekta Precise for 6, 10 and $25 \mathrm{MV}$ beams. The apparatus (Fig. 3.7) and measurement protocol are the same as those for the research linac beams, except for the specific details below.

Experimental setup: The first stage of collimation is achieved using the linac internal jaws to create a $3 \times 3(\mathrm{~cm})^{2}$ field at $100 \mathrm{~cm}$. A second $\mathrm{Pb}$ collimator of $10.2-\mathrm{cm}$ thickness and a non-diverging $0.77-\mathrm{cm}$-diameter opening is mounted on a custom 'shadow tray' at $60 \mathrm{~cm}$ from the upstream surface of the bremsstrahlung target. An in-house external monitor chamber is mounted between the jaws and the second collimator. Its sensitive volume is a cylinder of diameter $11.5 \mathrm{~cm}$ and thickness $2 \mathrm{~mm}$. The chamber is used for output normalization instead of the linac built-in multi-element chamber to avoid potential uncertainties from the complex feedback mechanisms of the latter. Unlike the measurements on the research linac, the small field created by the jaws precludes the use of a field chamber between the two collimators to monitor drifts in the beam

direction. However, the signal from a short $\mathrm{Cu}$ rod is acquired, and the ' $\mathrm{Cu}$-to-monitor' ratio is used to correct for linac drifts as done in the research linac measurements. The upstream surface of the attenuators is placed at the plane of the machine isocenter at $100 \mathrm{~cm}$. For the 6,10 , and $25 \mathrm{MV}$ beams, respectively, the maximum attenuator lengths are 64,83 and $116 \mathrm{~cm}$ for $\mathrm{C}$, and $7.5,7.5$, and $7.0 \mathrm{~cm}$ for $\mathrm{Pb}$. The corresponding smallest transmission signals are $\sim 2 \%$. For the $6 \mathrm{MV}$ beam, additional measurements are performed with $\mathrm{Cu}$ attenuators (maximum length $11 \mathrm{~cm}$ ). The $\mathrm{Cu}$ data are used to test the attenuator-independence of the unfolded spectra by comparing the spectra unfolded using $\mathrm{C}+\mathrm{Cu}$ data against those using $\mathrm{C}+\mathrm{Pb}$ data. Past the attenuators, the details are the same as those for the research linac measurements. The setup is aligned at the $1 \mathrm{~mm}$ level at $\sim 3 \mathrm{~m}$ using the light field and two orthogonal telescopes. Compared 
with the soft flattening-filter-free beams of the research linac, the clinical beams have flatter profiles at the Farmer chamber location, which further reduces the effect of chamber positioning uncertainties. Measurements are performed using a horizontal beam position to reduce floor backscatter into the chamber and cables.

Data acquisition: The measured Farmer chamber currents with the W-alloy cap and with no attenuator present are $\sim 150$ to $250 \mathrm{pA}$ going from 6 to $25 \mathrm{MV}$. These currents correspond to a dose rate to water of $\sim 45$ to $75 \mathrm{cGy} / \mathrm{min}$ if the bare chamber were in a water phantom. With the longest attenuators, the Farmer chamber currents are $\sim 50$ times lower. Unlike the research linac, which has minimal feedback, the continuous feedback in the clinical linac causes larger fluctuations in the signals with short charge collection time ( $5 \mathrm{~s}$ for the signals with no attenuators). To maintain a $0.1 \%$ standard deviation on the mean for short-term repeatability, the number of repeat measurements is increased threefold compared to the research linac measurements.

Experimental corrections: The correction for output fluctuations using the external monitor chamber signal is $\leq 1 \%$, and the correction for short-term beam drifts using the 'Cu-to-monitor' ratio is $\leq 0.25 \%$. These corrections are smaller than their respective ones for the research linac beams $(\leq 3.5 \%$ and $\leq 2 \%)$ because the continuous feedback mechanisms in the clinical linac lead to a more stable output. The polarity correction, $P_{\text {pol }}$, is shown in Fig. 3.8. It is the largest correction and can be up to $6 \%$ for the smallest signals in the highest-MV beam. The larger $P_{p o l}$ for the $25 \mathrm{MV}$ beam can be explained by the larger scatter signals into the chamber cables, which can be due to increased head leakage, increased leakage through the collimators, and larger fraction of the beam penetrating through the detector - all of which lead to more radiation in the room. Similar to the research beams, $P_{p o l}$ is larger for the PMMA cap compared with the W-alloy cap, and marginally larger for the $\mathrm{C}$ attenuators compared with the 


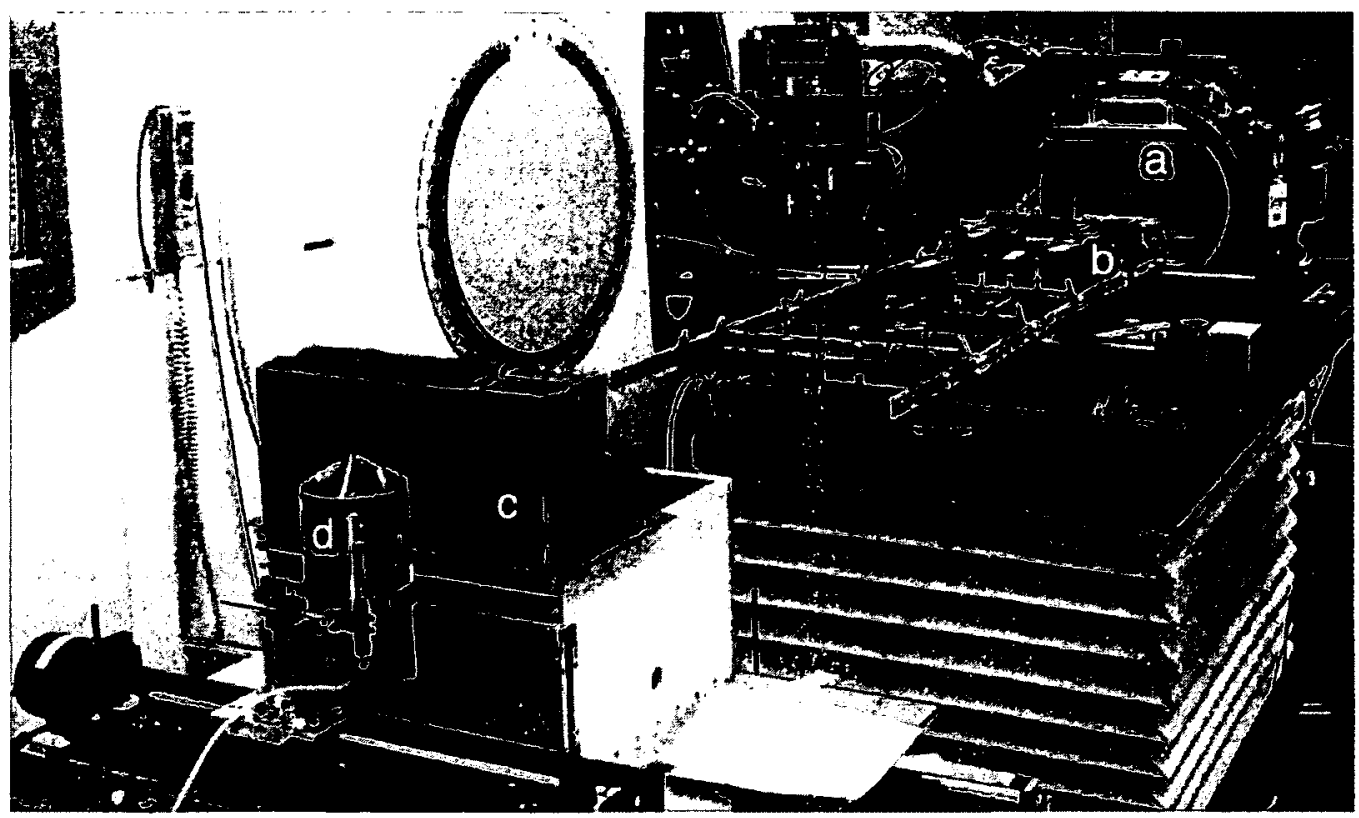

Figure 3.7: A view of the full measurement setup on the clinical linac. (a) Al shadow tray with lead collimation mounted on it, (b) graphite attenuator assembly for the lowest MV beam (6 MV), (c) third collimator, and, (d) A19 chamber with the PMMA cap on a linear translator.

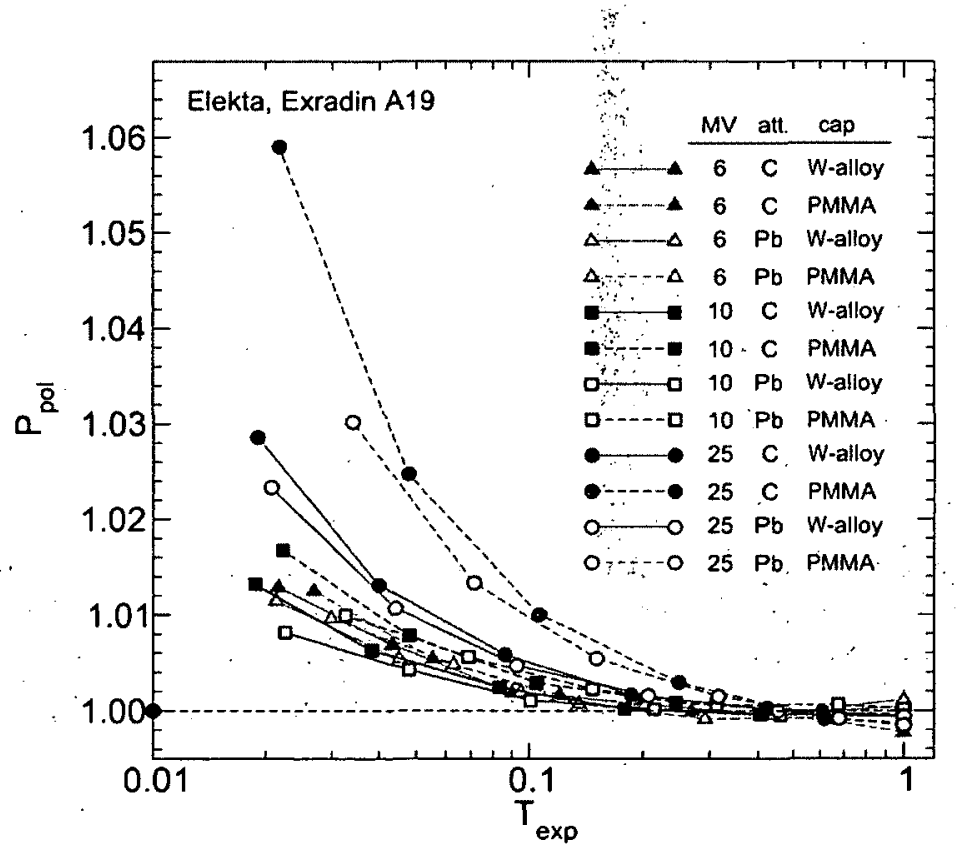

Figure 3.8: For the clinical linac beams, the polarity correction factor, $P_{\text {pol }}$, versus the experimental transmission signals, $T_{\text {exp }}$. From Paper IV. 
$\mathrm{Pb}$ attenuators. Corrections for ion recombination and room scatter are small and very similar to those for the research beams.

Uncertainty budget: An uncertainty budget for the fully-corrected transmission signals is constructed similar to that done for the research beams (Table 3.2, p.55). The two main differences for the clinical beams are: (a) the uncertainty component due to short-term repeatability is smaller, and, (b) the uncertainty components on the incident electron bearn parameters are not included because they are unknown and because the clinical linac is not used as the primary benchmark. The total standard uncertainty on the smallest signals is $0.35 \%$.

\subsection{Summary}

In this chapter, methods are presented for automated transmission measurements, down to $\sim 1.7 \%$, on the research linac (eight beams) and on a typical clinical linac (three beams). Measurements are made using the attenuator/detector configuration that gives the best energy sensitivity (C4 in $\S 2.5$ ). Experimental corrections are applied for drifts in the beam direction (2\%), polarity (2.5\% typical maximum, $6 \%$ extreme), ion recombination $(0.2 \%)$, leakage $(0.3 \%)$ and room scatter $(0.8 \%)$ - the values in parentheses are the largest corrections applied. Based on a detailed uncertainty budget, the uncertainty on the smallest transmission signals is $\sim 0.4 \%$. The dominant potential contributors to measurement uncertainties are beam drifts, polarity effects, leakage and attenuator mass thickness. Ignoring the investigated experimental influence quantities can collectively introduce errors of more than $10 \%$ in the measured signals. The measured data presented in this chapter are used for benchmarking EGSnrc (Ch.4) and for unfolding spectra and incident electron energies (Ch. 5). 


\section{Chapter 4}

\section{A sensitive EGSnrc benchmark}

Transmission measurements are a particularly sensitive primary benchmark of Monte Carlo codes (compared with, e.g., depth-dose measurements) because the extreme attenuation, the extreme collimation, and the lack of volume scatter collectively amplify small effects that would otherwise be averaged out. No previous studies used megavoltage transmission measurements to benchmark a Monte Carlo code because for clinical linacs the electron parameters are not known accurately, and the head geometry is complex. In this chapter, the measured transmission data from $\mathrm{Ch} .3$ for the research linac beams are used to benchmark EGSnrc since the electron parameters for this linac are independently known and the geometry is simple and accurately known. The benchmark includes relative sensitivity studies of transmission data ( $\$ 4.5)$, and direct comparisons of EGSnrc-calculated data against measurements ( $\$ 4.6)$. The benchmark is also used to validate the EGSnrc-calculated detector energy response from $\S 2.3$, and to identify the limiting factors that affect the accuracy of transmission calculations.

\subsection{Modelling the research linac setup}

BEAMnrc $^{37,38}$ is used to model the measurement setup on the research linac from the exit window to the downstream end of the third collimator using the details given in Ch. 3. The incident electron parameters are taken from $§ 3.1$. Based on the discus- 
sions in $\S 3.2$ and $\S 3.4$, the individual mass thicknesses of the $\mathrm{C}$ attenuators are used, while the average density and the physical lengths of the $\mathrm{Pb}$ attenuators are used. Attenuator impurities are modelled. The BEAMnrc simulation efficiency is improved using directional bremsstrahlung splitting (DBS) ${ }^{41}$ as well as variable photon splitting at the upstream face of the attenuator. The usercode cavity ${ }^{39}$ is used to model the Farmer chambers (Exradin A19 and PTW30013) from their blueprints, ${ }^{73}$ fitted with the W-alloy, PMMA or Al caps. BEAMnrc is used as a shared library input to cavity to eliminate the need for phase-space storage and for particle recycling. Doses to the cavity of the chamber are calculated to $\sim 0.15 \%$ statistical uncertainty.

The most accurate low- and high-energy physics options available in EGSnrc are used in all simulations. This has to be done because the extended dimensions $(\sim 3 \mathrm{~m})$, the extreme attenuation and the extreme collimation strongly amplify what would otherwise be small physics effects. For instance, Rayleigh scattering is commonly known to be relevant only for low energies. However, EGSnrc simulations for the $10 \mathrm{MV}$ beam (the lowest MV beam, with $1.5 \mathrm{MeV}$ mean photon energy) show that ignoring Rayleigh scattering with $\mathrm{Pb}$ attenuators leads to errors up to $2 \%$ in the smallest transmission data. Other second-order effects that are turned ON are incoherent scattering corrections (binding effects, radiative corrections and double Compton), electron-electron bremsstrahlung in the target, electron impact ionization, and explicit triplet production. The photon energy cutoff is $10 \mathrm{keV}$. The refined NIST XCOM cross sections from App. $C$ are used for all simulations.

\subsection{Bremsstrahlung angular sampling}

The EGSnrc-calculated transmission data are found to be particularly sensitive to the choice of the bremsstrahlung angular sampling option. EGSnrc offers two sampling options: KM and Simple - see $\S 1.5$ for a needed introduction. It is not immediately 
obvious which of the two options is more accurate because the underlying assumptions are not strictly satisfied for either of them. Fig. 4.1 shows the effect of the angular sampling options on the calculated transmission data. The effect is because, after sampling the energy of a bremsstrahlung photon, the different formulae used to sample the emission angle lead to slightly different spectra seen by the Farmer chamber due to the high degree of collimation. The effect generally increases with the atomic number of the target and can be up to $5 \%$. An important observation is that the absolute cavity doses (i.e., before normalization to the cavity dose with no attenuator) when using the two sampling options differ by up to $16 \%, 9 \%$ and $4 \%$ for the $\mathrm{Be}, \mathrm{Al}$ and $\mathrm{Pb}$ targets, respectively. A related observation is that in the previous $\mathrm{NaI}$ measurements of photon spectra on the same linac, the absolute EGSnrc yield calculations ${ }^{6}$ on the beam axis using the $\mathrm{KM}$ option were within the $5 \%$ experimental uncertainties of the $\mathrm{NaI}$ measurements. Combining these two observations, it can be concluded that for on-axis yields the KM option is more accurate. Therefore the KM option is used throughout this study. This worsens the simulation efficiency for the research linac setup by a factor of 2.6. A typical calculation, not optimized for efficiency, takes of the order of a few tens of hours on a single $3.6 \mathrm{GHz}$ CPU core. Finally, it is difficult to use only the comparison between the measured and calculated transmission data of this study to draw a conclusion regarding the accuracy of the KM and Simple options. This is because cross section uncertainties are amplified by the strong attenuation, which complicates the analysis.

\subsection{Modelling photonuclear attenuation}

In the context of deterministic calculations of transmission data using Eq. 1.1 (p. 8) during the unfolding, it was demonstrated in Fig. 2.8 (p.33) that photonuclear attenuation is important for accurate calculations. The same argument applies to transmission data

\subsection{MODELLING PHOTONUCLEAR ATTENUATION}




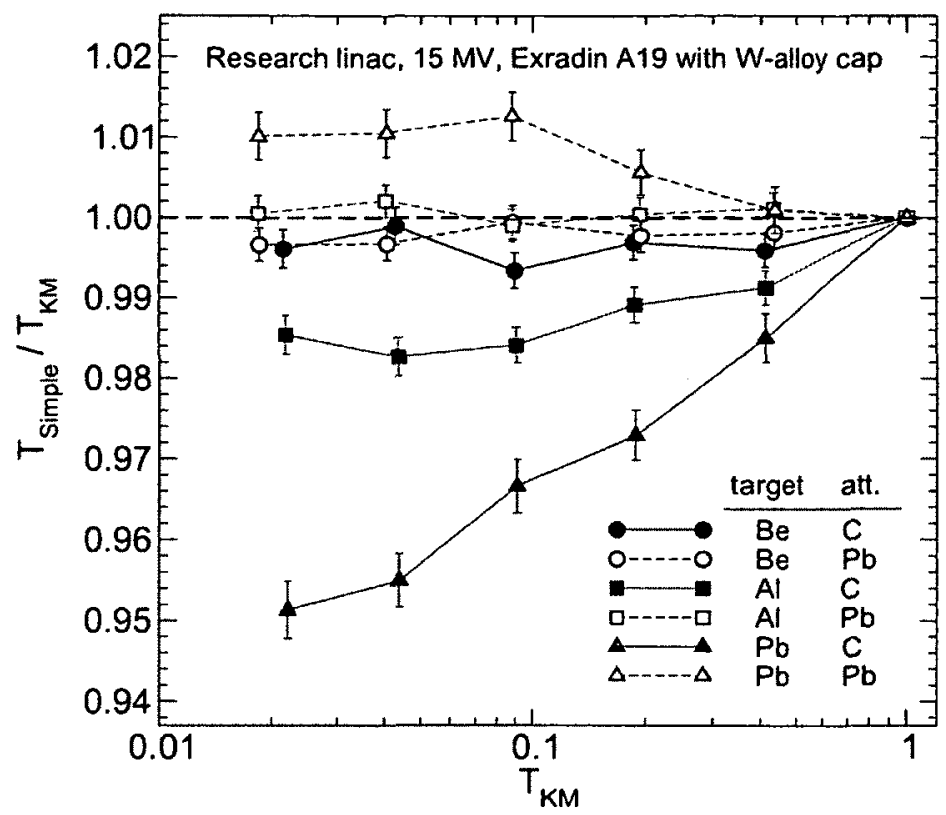

Figure 4.1: For the $15 \mathrm{MV}$ beam of the research linac, the effect of using the different bremsstrahlung angular sampling options offered in EGSnrC (KM and Simple) on the calculated transmission, $T$. The $15 \mathrm{MV}$ beam is typical of other beams. From Paper III.

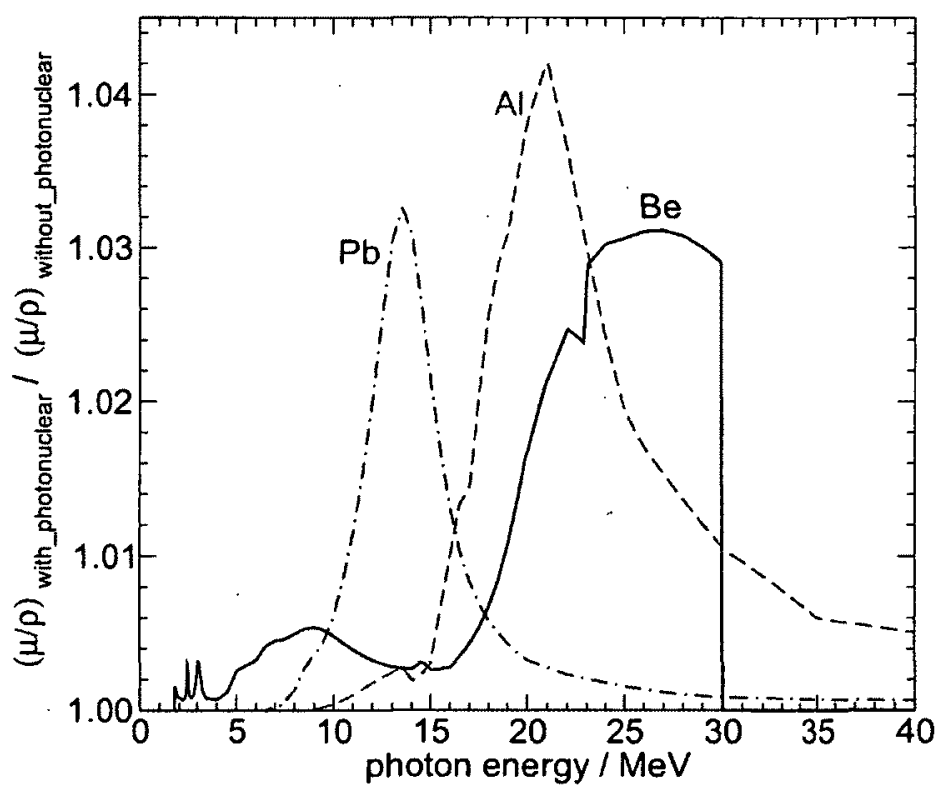

Figure 4.2: For the target materials of the research linac, fractional increase in the NIST XCOM total cross sections when the IAEA photonuclear data are included. From Paper V. 


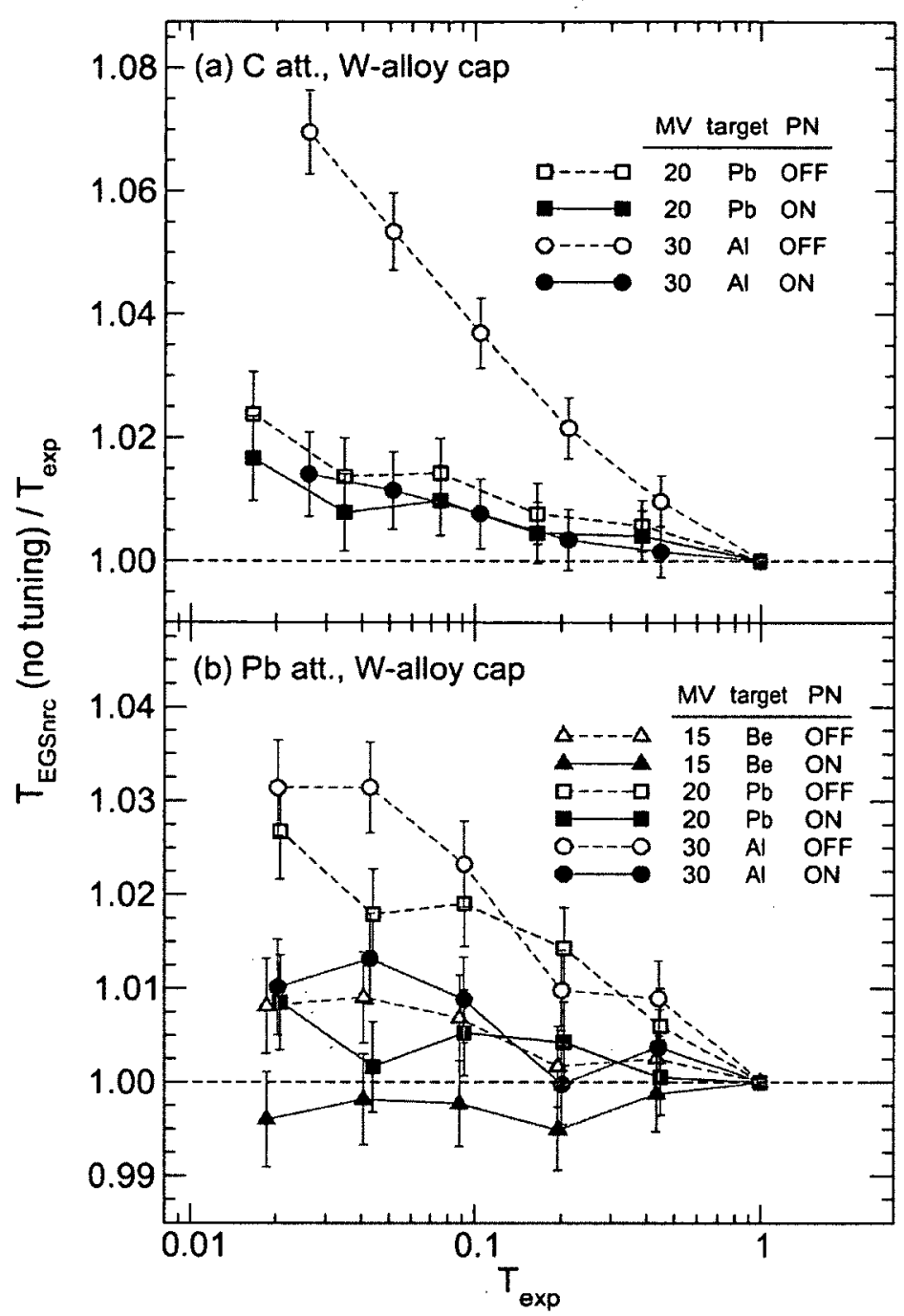

Figure 4.3: For the high-MV beams of the research linac, the figure shows the improvement in the agreement between EGSnrc-calculated transmission, $T_{E G S n r c}$, and those measured experimentally, $T_{\text {exp }}$, when the photonuclear effect (PN) is modelled in EGSnrc. The $1 \sigma$ uncertainty bars are obtained by adding in quadrature the totals of the uncertainty budgets for $T_{\exp }$ (Table 3.2, p. 55) and $T_{\text {EGSnrc }}$ (Table 4.1, p.68). Photon cross section uncertainties are not included. From Paper V. 
calculated by Monte Carlo. As part of this study, EGSnrc is upgraded to model photonuclear attenuation without modelling secondary particles. The implementation allows for turning photonuclear attenuation $\mathrm{ON}$ on all the geometric regions of a simulation, or on a region-by-region basis. A summary of this work is given in App. D. All EGSnrc calculations that model the experimental setup have photonuclear attenuation ON everywhere except in the detector.

The main photonuclear effect is from the attenuators because of their large mass thicknesses (their photonuclear cross sections are given in Fig. 2.7, p. 32). Moreover, the strong variation of photonuclear cross sections with energy for the targets (Fig. 4.2, p. 65) and for other minor materials in the path of the photon beam leads to small spectral differences, which leads to additional effects on the EGSnrc-calculated transmission data. Fig. 4.3 shows that for the high-MV beams, modelling photonuclear attenuations everywhere in the geometry significantly improves the agreement between measured and EGSnrc-calculated transmission data. For the $30 \mathrm{MV}$ beam with the longest graphite attenuator, the $5.6 \%$ photonuclear effect is $5.2 \%$ from the $\mathrm{C}$ attenuator and $0.4 \%$ from the Al target.

\subsection{Monte Carlo uncertainty budget}

The uncertainty budget for the EGSnrc-calculated transmission data is given in Table 4.1. The uncertainty from the detector energy response is deduced from the level of agreement between measurements and EGSnrc calculations for the relative Farmer chamber response ( $(4.5)$. The fundamental quantity $W / e$ is the amount of energy deposited in the air cavity per Coulomb of charge released, and it is assumed constant with electron energy. The upper bound estimate on the variation of $W / e$ from ${ }^{60} \mathrm{Co}$ to $25 \mathrm{MV}$ is $0.25 \%$ ( $68 \%$ confidence).$^{81}$ Applying this to the range of spectral variation versus transmission gives an uncertainty of $\sim 0.1 \%$. From the large effects shown

\subsection{MONTE CARLO UNCERTAINTY BUDGET}


Table 4.1: The uncertainty budget for the EGSnrc-calculated transmission data. Values are given for one relative standard uncertainty, $u$. The simulation statistical uncertainty is Type A and the other components are Type B. The total uncertainty is obtained by adding its components in quadrature. From Paper III.

\begin{tabular}{|l|c|}
\hline Uncertainty component & $u / \%$ \\
\hline Simulation statistical uncertainty & 0.15 \\
Detector energy response & 0.15 \\
EGSnrc wrt its own cross sections (Fano test) & 32 \\
$W / e$ variation with electron energy & 0.1 \\
Bremsstrahlung energy-angle distributions & not included \\
Cross section uncertainties & not included \\
& \\
Total & 0.25 \\
\hline
\end{tabular}

in $\S 4.2$ when using different bremsstrahlung angular sampling options, it can be extrapolated that the accuracy of even the more accurate energy-angle distribution will have an effect on the calculated transmission data, but it is beyond the scope of this study. The photon cross section uncertainties are discussed separately in Ch.6. Geometric uncertainties are considered part of the experimental uncertainty budget $(\S 3.4)$, and they are not included in the Monte Carlo budget to avoid double counting.

\subsection{Sensitivity of transmission data (relative comparisons)}

Fig. 4.4 shows comparisons between measurements and EGSnrc calculations of the sensitivity of transmission data to a number of small changes in the experimental setup and in the operating parameters. The uncertainty on a given ratio is smaller than that on its components because many correlated Type-B uncertainties (e.g., those due to cross sections) cancel out. Overall, excellent agreement between measurements and calculations is obtained. The data suggest that, on average, EGSnrc is accurate for relative ion chamber response calculations at the $0.2 \%$ level. This supplements previous experimental validations at low energies ${ }^{82,83}$ and at high energies. ${ }^{84}$

\subsection{SENSITIVITY OF TRANSMISSION DATA (RELATIVE COMPARISONS)}




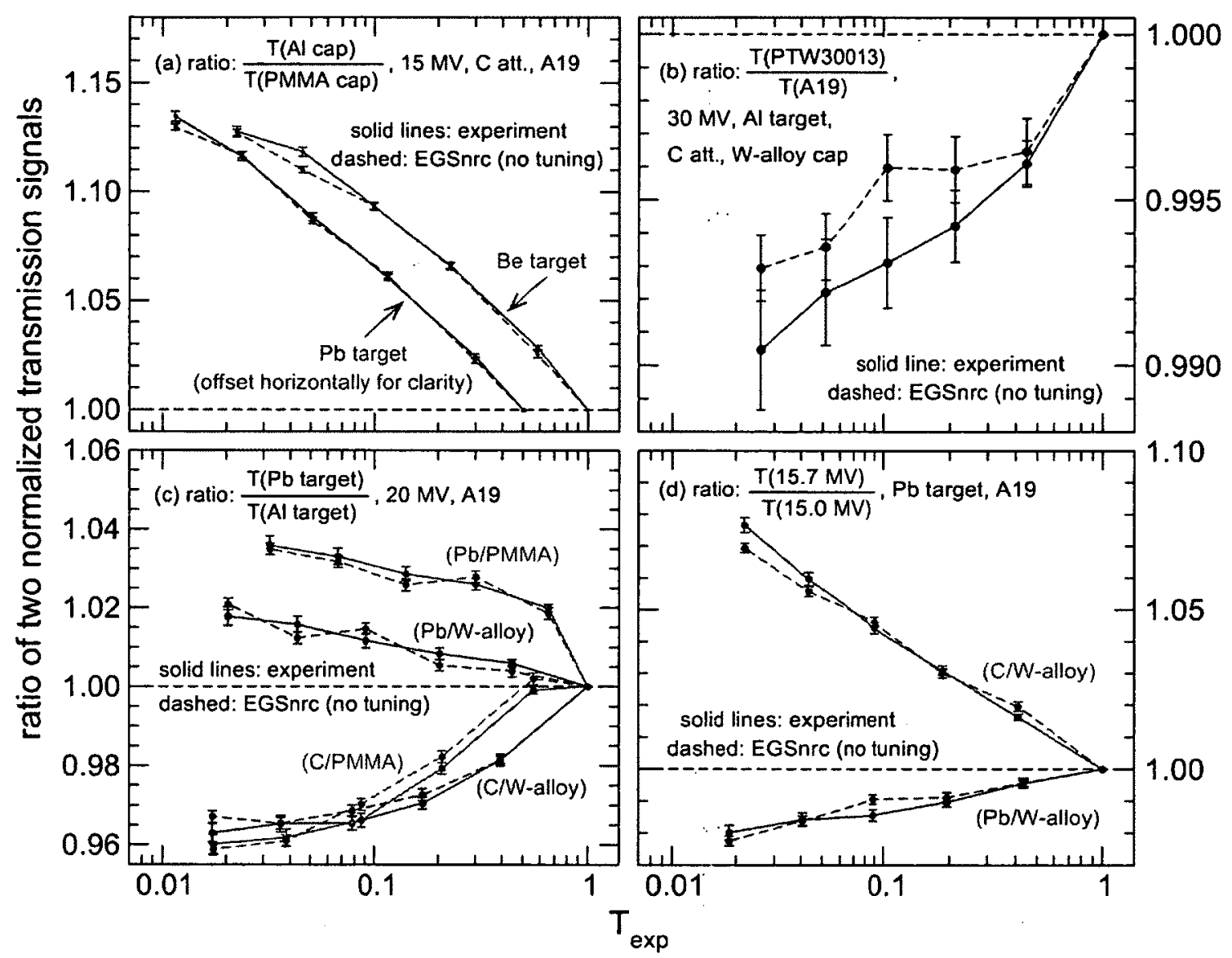

Figure 4.4: For the research linac beams, measurements and EGSnrc calculations of the sensitivity of transmission data to small changes in: (a) the material of the buildup cap, (b) the Farmer chamber construction details, (c) the bremsstrahlung target, and, (d) the incident electron energy. The abscissae are the experimental transmission data, $T_{\text {exp }}$, from the denominator of the ratio plotted in each panel. In panels (c) and (d), the pairs shown are different (attenuator/cap) combinations. In a given ratio, the correlation between the two transmission values makes the effect of many sources of uncertainties (including photon cross sections) cancel out. Therefore, for $T_{\text {exp }}$, the uncertainty bars are from the contribution of only the first three items in the budget in Table 3.2 (p. 55). For the EGSnrc data, only statistical uncertainties are relevant. Note the very different scales of the ordinates. From Paper III. 
In Fig. 4.4a, the large variation $(\sim 13 \%)$ in transmission for a relatively small change in the atomic number of the buildup cap material underlines the importance of accurate detector response modelling in transmission analysis. The agreement in Fig. 4.4a for the relative effect of the buildup cap is an indirect validation of the EGSnrc-calculated detector energy response for these caps (Fig. 2.2, p. 22), which is later used during spectral unfolding. The level of agreement in Fig. 4.4a is used to deduce an uncertainty component in Table 4.1 (p. 68) for the detector energy response.

Fig. 4.4b shows that even for similar Farmer-class $0.6 \mathrm{cc}$ chambers and the same buildup cap, transmission data are sensitive to the detector construction details, which indicates the importance of modelling such details. Given that the range of variation of transmission in Fig. 4.4b is only 1\%, the ability of EGSnrc calculations to accurately model that change is remarkable.

Fig. $4.4 \mathrm{c}$ demonstrates the sensitivity to the target material and the ability of EGSnrc to model it. The behavior is case-specific because of the interplay between the spectral shapes from the different targets, the detector energy response with different caps, and the energy dependence of the attenuation coefficient for different attenuators. For instance, the same four curves but for the $15 \mathrm{MV}$ beam are all above unity.

Fig. $4.4 \mathrm{~d}$ shows that a $4.7 \%$ change in the incident electron energy leads to a $\sim 7 \%$ change in transmission for $\mathrm{C}$ attenuators. The smaller effect with $\mathrm{Pb}$ attenuators $(\sim 2 \%)$ should not be misinterpreted as lack of energy sensitivity. Rather, it is because the $\mathrm{Pb}$ attenuation coefficient has a minimum at $\sim 2.5 \mathrm{MeV}$. Therefore even though the transmission values do not change significantly with energy, the contribution to them from photons at different energies does. The overall sensitivity to small energy changes is useful for accurate spectral unfolding. 


\subsection{Direct comparisons of transmission data}

An example of the measured and the EGSnrc-calculated transmission curves is shown in Fig. 4.5. The ratios of the EGSnrc-calculated transmission to the measured transmission are shown in Fig. 4.6 for all beams. Unlike the relative comparisons of Fig. 4.4, the comparisons here are affected by all the experimental and the Monte Carlo uncertainty components, in addition to cross section uncertainties. The overall agreement is excellent for a reduction in transmission by a factor of $\sim 60$, particularly that there is no tuning of the incident electron parameters in the EGSnrc model. For C attenuators, the agreement is better than $2 \%$ for all beams except for the $10 \mathrm{MV}$ beam (better than $3.4 \%$ ). For $\mathrm{Pb}$ attenuators, the agreement is typically better than $1 \%$.

As shown below in $\S 6.2$, uniformly scaling the photon cross sections used in the EGSnrc calculations (with the photonuclear effect included) by $+0.41 \%$ of unity for $\mathrm{C}$ and by $-0.02 \%$ of unity for $\mathrm{Pb}$ makes the majority of the data in Fig. 4.6 agree with unity within the uncertainty bars. These levels of uniform scaling of cross sections are well within the current estimates of photon cross section uncertainties. ${ }^{85}$ Therefore it is plausible to attribute the discrepancies beyond the uncertainty bars in Fig. 4.6 to cross section uncertainties. This excess discrepancy is used in $\S 6.2$ to deduce an estimate of photon cross section uncertainties that is more realistic than the currently used estimates for the energy range of this study.

Although cross section uncertainties alone are enough to explain the small discrepancies in Fig. 4.6, other possible explanations are explored here, but they are extremely difficult to verify because cross section errors are not known accurately. Fig. 4.6 shows that the agreement worsens for smaller transmission values, which is obvious for $\mathrm{C}$ and more subtle for $\mathrm{Pb}$. This trend is characteristic of a cross section effect, but can also be due to other effects that make a larger fraction of smaller transmission values (simi-

\subsection{DIRECT COMPARISONS OF TRANSMISSION DATA}




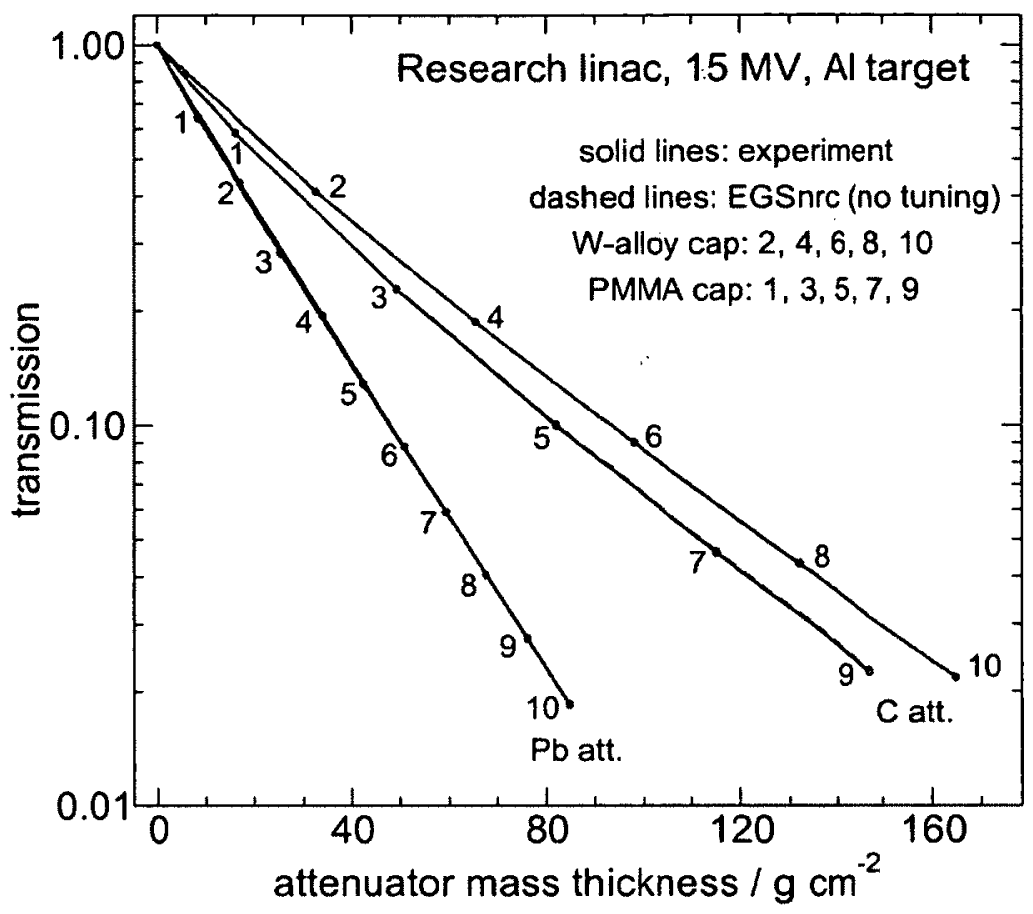

Figure 4.5: An example of the measured and the EGSnrc-calculated transmission curves for a given beam and different attenuator/buildup-cap combinations. The uncertainty bars are from Tables 3.2 (p.55) and 4.1 (p.68), and they are smaller than the size of the symbols. Photon cross section uncertainties are not included for the calculated transmission.

lar to the effects of $P_{p o l}$ or the attenuator mass thickness that are already accounted for). For $\mathrm{C}$ attenuators, EGSnrc results are always larger than experiment, which is reassuring that it is unlikely to be due to additional scatter effects that are unaccounted for (because they would have made the experimental results larger than the EGSnrc results). The agreement for the $10 \mathrm{MV}$ beam (the lowest stable MV beam on the linac) is clearly worse than that for other beams. This is reflective of the beam instability (and possibly other) issues for that beam. There are subtle hints of MV-clustering of the data in Fig. 4.6, which may indicate second-order beam-specific issues (related to the linac performance) that have not been accounted for experimentally. It might also indicate small energy dependence of cross section errors. 


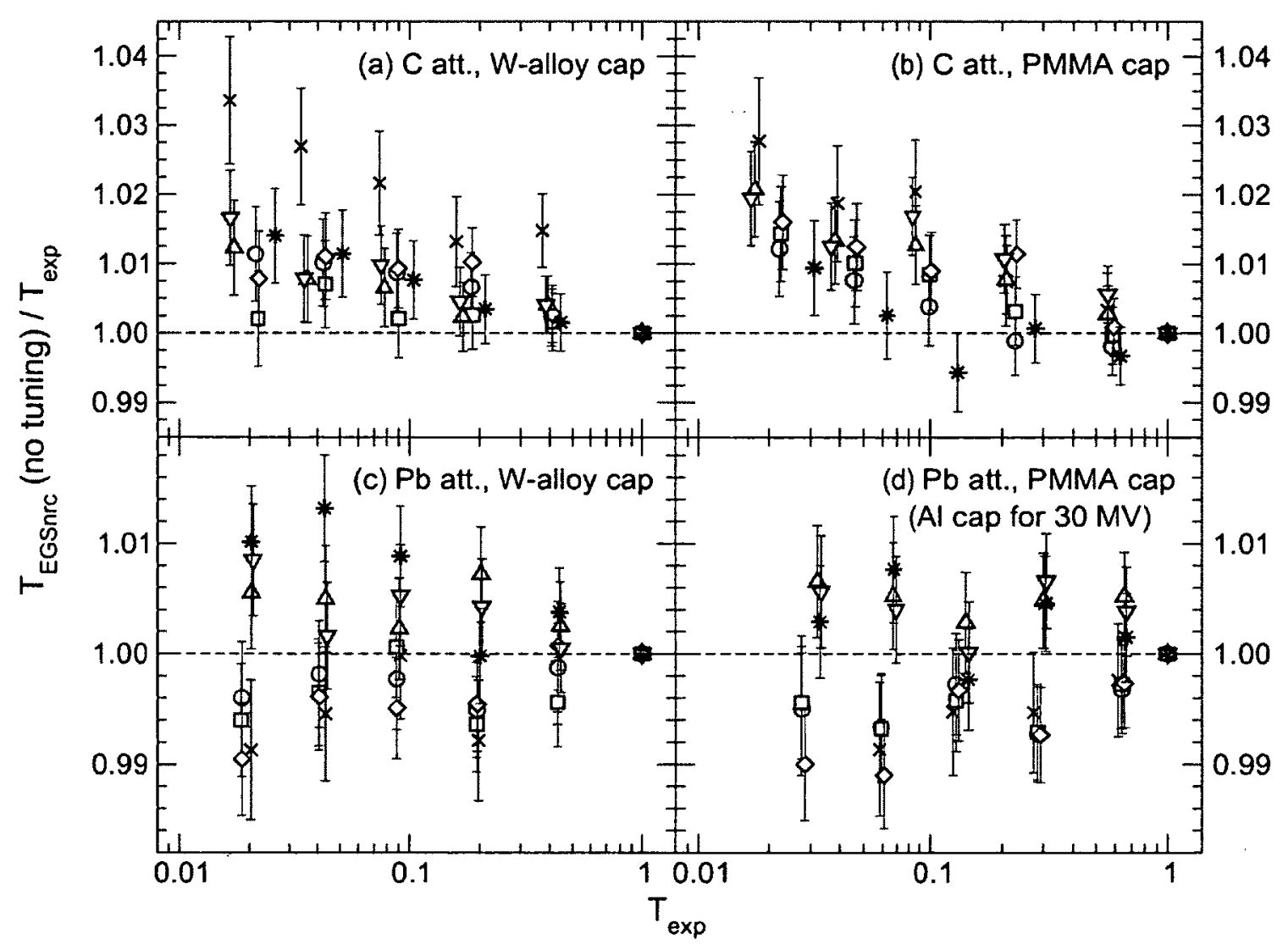

Figure 4.6: For the research linac beams, ratio of the transmission data calculated from a full EGSnrc model of the experiment, $T_{E G S n r c}$, to those measured experimentally, $T_{\text {exp }}$. Each panel includes data for the following $\mathrm{MV} /$ bremsstrahlung-target combinations: $10 \mathrm{MV} / \mathrm{Al}(\times), 15 \mathrm{MV} / \mathrm{Be}(\mathrm{O}), 15 \mathrm{MV} / \mathrm{Al}(\square), 15 \mathrm{MV} / \mathrm{Pb}(\diamond)$, $20 \mathrm{MV} / \mathrm{Al}(\triangle), 20 \mathrm{MV} / \mathrm{Pb}(\nabla)$, and $30 \mathrm{MV} / \mathrm{Al}(*)$. The $1 \sigma$ uncertainty bars are obtained by adding in quadrature the totals of the uncertainty budgets for $T_{\exp }$ (Table 3.2, p. 55) and $T_{E G S n r c}$ (Table 4.1, p. 68). Photon cross section uncertainties are not included for the calculated transmission. From Paper III. 


\subsection{Summary}

In this chapter, the measured transmission data for the research linac are used as a sensitive primary benchmark of EGSnrc. It is found that accurate transmission calculations require the use of the most accurate data and physics options available in EGSnrc, particularly the more accurate bremsstrahlung angular sampling option (a 5\% effect) and the modelling of photonuclear attenuation, which was added during this study (a 5.6\% effect). Relative transmission comparisons imply that EGSnrc is accurate within $0.2 \%$ for relative ion chamber response calculations. The relative comparisons indirectly validate the EGSnrc-calculated detector energy responses that are used in the next chapter during the unfolding. Direct comparisons of measured and EGSnrccalculated transmission data show agreement better than $2 \%$ for $\mathrm{C}(3.4 \%$ for the $10 \mathrm{MV}$ beam) and typically better than $1 \%$ for $\mathrm{Pb}$. The differences can be explained by acceptable photon cross section changes of $\leq 0.4 \%$ of unity. Given the small experimental and computational uncertainties, it can be concluded that cross section uncertainties are the ultimate limiting factor for any calculated transmission data. The full data needed for others to benchmark Monte Carlo codes are available on the web in Report III. 


\section{Chapter 5}

\section{Unfolded spectra}

\section{and incident electron energies}

In this chapter, the measured transmission data from $\mathrm{Ch} .3$ are used to unfold the spectra and the incident electron energies for the research and clinical beams. The unfolded data for the research beams constitute a direct independent validation of the proposed approach, while the unfolded data for the clinical beams demonstrate its application on a typical clinical linac. In $\S 5.1$ and $\S 5.2$, methods are presented to facilitate the validation of the unfolded spectra. The unfolding details are summarized in $§ 5.3$, including a clear definition of the role that Monte Carlo plays in the unfolding (to avoid potential confusion with the other Monte Carlo investigations done in this study). Then the results of the unfolded data and their validation are given for the research beams $(\S 5.4)$ and for the clinical beams $(\S 5.5)$.

\subsection{Research linac direct independent validation methods}

For the research linac beams, the differences between the transmission measurement setup and the previous NaI measurement setup ${ }^{6,11,12}$ are listed in Table 5.1. These differences need to be factored out to be able to validate the unfolded spectra against the NaI-measured spectra and their corresponding EGSnrc spectra. 
The first difference in Table 5.1 is the dose rate, which can affect the incident electron beam, and consequently the resulting bremsstrahlung spectra. However, for the research linac, the electron beam characteristics at the exit window are controlled by the settings of the combined magnet/slit system at the end of a very long drift tube. Therefore changes in the dose rate are unlikely to change the spectrum, and it is thus justified to benchmark the spectra unfolded from transmission data measured at near-therapeutic dose rates against the $\mathrm{NaI}$ spectra measured at ultralow dose rates.

The remaining differences in Table 5.1 lead to small differences in the spectra. EGSnrc simulation of the two setups show that the dominant contributor to spectral differences between the two setups is the difference in the pre-target materials. The RMS energy fluence deviation between the energy fluence spectra of the two setups is of the order of a few per cent. The beam with the largest RMS deviation (5\%) is that with the Be target. This is because, compared with other targets, the disparity is largest between the atomic number of $\mathrm{Be}$ and the effective atomic number of the pre-target materials. The spectral differences between the two setups are accounted for using a 'transfer' factor, $F_{T_{-} t o_{-} N_{a l}}$. For a given attenuator material and mass thickness, $F_{T_{-} t o_{-} N a I}$ is defined as the transmission value using the NaI setup parameters in Table 5.1, divided by the corresponding transmission value using the transmission setup parameters. The values of $F_{T_{-} t a_{-} N a I}$ are determined from Eq. 1.1 (p. 8) using $\psi(E)$ for the spectra seen by the detectors in both setups (pre-calculated using EGSnrc). The factor $F_{T_{-} t o_{-} \mathrm{NaI}}$ is called a 'transfer', rather than a 'correction', factor because it is specific to this primary benchmark which compares two experiments, and it is not needed for clinical measurements. The measured transmission data are multiplied by $F_{T_{-} t o-N a I}$ before the unfolding to enable the comparison between the unfolded spectra and their respective NaI-measured ones. Fig. 5.1 shows the variation of $F_{T_{-} t o_{-} N a l}$ with transmission. The transfer factor is generally small ( $\leq 2.5 \%$ from unity), except for the extreme case of a Be target discussed above (up to $5.1 \%$ from unity).

\subsection{RESEARCH LINAC DIRECT INDEPENDENT VALIDATION METHODS}


Table 5.1: For the research linac, differences between the transmission measurement setup and the previous $\mathrm{NaI}$ measurement setup. ${ }^{6,11,12}$. From Paper IV.

\begin{tabular}{|c|c|c|}
\hline Aspect of difference & NaI setup & Transmission setup \\
\hline Dose Rate at $\sim 3 \mathrm{~m}$ & $\sim 1$ photon/pulse & $\sim 40 \mathrm{cGy} / \mathrm{min}$ \\
\hline Pre-target material & $\begin{array}{l}0.013 \mathrm{~cm} \text { pure Ti exit window } \\
+0.01(5)^{a} \mathrm{~cm} \text { pure Si monitor } \\
+0.0051 \mathrm{~cm} \text { steel chamber wall } \\
+1.685 \mathrm{~cm} \text { air }\end{array}$ & $\begin{array}{l}0.00412 \mathrm{~cm} \mathrm{Ti} \text { alloy exit } \\
\text { window, } 4.42 \mathrm{~g} / \mathrm{cm}^{3} \\
(90 \% \mathrm{Ti}, 6 \% \mathrm{Al}, 4 \% \mathrm{~V}) \\
+2.05 \mathrm{~cm} \text { air }\end{array}$ \\
\hline Three target thicknesses & & \\
\hline (a) $\mathrm{Pb}$ for $10,20 \mathrm{MV}$ & $0.805,1.008 \mathrm{~cm}$ & $0.793,1.016 \mathrm{~cm}$ \\
\hline (b) Al for $30 \mathrm{MV}$ & $6.00 \mathrm{~cm}$ & $6.60 \mathrm{~cm}$ \\
\hline Two incident $\mathrm{MeV}$ & $15.18,30.45$ & $15.00,30.00$ \\
\hline Energy spread & Gaussian, $\sigma=0.64 \%$ & Gaussian, $\sigma=0.4 \%$ \\
\hline Radial spread & uniform circle, dia. $0.35 \mathrm{~cm}$ & Gaussian, $1 \mathrm{~mm}$ FWHM \\
\hline Divergence & assumed none in Ref. 6 & $0.03^{\circ}$, apex $1 \mathrm{~m}$ upstream \\
\hline Cone half angle & $0.22^{\circ}$ & $0.29^{\circ}$ \\
\hline
\end{tabular}

${ }^{a} \mathrm{It}$ is $0.010 \mathrm{~cm}$ for the Be target and $0.015 \mathrm{~cm}$ for the $\mathrm{Al}$ and $\mathrm{Pb}$ targets

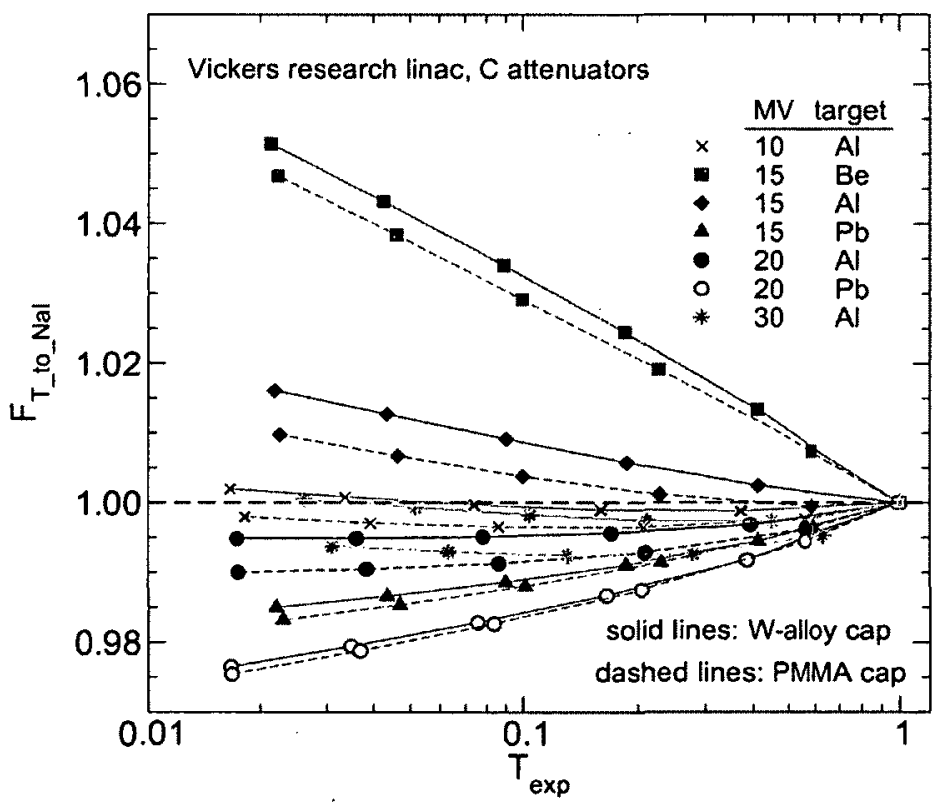

Figure 5.1: For the research linac beams, the 'transfer' factor $F_{T_{-} t o_{-} \mathrm{NaI}}$ versus the experimental transmission, $T_{\text {exp }}$, for $\mathrm{C}$ attenuators. Except for the Be target, the transfer factor differs from unity by $\leq 2.5 \%$. The corresponding transfer factors for $\mathrm{Pb}$ attenuators are smaller ( $\leq 1.6 \%$ from unity). From Paper IV. 


\subsection{Clinical linac validation methods}

Unlike the research beams, direct independent validation is not available for the clinical beams. Therefore the unfolded quantities are validated using the two methods described below. The two methods combined constitute a rigorous test of the unfolding accuracy. First validation method: This method does not involve using the unfolded spectral shape. It is meant to validate the unfolded electron energy only. In this method, BEAMnrc $^{37,38} /$ cavity $^{39}$ are used to calculate transmission values from a full model of the experimental setup, and these data are compared to the measured transmission signals. The incident electron energies used in the EGSnrc simulations are the unfolded values from the transmission measurements $(\S 5.5)$. The other needed electron parameters are taken from Tonkopi et $a l^{3}$ who estimated these parameters using in-air off-axis ratio measurements on the same linac. Those other parameters are: (a) energy spread: none, (b) focal spot: Gaussian with FWHM values of $0.15,0.05$ and $0.19 \mathrm{~cm}$ for the 6, 10, and $25 \mathrm{MV}$ beams, respectively, and, (c) mean angular divergence: none for the 6 and $10 \mathrm{MV}$ beams and $1.15^{\circ}$ for the $25 \mathrm{MV}$ beam. Photonuclear attenuation is included everywhere except in the detector. Its effect at $25 \mathrm{MV}$ on the smallest calculated transmission values is $1.5 \%$ for $\mathrm{C}$ attenuators and $2 \%$ for $\mathrm{Pb}$; the effect is negligible for the 6 and $10 \mathrm{MV}$ beams.

Second validation method: In this method, BEAMnrc is used to model the setup to extract the primary photon spectra at the attenuator surface. The unfolded spectra are compared to the BEAMnrc spectra. As in the first method, EGSnrc simulations use the unfolded electron energies in combination with the other electron parameters from above. By definition, the EGSnrc spectra and the unfolded spectra have an identical endpoint energy. Therefore this method is meant to validate the unfolded spectral shape, as a complement to the first method. 


\subsection{Unfolding using experimental data}

The unfolding details are the same as those given earlier in $\S 2.2$ (hence not reproduced here), except that the input transmission data are experimental, rather than computational. The following details are in addition to those of $\S 2.2$.

For a given MV, the data of the four measured transmission curves from the different attenuator/cap combinations are fed simultaneously to the unfolding algorithm. For the research linac beams, the experimental data are multiplied by the transfer factor $F_{T_{-} t o_{-} \mathrm{NaI}_{\mathrm{I}}}$ before being used as input. For the beams of both linacs, the variances for weighting the input data are based on the final results of the experimental uncertainty budgets (including the uncertainties in the electron source parameters for the research linac beams). Photonuclear attenuation is included in the total photon cross sections ( $(2.6)$. The energy fluence spectrum is specified using the new functional form (Eq. 2.1, p. 17). The incident electron energy is one of the four free parameters of the function, and it is searched for concurrently with the other three (except for the $30 \mathrm{MV}$ beam of the research linac, where an exhaustive grid $\operatorname{search}^{86}$ on the electron energy was necessary). The minimization is robust against different initial estimates of the free parameters, including the electron energy (within many $\mathrm{MeV}$ of the nominal MV). No a-priori knowledge of the linac head details is required.

Non-ideal attenuation is corrected for using the iterative method of $\S 2.7$. The method does not require knowledge of the linac head details. It uses the unfolded spectrum as a point source in EGSnrc calculations to estimate a correction factor, $F_{\text {non_ideal }}$, that accounts for the combined effect of non-ideal conditions. The experimental transmission data are computationally corrected by $F_{\text {non-ideal }}$, and a revised spectrum is unfolded. In practice, only one iteration is needed for the spectrum to converge. Fig. 5.2 shows examples of the estimated $F_{\text {non-ideal }}$ after one iteration (up to a $1.5 \%$ correction).

\subsection{UNFOLDING USING EXPERIMENTAL DATA}




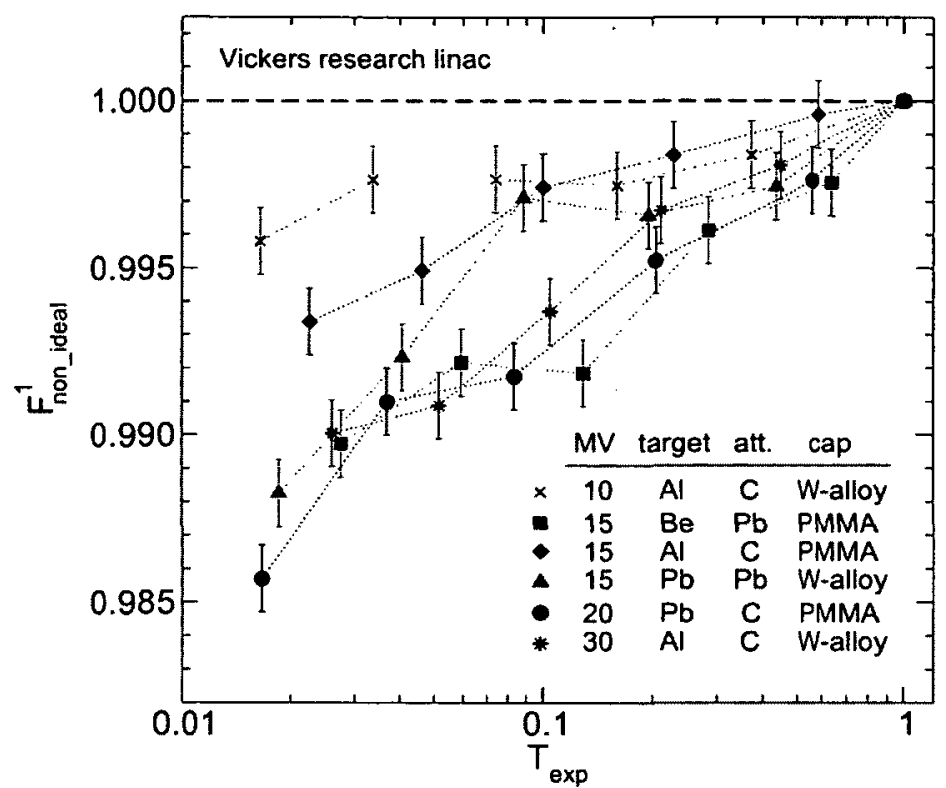

Figure 5.2: For the research linac beams, the correction factor $F_{\text {non_ideal }}^{1}$ versus the experimental transmission, $T_{\text {exp }}$, after one iteration (hence the superscript 1). The non-ideal corrections for the clinical beams are comparable. From Paper IV.

With 16 degrees of freedom (20 measurement points minus 4 free parameters), the typical final reduced $\chi_{\min }^{2}$ between the input experimental transmission signals and those calculated using the final free parameters is lower than unity. Given that the spectral functional form is not over-parameterized, a lower-than-unity $\chi_{\min }^{2}$ implies that the experimental uncertainty budget is conservative - possibly from ignoring the correlation between the uncertainty components, and/or from using extreme effects to deduce some components.

Monte Carlo plays an auxiliary but important role in the spectral unfolding in only two areas: detector energy response modelling, and corrections for non-ideal attenuation. The Monte Carlo-calculated detector response is experimentally validated ( $\S 4.5)$, and the correction for non-ideal conditions is not very sensitive to the spectrum used to estimate it $(\S 2.7)$. Therefore the unfolded spectra do not have a significant Monte Carlo bias, and can thus be considered independent experimental spectra.

\subsection{UNFOLDING USING EXPERIMENTAL DATA}




\subsection{Unfolded data for the research linac}

For the research beams, comparisons of the unfolded spectra and the unfolded incident electron energies, $E_{e}$, against the benchmark data are shown in Fig. 5.3 and Table 5.2. Normalization: The spectra in figure 5.3, and the RMS energy fluence deviations in Table 5.2 are based on normalization of each spectrum to unit energy fluence. This method of normalization gives more reasonable weight to the higher energy portion of the spectrum compared with normalization to unit fluence, and graphically reveals the discrepancies better. It also avoids the potential bias from normalization to a specific point (e.g., the peak).

Confidence bounds: In figure 5.3, the $95 \%$ confidence bounds are determined by the variances used for weighting the input transmission signals during the minimization. The bounds include the effect of the uncertainties in the electron source parameters, and the effect of other experimental systematic uncertainties. However, they do not include the effect of cross section uncertainties or the systematic errors from the functional form. The bounds are generally tight, which is a direct result of the small experimental uncertainties. For the unfolded $30 \mathrm{MV}$ spectrum, the bounds are larger because the incident electron energy is obtained through a grid search, where the correlation between the electron energy and the other free parameters is missing, which is found empirically to lead to more conservative uncertainty bounds. The bounds have an irregular shape, typically with two necks. This is a result of conforming the spectral shape to the functional form, where some energy fluence values are more likely to be crossed by the unfolded spectrum than others.

Accuracy of the unfolded spectra: The $95 \%$ confidence bounds on the unfolded spectra mostly fall within the statistical noise of the $\mathrm{NaI}$ data. The comparison of the unfolded spectra against the high precision EGSnrc spectra also show good agreement, with some 


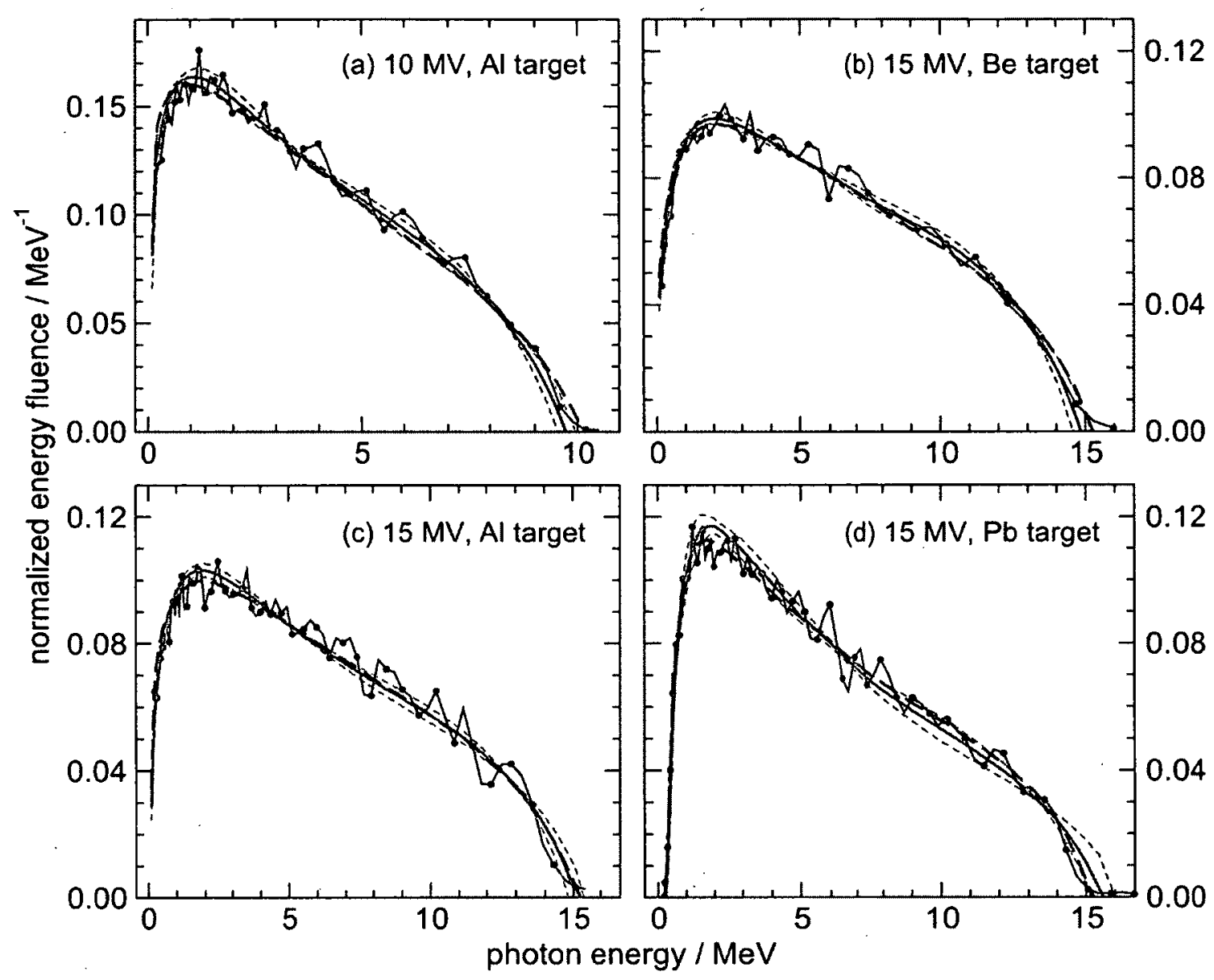

Figure 5.3: For the research linac beams, comparison of the spectra unfolded from transmission measurements against the benchmark NaI-measured spectra ${ }^{6,11,12}$ and their corresponding EGSnrc spectra. The NaI-measured spectra are the thin solid lines with every other data point shown as a small circle. The EGSnrc spectra are the thick long-dashed lines, with negligible statistical uncertainty. The unfolded spectra are the thick solid lines, with the $95 \%$ confidence bounds shown as two thin short-dashed lines. The EGSnrc spectra and the unfolded spectra are often visually indistinguishable from each other. Spectra are normalized to unit energy fluence. Panels (f), (g), and (h) are shown on the following page. From Paper IV. 


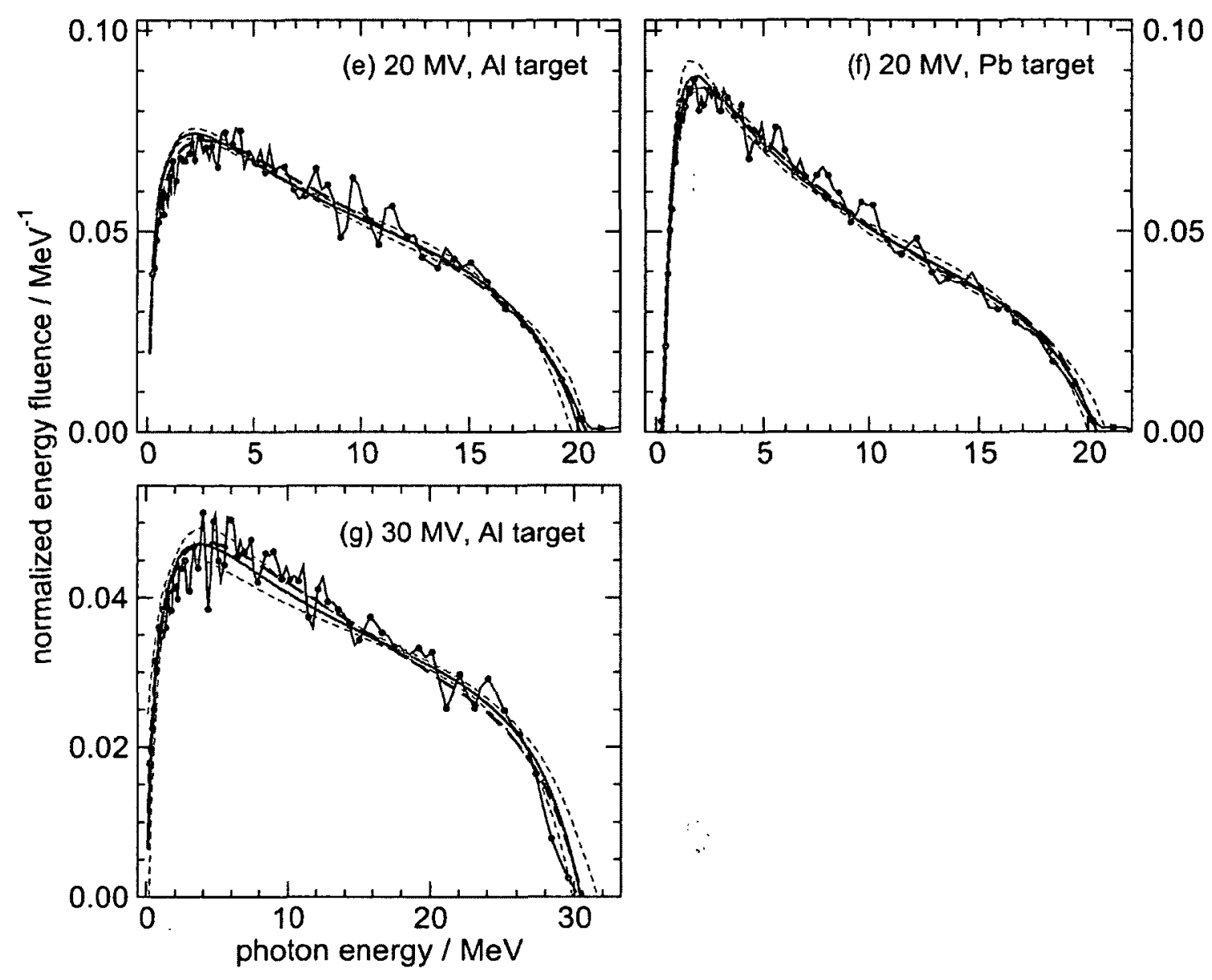

Figure 5.3: ... continued from the previous page. 
Table 5.2: For the research linac beams, evaluation of the accuracy of the unfolded energy-fluence spectra, $\psi$, and the unfolded incident electron energies, $E_{e}$. For the differences between the unfolded and the EGSnrc spectra, $\Delta \psi$ is the RMS energy fluence deviation (normalized to the mean energy fluence), and $\Delta E_{\text {mean }}$ is the difference in the mean energy. For the incident electron energy, $\Delta E_{e}$ is the relative difference in $E_{e}$ between the unfolded and the bending magnet values. The bending magnet values are those used during the NaI measurements. ${ }^{6,11,12}$ From Paper IV.

\begin{tabular}{|c|c|c|c|c|c|c|}
\hline MV & $\begin{array}{l}\text { Brem. } \\
\text { target }\end{array}$ & $\begin{array}{l}\Delta \psi \\
(\%)\end{array}$ & $\begin{array}{c}\Delta E_{\text {mean }} \\
(\mathrm{MeV})\end{array}$ & $\begin{array}{c}E_{e}(\text { magnet }) \pm 1 \text { s.d. } \\
\quad(\text { both in } \mathrm{MeV})\end{array}$ & $\begin{array}{c}E_{e} \text { (unfolded) } \pm 1 \text { s.d. } \\
\quad(\text { both in } \mathrm{MeV})\end{array}$ & $\begin{array}{l}\Delta E_{e} \\
(\%)\end{array}$ \\
\hline 10 & $\mathrm{Al}$ & 4.5 & 0.02 & $10.09 \pm 0.04$ & $9.73 \pm 0.11$ & -3.5 \\
\hline \multirow[t]{3}{*}{15} & $\mathrm{Be}$ & 4.8 & 0.09 & $15.18 \pm 0.06$ & $14.84 \pm 0.19$ & -2.3 \\
\hline & $\mathrm{Al}$ & 4.8 & 0.10 & & $15.13 \pm 0.13$ & -0.3 \\
\hline & $\mathrm{Pb}$ & 5.5 & 0.02 & & $15.59 \pm 0.19$ & 2.7 \\
\hline \multirow[t]{2}{*}{20} & $\mathrm{Al}$ & 4.0 & -0.11 & $20.28 \pm 0.08$ & $20.10 \pm 0.21$ & -0.9 \\
\hline & $\mathrm{Pb}$ & 5.1 & -0.11 & & $20.38 \pm 0.22$ & 0.5 \\
\hline 30 & $\mathrm{Al}$ & 3.1 & 0.12 & $30.45 \pm 0.12$ & $30.44 \pm 0.63$ & -0.0 \\
\hline
\end{tabular}

small differences. The RMS energy fluence deviation is typically $4.5 \%$ (the average of the data in the third column of Table 5.2), and the deviations in the mean spectrum energy are less than $150 \mathrm{keV}$. There are several reasons that contribute to the differences (e.g., around the peaks and in other regions) between the EGSnrc and the unfolded spectra beyond the confidence bounds. (a) Different normalization methods lead to different positioning of the spectra relative to each other and hence the regions of discrepancy could change. (b) Systematic errors from conforming the spectral shape to a four-parameter functional form, which are not part of the confidence bounds shown N.B., from $\S 2.1$, when the function is fit directly to a variety of high-precision spectra, there is an average of $1.7 \%$ RMS energy fluence deviation. (c) Uncertainties in the photon cross sections that are used during the unfolding (i.e., $\frac{\mu}{\rho}(E)$ in Eq. 1.1, p. 8). (d) The accuracy of the EGSnrc benchmark spectra, which are affected by second-order limitations in the underlying physics models (e.g., the bremsstrahlung energy-angle relations) - an indication of this possibility is that the spectra generated by different mature Monte Carlo codes show some differences. ${ }^{6}$

\subsection{UNFOLDED DATA FOR THE RESEARCH LINAC}


Accuracy of the unfolded electron energies: From Table 5.2, the comparison of the unfolded $E_{e}$ values against their respective bending magnet values show that the accuracy of unfolding the incident electron energy is $\sim 3 \%$. For the measurements with electron beams of energies 15.00 and $15.70 \mathrm{MeV}$ (a $4.7 \%$ energy change) incident on a $\mathrm{Pb}$ target, the unfolded electron energies are, respectively, $15.4 \pm 0.2 \mathrm{MeV}(1 \mathrm{s.d}$.) and $16.3 \pm 0.2 \mathrm{MeV}$ ( 1 s.d.). The unfolded values are within $\sim 3 \%$ of their true values, and they are outside the $95 \%$ confidence bounds of each other. This confirms the $\sim 3 \%$ resolving power of the technique.

Detector independence: To test the independence of the unfolded spectra from the detector, the input transmission data for the PMMA cap are replaced with those for the Al cap (in combination with the W-alloy cap data in both cases), and the corresponding detector energy response is used during the unfolding. Strong overlap is observed between the confidence bounds of the spectra unfolded using the two data sets. The RMS energy fluence deviations between any two of such unfolded spectra are smaller than the overall accuracy of the technique (viz., the $4.5 \%$ average value from Table 5.2). The same results are obtained when the data for the Exradin A19 are replaced with those for the PTW30013 chamber (with its corresponding energy response used during the unfolding). These results indicate that the unfolded spectra do not have systematic detector-related bias.

Sensitivity investigation: Figure 5.4 illustrates the effect of different variations of the input data on the unfolding accuracy. From Table 5.2, the RMS energy fluence deviation, $\Delta \psi$, for the $20 \mathrm{MV}$ beam from a $\mathrm{Pb}$ target is $5.1 \%$. When only one attenuator that has a monotonic attenuation coefficient, (i.e., C), and one detector (A19 with Walloy cap) are used (which is the approach used in previous studies), $\Delta \psi$ worsens to $11.2 \%$, even with the high accuracy of the transmission measurements and the use of the four-parameter spectral functional form. When the polarity correction is ignored, 


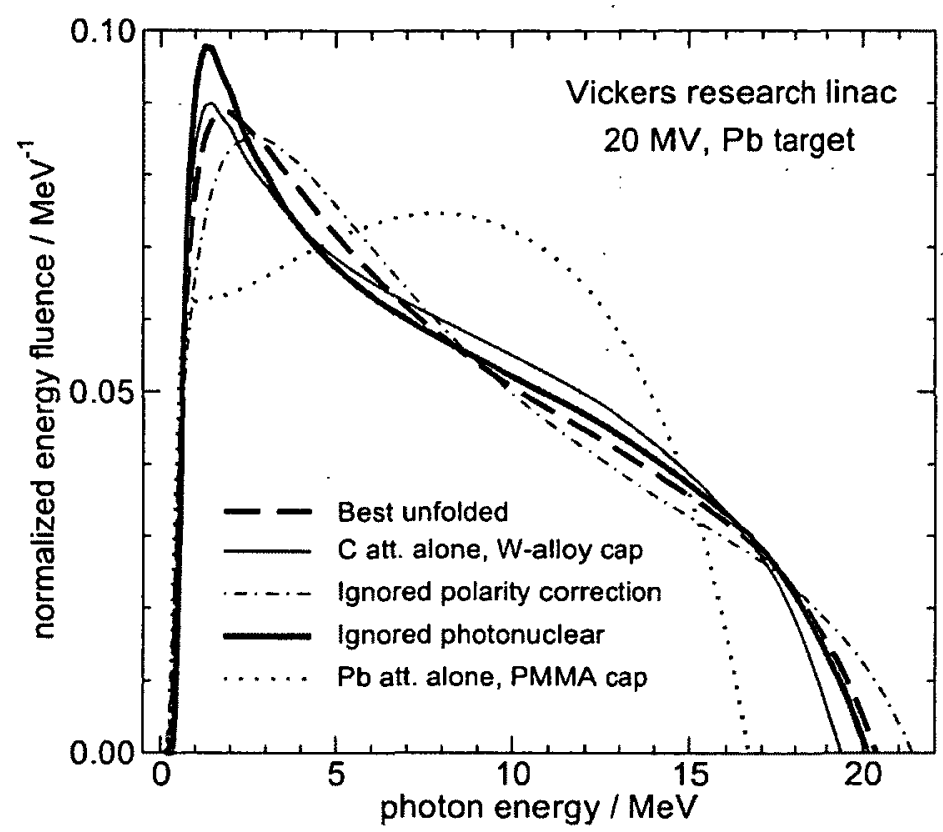

Figure 5.4: For the $20 \mathrm{MV}$ beam of the research linac, the figure shows the effects on the accuracy of the unfolded spectra when using different variations of the input transmission data and when ignoring different influence quantities. The 'best unfolded' spectrum is that from Fig. 5.3f, and it is very close to the EGSnrc benchmark spectrum (as seen in Fig. 5.3f). The other four curves are the unfolded spectra for the situations shown in the legend. Spectra are normalized to unit energy fluence. From Paper IV.

$\Delta \psi$ worsens from $5.1 \%$ to $8.9 \%$. When the photonuclear effect is ignored, $\Delta \psi$ worsens from $5.1 \%$ to $14.9 \%$. The examples in figure 5.4 underline the importance of the various computational and experimental effects investigated. Sensitivity studies for all beams indicate that the improvement over previous studies in the spectral unfolding accuracy is about a factor of 3 (on top of the improvements from the use of the new functional form and the accurate detector energy response). As a theoretical exercise, if $\mathrm{Pb}$ is the only attenuator used, the unfolding becomes degenerate by definition (because the attenuation coefficient for $\mathrm{Pb}$ is not monotonic in the energy range of interest), and wrong spectra are likely, as seen in Fig. 5.4. 


\subsection{Unfolded data for the clinical linac}

For the Elekta Precise beams, the unfolded quantities and their validation (using the two methods discussed in §5.2) are shown in Figs. 5.5 and 5.6, and in Table 5.3. The details of the normalization and the confidence bounds are the same as those for the research linac beams $(\S 5.4)$, except that the confidence bounds do not account for the uncertainties on the incident electron parameters.

First validation method: This method validates the unfolded electron energy only, $E_{e}$. Fig. 5.5a-c show that the EGSnrc transmission data, which are calculated using the unfolded incident electron energies, agree with the measured signals within $\sim 1.5 \%$ (three times the uncertainty on the ratios). This can be caused by several uncertainty components that are not part of the error bars in Fig. 5.5, including: (a) the uncertainty on the unfolded electron energy; (b) the uncertainties on the other electron source parameters from Tonkopi et al; (c) the uncertainties in the photon cross sections in EGSnrc calculations; and, (d) the accuracy of the relevant physics models in EGSnrc.

Tonkopi et $a l^{3}$ extracted the incident electron energies for the same linac using two methods: (a) in-air off-axis ratio measurements, and, (b) a combination of electron beam depth-dose data and current settings for the linac bending magnet. Their estimates are shown in Table 5.3. The unfolded $E_{e}$ values in the current study differ from the off-axis ratio estimates by $+6.3 \%,-1.2 \%$, and $+1.5 \%$ for the 6,10 , and $25 \mathrm{MV}$ beams, respectively. This is investigated here for the case with the largest difference (i.e., $6 \mathrm{MV}$ ). The estimate of $E_{e}$ from Tonkopi et al (i.e., $5.75 \mathrm{MeV}$ ) is used in EGSnrc simulations of the transmission setup to calculate the corresponding transmission values. Fig. 5.5d shows that the calculated transmission values differ from the measured signals by up to $9.7 \%, 5.0 \%$ and $2.7 \%$ for the $\mathrm{C}, \mathrm{Cu}$, and $\mathrm{Pb}$ attenuators, respectively. The corresponding differences when using the value of $E_{e}$ unfolded in the current study are within $1 \%$ for 


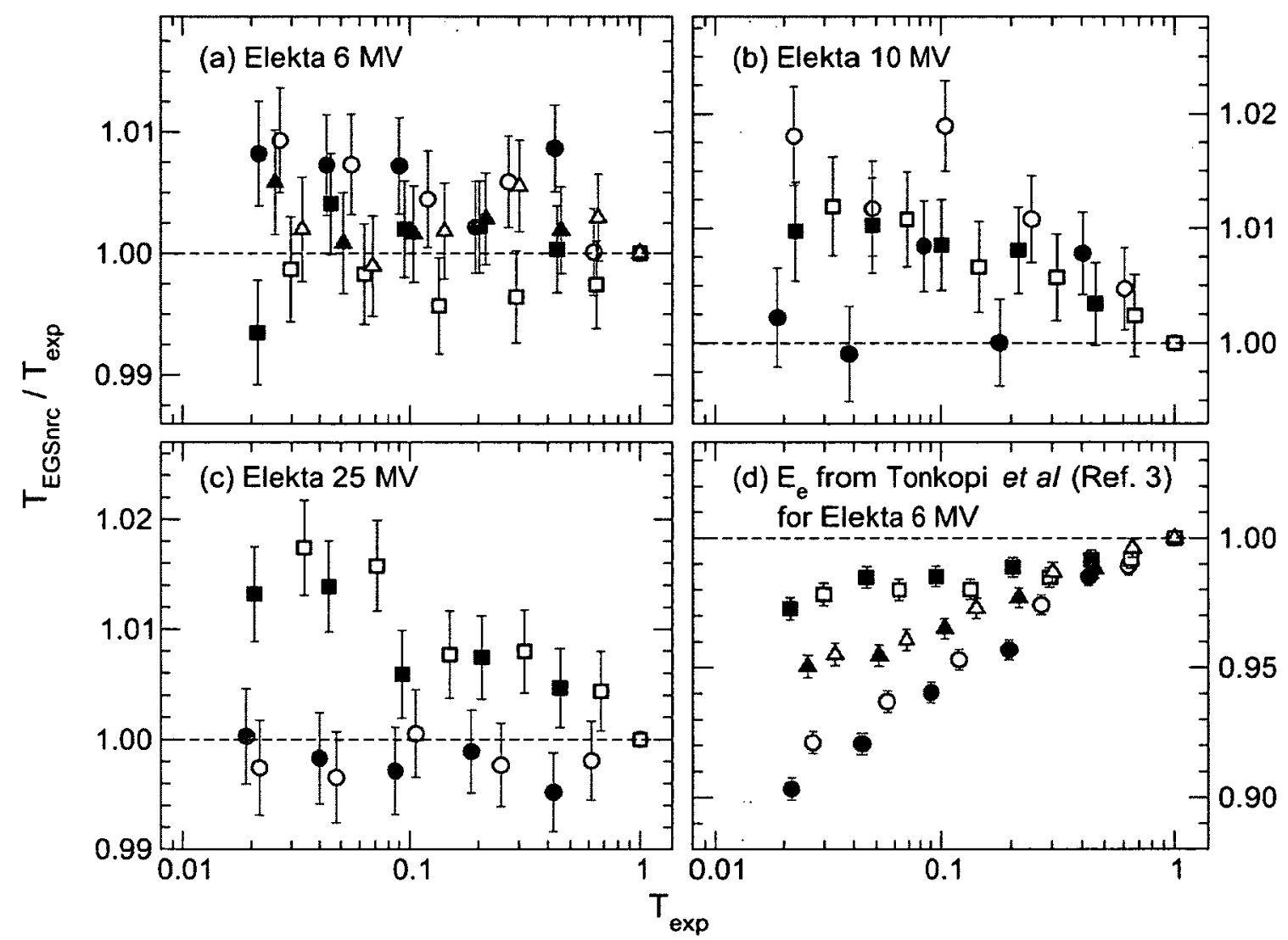

Figure 5.5: For the clinical linac (an Elekta Precise), panels (a) to (c) show the comparison of the EGSnrc transmission values, $T_{E G S n r c}$ (calculated using the unfolded incident electron energies, $E_{e}$ ), to the experimental transmission signals, $T_{\text {exp }}$. The data are for the following attenuator/buildup-cap combinations: C/W-alloy ( ) C/PMMA (O), $\mathrm{Pb} / \mathrm{W}$-alloy (ם), Pb/PMMA ( $\square$ ), $\mathrm{Cu} / \mathrm{W}$-alloy $(\boldsymbol{\Delta})$ and $\mathrm{Cu} / \mathrm{PMMA}(\Delta)$. Panel (d) shows the same comparison as in panel (a) but when using the $E_{e}$ value estimated by Tonkopi et $a^{3}$ from in-air off-axis ratio measurements on the same linac. The data equivalent to panel (d) for the 10 and $25 \mathrm{MV}$ beams show significantly smaller differences ( $\leq 3.3 \%$ from unity). Note the very different scale of the ordinate of panel (d). From Paper IV. 


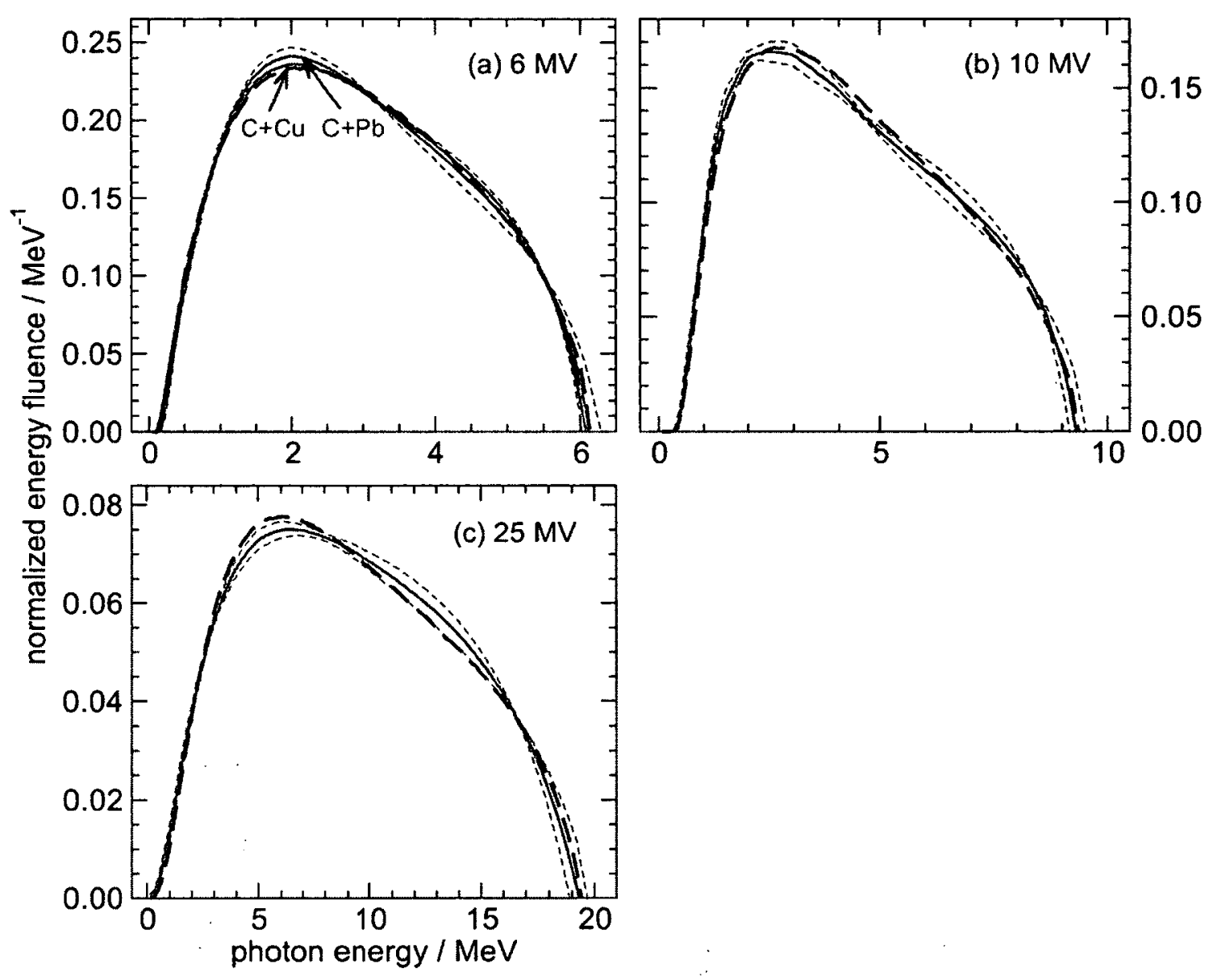

Figure 5.6: For the clinical linac (an Elekta Precise), comparison of the spectra unfolded from measured transmission signals (thick solid lines) against the EGSnrc spectra (thick long-dashed lines) calculated using the unfolded incident electron energies. The $95 \%$ confidence bounds on the unfolded spectra are the two thin short-dashed lines. All spectra are unfolded from the combined data of $\mathrm{C}+\mathrm{Pb}$ attenuators, except for an additional spectrum in panel (a) which is unfolded using $\mathrm{C}+\mathrm{Cu}$ data. Spectra are normalized to unit energy fluence. From Paper IV. 
Table 5.3: For the clinical linac beams, comparison of the unfolded spectra against the EGSnrc spectra. The definitions of $\Delta \psi$ and $\Delta E_{\text {mean }}$ are the same as in Table 5.2 (p. 84). Also shown are the estimated $E_{e}$ values from other studies on the same linac. From Paper IV.

\begin{tabular}{|c|c|c|ccc|}
\hline & & & \multicolumn{3}{|c|}{$E_{e}(\mathrm{MeV}) \pm 1$ s.d. $(\mathrm{MeV})$} \\
\cline { 4 - 6 } MV & $\Delta \psi$ & $\Delta E_{\text {mean }}$ & $\begin{array}{c}\text { Unfolded, } \\
\text { this study }\end{array}$ & $\begin{array}{c}\text { From off-axis } \\
\text { ratios, Ref. 3 }\end{array}$ & $\begin{array}{c}\text { From e } \\
\text { dose, Ref. }\end{array}$ \\
\hline 6 & 2.8 & 0.01 & $6.1 \pm 0.1$ & 5.75 & $5.7 \pm 0.4$ \\
10 & 4.0 & -0.06 & $9.3 \pm 0.1$ & 9.4 & $9.0 \pm 0.4$ \\
25 & 4.4 & -0.33 & $19.3 \pm 0.2$ & 19.0 & $19.9 \pm 0.7$ \\
\hline
\end{tabular}

all attenuators (Fig. 5.5a), which indicates that it is a more realistic estimate of $E_{e}$. This is also supported by an unpublished extension of the recent work on the effective point of measurement. ${ }^{87}$ In this extension, Tessier found that an $E_{e}$ value of $6.0 \mathrm{MeV}$ leads to better agreement than $5.75 \mathrm{MeV}$ between measured and EGSnrc-calculated depth-dose curves (including a faithful detector model) [Tessier, 2012 - personal communication]. The level of agreement in Fig. 5.5a-c loosely suggests that the other electron parameters, which are taken from Tonkopi et $a l^{3}$ are acceptable. The exercise just performed to test the effect on simulated transmission signals when using different $E_{e}$ values shows strong sensitivity, which is a useful attribute of the validation method.

Second validation method: This method validates the spectral shape only. Fig. 5.6 shows good agreement between the unfolded and the EGSnrc-calculated spectra, with a typical RMS energy fluence deviation of $3.7 \%$ (the average of the data in the second column of Table 5.3). The possible reasons for the discrepancies are the same as those for the research linac beams ( $\$ 5.4)$, in addition to the uncertainties in the incident electron parameters, which are unknown for the clinical linac beams. Fig. 5.6a for the $6 \mathrm{MV}$ beam shows that when the data for the $\mathrm{C}+\mathrm{Cu}$ attenuators are used, the unfolded spectrum is very close to that with the $\mathrm{C}+\mathrm{Pb}$ data. This indicates that the unfolded spectra do not have systematic attenuators-related bias, provided that the attenuation coefficient for at least one of the attenuators is monotonic in the energy range of interest. 


\subsection{Higher transmission cutoffs}

It was shown computationally in $\S 2.5$ that when transmission data down to only $10 \%$ (as opposed to $1-2 \%$ ) are used to unfold spectra, there is only modest reduction in accuracy ( $\Delta \psi$ worsens from $2.3 \%$ to $3.2 \%$ ), with some widening in the confidence bounds. This is tested experimentally using the research linac data, and similar results are obtained. Using a $10 \%$ cutoff significantly reduces the magnitude and the uncertainty of many computational and experimental influence quantities. However, it reduces energy differentiation and thus increases the demand on measurement accuracy. A cutoff much higher than $10 \%$ is found to reduce the robustness, and the results become case-specific. Accurate measurements down to $\sim 1-2 \%$ remain useful for more energy differentiation, tighter confidence bounds, more rigorous benchmarking for Monte Carlo codes (Ch.4), and evaluation of photon cross section uncertainties (Ch.6).

\subsection{Summary}

In this chapter, the approach proposed in Ch. 2 is validated experimentally using the measured transmission data for the research linac beams. Energy fluence spectra are unfolded with RMS energy fluence deviations of $4.5 \%$, and the accuracy of unfolding the electron energy is $\sim 3 \%$. This level of accuracy is at least a factor of 3 over the best previous studies. The unfolding does not require knowledge of the linac head or the electron energy. For the unfolding, Monte Carlo plays an important auxiliary role in precalculating the detector energy response, and in correcting for some nonideal attenuation conditions. Accurate unfolding can be achieved with a transmission cutoff of only $10 \%$, provided that the other components of the proposed approach are implemented. The practicality of the proposed approach is successfully demonstrated on a typical clinical

linac. Photon cross section uncertainties are the ultimate limiting factor of the unfolding accuracy, and they are estimated in the following chapter.

\subsection{HIGHER TRANSMISSION CUTOFFS}




\section{Chapter 6}

\section{Estimating photon}

\section{cross section uncertainties}

Recent studies have shown that the uncertainties in photon cross sections at radiation therapy energies play an important role in determining the overall uncertainty when calculating fundamental dosimetric quantities such as beam quality conversion factors ${ }^{73,88}$ and air kerma standards. ${ }^{89}$ In transmission analysis, these uncertainties are particularly important because they are weighted by the attenuator mass thickness in the exponent, which leads to large effects on smaller transmission values. To illustrate this, for a monoenergetic photon beam, a fractional cross section error, $f$, leads to an error of $T^{f}$ on the calculated transmission, $T$. Thus for a $1.5 \%$ cross section error and a two-order-ofmagnitude attenuation, the error on $T$ would be $0.01^{0.015}=7.2 \%$. The uncertainties in the photon cross sections used during spectral unfolding ( $\frac{\mu}{\rho}$ in Eq. 1.1, p. 8) affect the accuracy of the unfolded spectra, particularly that the problem is ill-posed. In radiation protection applications, the signal is attenuated by three or more orders of magnitude, and cross section uncertainties are amplified even more. ${ }^{90}$

The current estimate of photon cross section incertainties is given by Hubbell, ${ }^{85}$ who recognized the difficulty of reliable estimation of these uncertainties, and suggested a rough 'envelope of uncertainty' of $1-2 \%$ (not including the systematic errors from ignoring the photonuclear component). The confidence level on this estimate is not 
given, but it is likely $95 \%$ (by extension of how NIST typically report uncertainties e.g., on the mean ionization energy ${ }^{91}$ ). In this chapter, an attempt is made to deduce a more realistic estimate of photon cross section uncertainties $(\S 6.1)$ using two different experimental data sets: the transmission data of this study ( $(6.2)$ and a collection of experimental measurements of cross sections from the literature ( $\S 6.3)$. It is useful to note beforehand that the methods used below for estimating photon cross section uncertainties are approximate. However, the results are still useful, given that the literature is lacking quantitative uncertainty analysis that is driven by direct comparisons to experimental data.

\subsection{Method of analysis}

The photon cross sections used for this analysis are the NIST XCOM compilation ${ }^{35}$ plus the IAEA photonuclear data. ${ }^{36}$ The combination of the two sets makes the most accurate cross sections available. The following hypothesis is the basis of the analysis: the shape of the XCOM + IAEA cross sections is assumed to be a close model of the true cross sections. For a given element, an energy-independent scaling factor, $\alpha_{\text {min }}$, is sought ( $\left.\S 6.3\right)$ such that it achieves the minimum RMS deviations between a large collection of experimentally-measured cross sections on one hand and $\alpha_{\min } \times(\mathrm{XCOM}+\mathrm{IAEA})$ on the other hand. A similar scaling factor is sought $(\S 6.2)$ to achieve the best fit between the measured and the EGSnrc-calculated transmission data of this study when $\alpha_{m i n} \times(\mathrm{XCOM}+$ IAEA $)$ cross sections are used in EGSnrc. In an ideal case, if XCOM + IAEA are the true cross sections, and the statistical uncertainties have been properly assessed in the experimental uncertainty budget, and the systematic errors have been either corrected for or included in the uncertainty budget, then $\alpha_{m i n}$ should be identically unity, and the corresponding reduced $\chi_{\min }^{2}$ should also be unity. In a real case, the absolute difference of $\alpha_{\text {min }}$ from unity, i.e., $\left|\alpha_{m i n}-1\right|$ (given

\subsection{METHOD OF ANALYSIS}


in per cent for all the results in this chapter), can be taken as a surrogate for the uncertainty in the XCOM + IAEA data, provided that the reduced $\chi^{2}$ is still reasonably close to unity. The assumption of a universal scaling factor may not be sufficient since the XCOM + IAEA data may have systematic errors in their shape. The potential for energy-dependent scaling is highlighted in $\S 6.4$.

The $68 \%$ confidence bounds, $u$, on $\alpha_{\min }$ are determined using a graphical method ${ }^{86}$ Briefly in this method, $\chi_{\text {lim }}^{2}$ is the limit value on $\chi_{\min }^{2}$ at a given confidence level. The absolute difference between the value of $\alpha$ that corresponds to $\chi_{\min }^{2}$ and its value that corresponds to $\chi_{\text {lim }}^{2}$ is $u$ at the confidence level stated for $\chi_{\text {lim }}^{2}$. There are different methods to determine $\chi_{\text {lim }}^{2}$. In this study, the different methods are applied, and the most conservative bounds on $\alpha_{\min }$ are used. Since $\alpha_{\min }$ is only a first-order surrogate of cross section uncertainties, it would be too strong to state that the $68 \%$ lower and upper bounds on cross section uncertainties are $\left|\alpha_{\min }-1\right|-u$ and $\left|\alpha_{\min }-1\right|+u$, respectively. Instead, assuming that $\alpha_{\min }$ follows a normal distribution, a single-sided upper bound estimate is extracted as follows: the cumulative probability of a normal distribution with zero mean and unit standard deviation shows that the integral from $-\infty$ to +1.65 is $95 \%$ of the total area. Therefore, the $95 \%$ upper bound estimate for cross section uncertainties can be taken as $\left|\alpha_{\min }-1\right|+1.65 u$.

\subsection{Estimation using transmission data}

The level of detail of the transmission measurements and calculations in this study makes it plausible to attribute the discrepancies beyond the uncertainty bars in Fig. 4.6 (p.73) to cross section uncertainties. This is used here to extract $\alpha_{\min }$ that minimizes the differences in Fig. 4.6. It would be computationally very intensive to systematically scale the attenuator cross sections by small fractions of a per cent, and repeat the EGSnrc calculations for all the beam/target/attenuator/detector combinations to sta-

\subsection{ESTIMATION USING TRANSMISSION DATA}


tistical uncertainties smaller than the effect of the cross section changes on transmission. As an alternative, a few calculations of this type are performed and compared to calculations using Eq. 1.1 (p. 8) with pre-calculated EGSnrc spectra as point sources. For a given cross section change, the calculations using Eq.1.1 yield transmission changes that agree with their respective Monte Carlo calculations to better than $0.1 \%$. Therefore, for the analysis in this section, Eq. 1.1 is used to calculate the transmission ratios that correspond to the cross section changes, and these ratios are used as a correction to the EGSnrc results.

For $\mathrm{C}$ attenuators, Fig. 6.1 shows that for the combined data of all beams [70 data points, with 69 degrees of freedom $(d o f)], \alpha_{\min }-1$ is $(+0.41 \pm 0.05) \%$, with a $\chi_{\min }^{2} / d o f$ of 0.8 . Therefore the $95 \%$ upper bound estimate of photon cross section uncertainties for $\mathrm{C}$ using the transmission data of this study is $0.41+1.65 \times 0.05=0.49 \%$, as shown in Table 6.1. Although not used in the analysis with energy-independent scaling, it is useful to note that Fig. 6.1 shows that when each MV beam is optimized individually (9 dof per beam), $\chi_{\min }^{2}$ is $\sim 0.5$, and $\alpha_{\min }-1$ values vary from $+0.25 \%$ to $+0.48 \%$, with the exception of the $10 \mathrm{MV}$ beam at $0.88 \%$.

For $\mathrm{Pb}$ attenuators, Fig. 6.2 shows that for all the beam data combined, $\alpha_{\min }-1$ is $(-0.02 \pm 0.04) \%$. The corresponding $95 \%$ upper bound estimate is $0.09 \%$ (Table 6.1). Although the overall $\chi_{\min }^{2} / d o f$ is larger than unity (i.e., 1.3), when each beam is optimized separately, the individual $\chi_{m i n}^{2} / d o f$ are $\sim 0.5$. The $\alpha_{m i n}-1$ values for the individual beams vary within $\pm 0.25 \%$. These variations around unity are the reason that the overall estimate of $0.09 \%$ may be unrealistically small.

\subsection{ESTIMATION USING TRANSMISSION DATA}




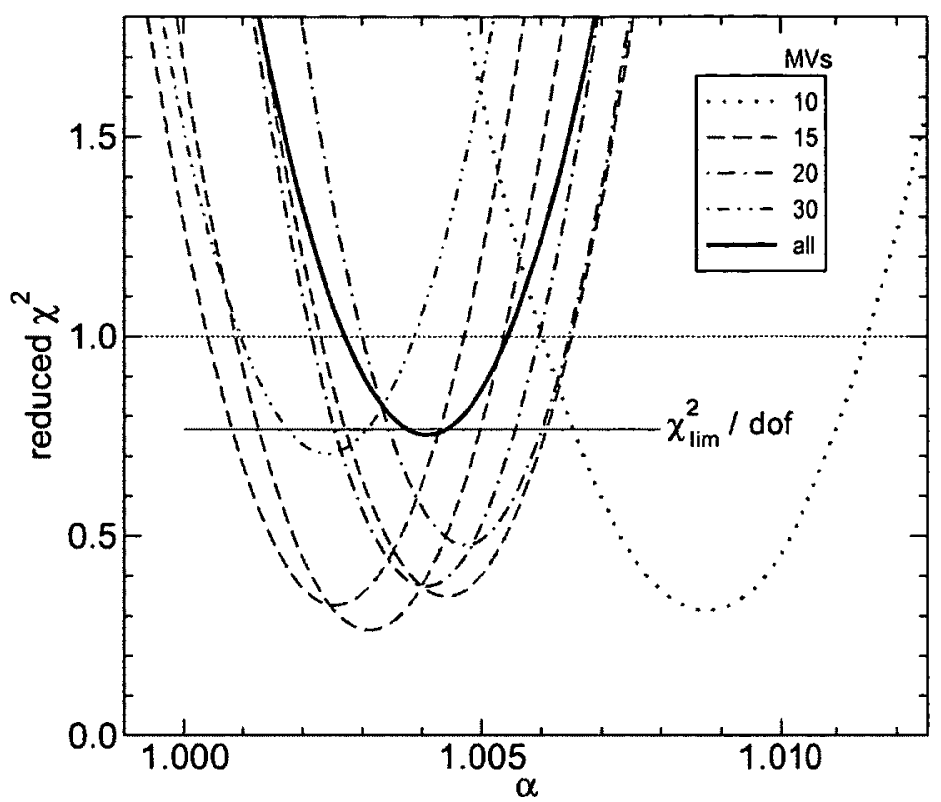

Figure 6.1: For the comparison of the measured against the EGSnrccalculated transmission data with $\mathrm{C}$ attenuators, the figure shows the variation of the reduced $\chi^{2}$ when the $C$ photon cross sections used in EGSnrc (i.e., XCOM + IAEA photonuclear data) are uniformly scaled by $\alpha$. The horizontal solid line is the $68 \%$ limit value of the reduced $\chi^{2}$, i.e., $\chi_{\lim }^{2} / d o f$, and it is used to estirnate the confidence bounds on $\alpha_{\min }$ : From Paper $\mathrm{V}$.

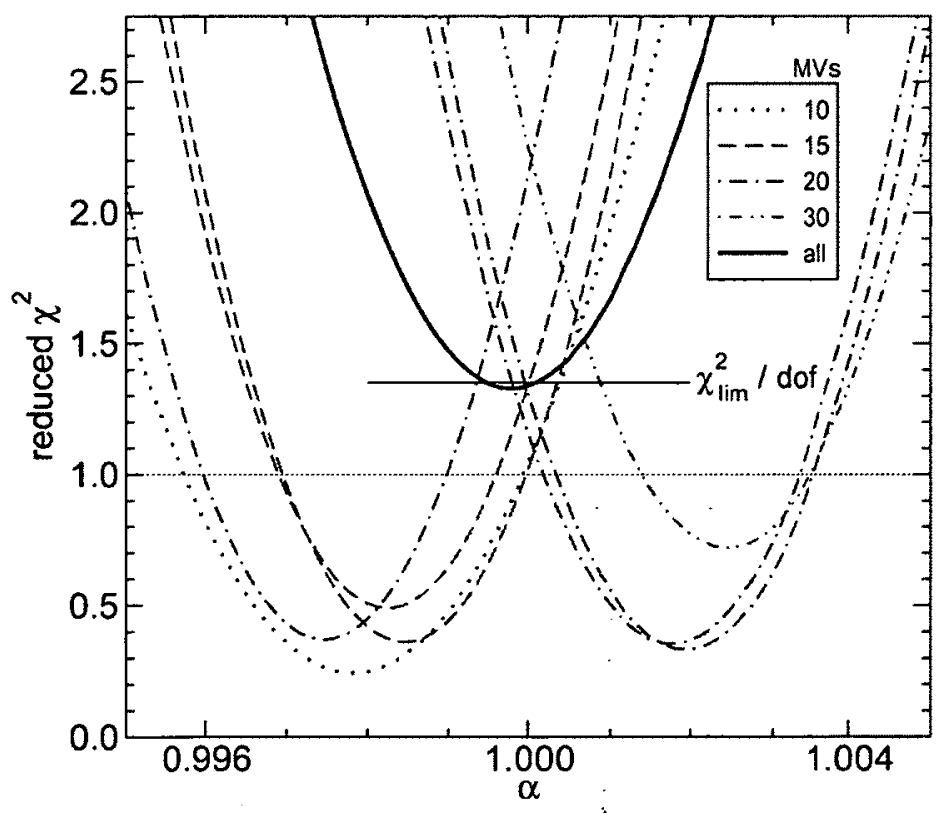

Figure 6.2: Same as figure 6.1, but for the $\mathrm{Pb}$ attenuators. From Paper V. 
Table 6.1: The 95\% upper bound estimates of photon cross section uncertainties for $\mathrm{C}$ and $\mathrm{Pb}$ using two experimental data sets and assuming energy-independent uncertainties.

\begin{tabular}{|c|ccc|ccc|cc|}
\hline & \multicolumn{3}{|c|}{ Transmission } & \multicolumn{3}{c|}{ Literature exp. } & \multicolumn{2}{c|}{ Final 95\% bounds } \\
& $95 \%$ & dof & $\chi_{\text {min }}^{2} /$ dof & $95 \%$ & dof & $\chi_{\text {min }}^{2} /$ dof & Per element & Overall \\
\hline $\mathrm{C}$ & $0.49 \%$ & 69 & 0.8 & $0.12 \%$ & 182 & 2.2 & $0.49 \%$ & \multirow{2}{*}{$0.7 \%$} \\
$\mathrm{~Pb}$ & $0.09 \%$ & 69 & 1.3 & $0.67 \%$ & 104 & 5.2 & $0.67 \%$ & \\
\hline
\end{tabular}

\subsection{Estimation using literature cross section measurements}

NIST offers a comprehensive bibliography of the publications that measured photon cross sections. Ben Spencer, a collaborator in Paper V, used this bibliography to extract the measured cross section data for $\mathrm{C}$ and $\mathrm{Pb}$. The author of this thesis compared these experimental data to $\alpha \times(\mathrm{XCOM}+\mathrm{IAEA})$ to extract $\alpha_{\text {min }}$.

The photon sources used for cross section measurements in the literature include radioactive sources, synchrotron radiation, nuclear reactions that produce gamma rays (e.g., neutron capture), and bremsstrahlung beams. The commonly used detectors are $\mathrm{NaI}(\mathrm{Tl}), \mathrm{Ge}(\mathrm{Li})$ and HPGe for direct spectroscopy, $\mathrm{Si}(\mathrm{Li})$ detectors, ion chambers, and magnetic Compton spectrometers. The common sources of uncertainties are: (a) counting statistics, small-angle scatter (coherent or incoherent); and background effects; (b) attenuator uncertainties related to the inexact mass thicknesses and the impurities; (c) radiation source uncertainties related to the exact source energy, the decay of daughter nuclei in the source, and the energy variations in the bremsstrahlung beams; and, (d) detector uncertainties related to photopeak drifts, energy calibration of the spectrometers, pulse pileup and detector dead time correction. The typical reported uncertainties on the measured cross sections are $\leq 1 \%$, and can be as large as $5 \%$. 
The analysis includes experimental data in the energy range of 0.1 to $40 \mathrm{MeV}$. The experiments included are those published no earlier than 1950 , whose reported uncertainties are $\leq 2 \%$, and their reported cross section values are within $4 \%$ of the $\mathrm{XCOM}+\mathrm{IAEA}$ values (since it is reasonable to assume that the current knowledge of photon cross sections is better than $4 \%$ ). The total number of experiments included is 21 for $\mathrm{C}$ and 28 for $\mathrm{Pb}$. The total number of data points is 183 for $\mathrm{C}$ and 105 for $\mathrm{Pb}$.

The results of the analysis are shown in Fig.6.3. For $\mathrm{C}$ and $\mathrm{Pb}$, respectively, $\left|\alpha_{\min }-1\right|$ is $(0.05 \pm 0.04) \%$ and $(0.50 \pm 0.10) \%$. The corresponding $95 \%$ upper bound estimates are, respectively, $0.12 \%$ and $0.67 \%$ (Table 6.1). The $\chi_{\min }^{2} / d o f$ for both $\mathrm{C}$ and $\mathrm{Pb}$ is large (2.2 and 5.2, respectively). There are two possible reasons for this: (a) the uncertainties in the experimental data are under-estimated; and/or, (b) the XCOM + IAEA data have systematic energy-dependent errors that make them unable to model the experimental data accurately. These two reasons are investigated by analyzing the residuals (Fig. 6.4). The spread of the data in Fig. 6.4 is larger than the stated experimental uncertainties, which suggests that some of the experimental uncertainties are under-estimated [reason (a) above]. For C, the residuals suggest that the upper bound estimate of $0.12 \%$ is very optimistic. For $\mathrm{Pb}$, there is an indication of energy dependence of the residuals (discussed in the following section), suggesting possible deficiencies in the XCOM + IAEA data [reason (b) above].

The photonuclear component becomes relevant for $\mathrm{C}$ at energies above $\sim 17 \mathrm{MeV}$. When the $\alpha_{\min }$ analysis for $\mathrm{C}$ is repeated up to only $17 \mathrm{MeV}$ using the XCOM data alone, the results are found to be very comparable to those shown above with literature experimental data up to $40 \mathrm{MeV}$. This indicates that while the IAEA photonuclear data account for a systematic component that cannot be ignored, their presence does not dominate the outcome of the analysis. This test is not as informative for $\mathrm{Pb}$ because the photonuclear component becomes relevant at much lower energies $(\sim 8 \mathrm{MeV})$.

\subsection{ESTIMATION USING LITERATURE CROSS SECTION MEASUREMENTS}




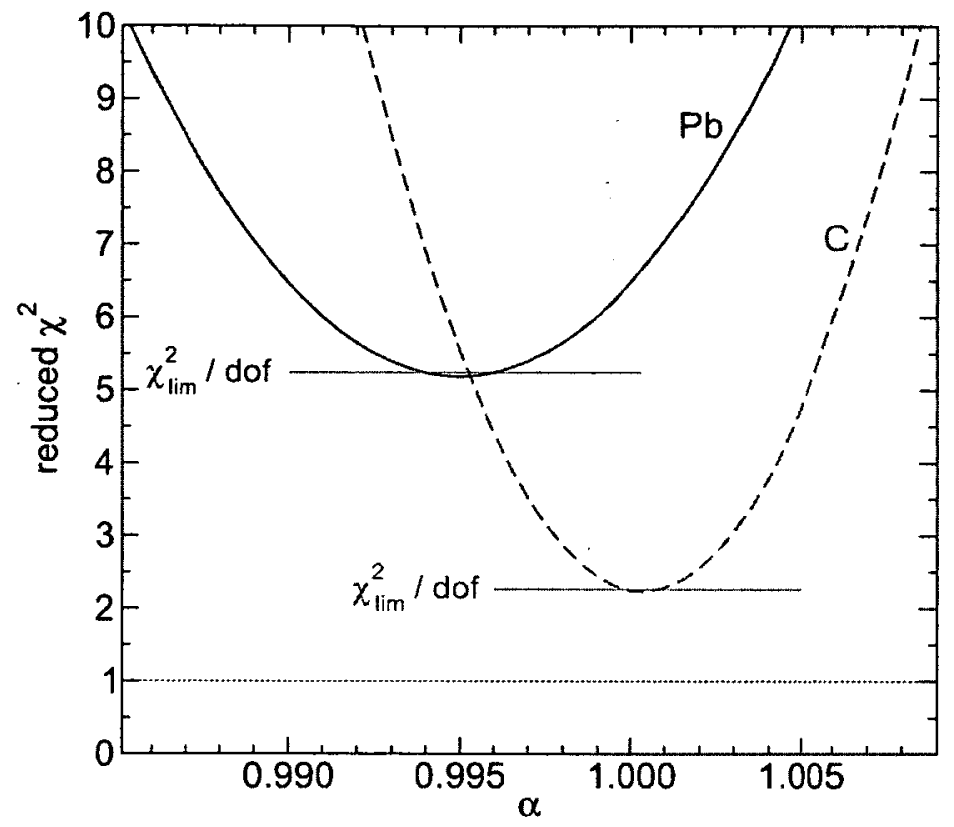

Figure 6.3: For the comparison of the photon cross section measurements from the literature against the XCOM + IAEA photonuclear data, the figure shows the variation of the reduced $\chi^{2}$ when the XCOM + IAEA photonuclear cross sections are uniformly scaled by $\alpha$. The horizontal solid lines are the $68 \%$ limit values of the reduced $\chi^{2}$, i.e., $\chi_{\text {lim }}^{2} / d o f$, and they are used to estimate the confidence bounds on their respective $\alpha_{\text {min }}$. From Paper V.

\subsection{Discussion}

Given the approximate nature of the uncertainty analysis above, the final upper bound estimate for a given element is taken as the larger of the two bounds estimated using the two experimental data sets. Thus the final bounds are $0.49 \%$ for $\mathrm{C}$ and $0.67 \%$ for $\mathrm{Pb}$. Similarly, an overall material-independent energy-independent $95 \%$ upper bound estimate on photon cross section uncertainties can be taken as $0.7 \%$ (Table 6.1).

Energy-dependent errors are plausible because of the change with energy for the relative contribution of different physics processes and the errors associated with their models. For the $\mathrm{C}$ data in Fig. 6.1, the individual $\alpha_{\min }-1$ values do not exhibit energy dependence versus the fluence-weighted mean energies of the spectra of their 


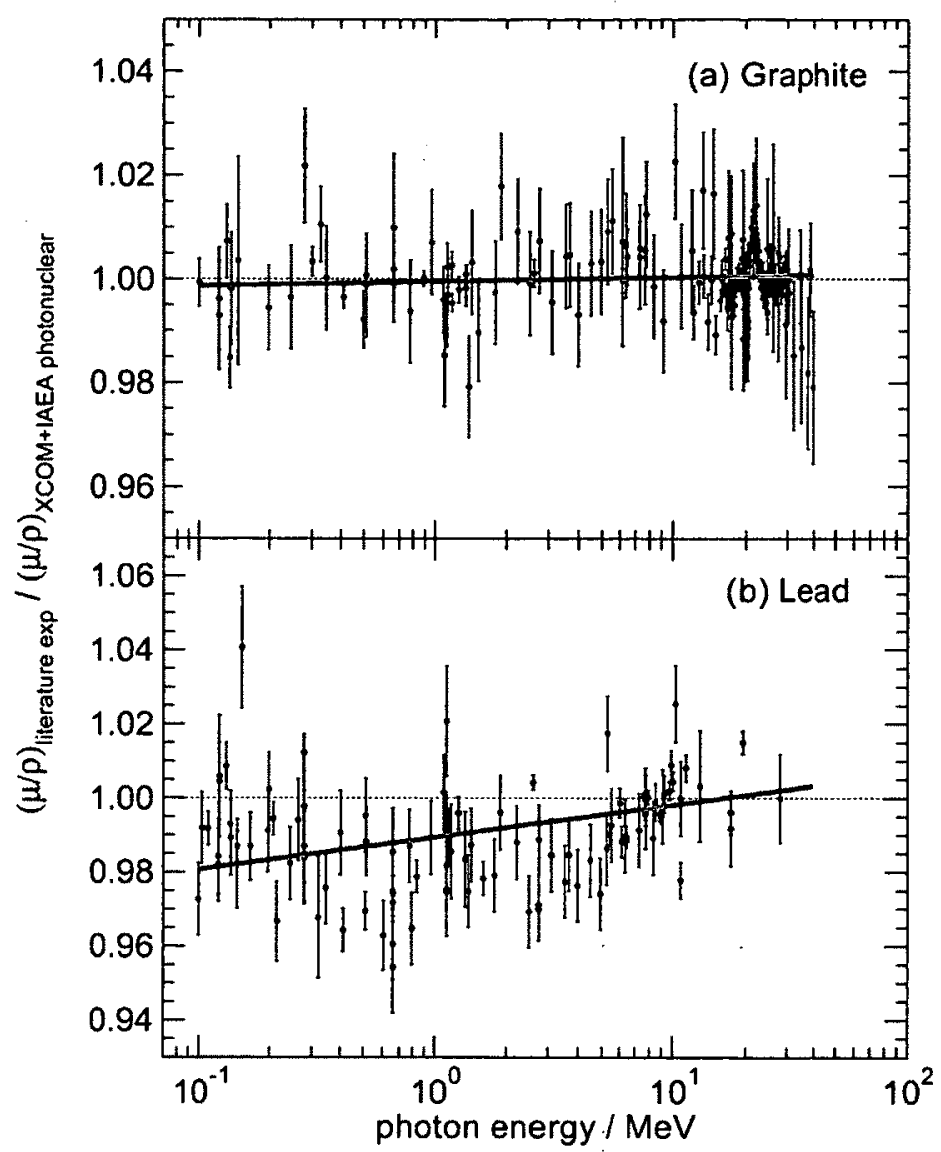

Figure 6.4: Ratios of the photon cross sections measured in the literature to the $\mathrm{XCOM}+$ IAEA photonuclear data for $\mathrm{C}$ and $\mathrm{Pb}$. The solid lines are linear fits of the ratios, weighted by the variances on the ratios, versus the logarithm of the photon energy. From Paper V.

beams. Similarly, the $\mathrm{C}$ data in Fig. 6.4 indicate negligible energy dependence. For the $\mathrm{Pb}$ data in Fig. 6.2, the individual $\alpha_{m i n}-1$ values show positive correlation with the fluence-weighted mean energies. The variation is $\sim 0.5 \%$ between $1.5 \mathrm{MeV}(10 \mathrm{MV})$ and $5.3 \mathrm{MeV}(30 \mathrm{MV})$. When a linear model of this variation is applied to the XCOM + IAEA data, the $\chi_{m i n}^{2} / d o f$ worsens to 2.3 , compared with 1.3 for the energy-independent scaling. This is because the extrapolation of the model to the full energy range is not likely accurate, particularly that it is based on mean energies of spectra where real energy-dependence of the uncertainties can be averaged out. The $\mathrm{Pb}$ data in Fig. 6.4 
also indicate energy dependence with positive correlation. The variation is $\sim 2.5 \%$ over the full energy range. Applying a linear model of this variation to the XCOM + IAEA data improves $\chi_{m i n}^{2} / d o f$ from 5.2 to 3.9 , but not to unity. Taking the $2.5 \%$ variation as the $95 \%$ upper bound estimate would be overly conservative, given the large scatter of the data, and given the level of agreement of the calculated and measured transmission data for $\mathrm{Pb}$ attenuators in this study (Fig. 4.6, p.73). Photoelectric cross sections are affected by a debatable re-normalization factor which, if applied, would lower the XCOM values by a few per cent. ${ }^{35}$ The photonuclear cross sections have large uncertainties. These two observations suggest that, compared with $\mathrm{C}$, the larger likelihood of energy-dependent errors for $\mathrm{Pb}$ could be related, in part, to the increased importance of the photoelectric component (which still makes $25 \%$ of the total cross section at $1 \mathrm{MeV}$ ) and, in part, to the relevance of the photonuclear component at much lower energies $(\sim 8 \mathrm{MeV})$.

\subsection{Summary}

In this chapter, the experimental and computational transmission data of this study, as well as the experimental measurements of photon cross sections in the literature, are used to deduce an estimate of photon cross section uncertainties that is more realistic than the currently used 'envelope of uncertainty' of $1-2 \%$. For the energy range from $0.1-40 \mathrm{MeV}$, the overall material-independent energy-independent $95 \%$ upper bound estimate is $0.7 \%$. The results using the transmission data of this study are more consistent, given that their $\chi_{\min }^{2} / d o f$ are much closer to unity. Indications are found for plausible energy-dependent errors for $\mathrm{Pb}$, although a firm conclusion is difficult to make because of many complicating factors of the analysis. Although the methods used are

approximate, the results are still useful, given that the literature is lacking quantitative uncertainty analysis that is driven by direct comparisons to experimental data. 


\section{Chapter 7}

\section{Summary and conclusions}

In this study, a physics-based approach was proposed for the ill-posed problem of unfolding linac photon spectra from transmission measurements. The approach was independently validated and then successfully demonstrated on a typical clinical linac. This closing chapter starts by recalling the list of the limitations of previous studies from $\S 1.7$, and highlighting how they were addressed in this study ( $\S 7.1$ ). Based on the experimental issues encountered, recommendations are given for accurate transmission measurements $(\S 7.2)$. The overall conclusions are given in $\S 7.3$. The closing sections provide a list of possible extensions of this work $(\$ 7.4)$, and a list of potential applications ( $(7.5)$.

\subsection{Summary of how previous limitations were addressed}

This section reproduces the list of limitations of previous studies from $§ 1.7$ and highlights how they were addressed in this work.

- Lack of direct independent validation: This issue was addressed by performing the validation measurements on a dedicated research linac whose photon spectra were previously measured using a NaI detector, and whose electron beam parameters are accurately and independently known. The MV range of the beams used (10 - $30 \mathrm{MV})$ provided a particularly rigorous validation because accurate spectral unfolding is most 
challenging in this range. Validation results of the proposed approach show that the energy fluence spectra can be unfolded with RMS energy fluence deviations of $4.5 \%$, and the incident electron energies can be unfolded with an accuracy of $\sim 3 \%$.

- Limited energy differentiation: This was addressed by introducing the concept of combining transmission data measured for multiple attenuators of very different atomic numbers using multiple detectors of opposite energy response. Different measurement configurations were computationally tested, and the configuration with the highest energy sensitivity was chosen.

- Inability to unfold the maximum energy: In this study, the maximum photon energy was unfolded as a free parameter. This is made possible by the improvement of the energy differentiation in the input transmission signals using multiple attenuators and detectors, and by the improvement of the robustness of the minimization using a spectral functional form with a limited number of free parameters.

- Systematic errors in the system kernel: Kernel errors were addressed in multiple ways. The detector energy response was calculated using Monte Carlo and validated experimentally to avoid introducing Monte Carlo bias in the unfolded quantities. Monte Carlo was also used to correct the measured transmission signals for non-ideal attenuation conditions that are difficult to eliminate or correct for experimentally. The photonuclear component was added to the cross sections used in the system kernel.

- Limited robustness: Robustness was significantly improved by using a new physicsbased functional form with only four free parameters. The function outperformed existing functions in terms of the combination of accuracy, flexibility and robustness. The function was able to fit a diverse set of spectra with RMS energy fluence deviations of $1.7 \%$, and to fit the maximum energy within $1.4 \%$. The use of the functional form eliminated the need for sophisticated unfolding algorithms (with their attendant issues).

\subsection{SUMMARY OF HOW PREVIOUS LIMITATIONS WERE ADDRESSED}


- Limited experimental accuracy: This was addressed by experimentally investigating a large number of influence quantities and developing reliable methods to correct for them. Auxiliary Monte Carlo was used to confirm and/or supplement experimental sensitivity studies. Detailed experimental uncertainty budgets were used, which allowed for extracting meaningful confidence bounds on the unfolded quantities. The resulting experimental protocol allowed for a measurement accuracy of $\sim 0.4 \%$ on the smallest transmission signals over a signal range of a factor of $\sim 60$.

- Lack of a comprehensive study: In this work, improvements in the physics, numerical and experimental aspects of the problem were proposed. Except for the functional form, the improvements are independent of the unfolding details and would thus improve the relative performance of any future unfolding algorithm. It is hoped that this work contributes to filling the void of lacking a comprehensive study on the subject.

\subsection{Recommendations for accurate transmission measurements}

The following recommendations are made for accurate transmission measurements, down to transmission values of $\sim 1.7 \%$. Reference class $0.6 \mathrm{cc}$ Farmer chambers present a reasonable compromise between reliability, signal size and narrow-beam geometry. Their response can be easily manipulated with the choice of the buildup cap material. Equilibration at the start of the irradiation and when reversing the polarity is important. Alignment and drifts in the beam direction should be closely checked at the detector location because the narrow-beam geometry amplifies their effects. Reliable corrections for linac output fluctuations and linac drifts can be achieved with the combined use of the monitor signal, the field-to-monitor ratio, and the Cu-to-monitor ratio. A reasonable number of randomized short- and medium-term repeats is necessary to reduce bias from slow linac drifts. Reasonably-short signal collection times help identify 
and eliminate the data acquired during short-term beam instabilities. Automating the measurements provides obvious convenience, but it also facilitates more randomized repeats and reduces beam instability effects because of the reduced beam interruption. Polarity corrections are large and variable, therefore they should be measured individually for each data set; simple precautions such as irradiating the chamber side-on (not head-on) or adjusting cable positions help reduce the magnitude and the uncertainty on polarity correction. Ion recombination is not a major correction for the typical dose rate variations in transmission measurements. Leakage varies significantly depending on the chamber-cable-electrometer system used. Therefore leakage contribution should be characterized for the individual system components, and reduced if necessary (e.g., larger chamber, shorter cables and/or different electrometer). If the electrometer has to be moved into the radiation room (as done in this study), it should be shielded to protect its radiation-sensitive circuitry without blocking proper heat exchange. Room scatter can be quantified using the shadow-cone technique. Apparatus scatter should be quantified experimentally and/or by Monte Carlo simulations. Accurate knowledge of the mass thickness of the attenuators is critical. The mass thickness can be determined accurately using a combination of linear dimensions, volume measurements, radiation measurements, CT scans, and Monte Carlo sensitivity studies. High-purity attenuators are not essential, but they reduce the uncertainty due to the inexact knowledge of the impurities. Temperature should be individually monitored at the different locations where signals are acquired, and corrected for if necessary. All the corrections above should remain at the level of small perturbations to the main transmission signals in order for the fully-corrected signals to be credible. Monte Carlo simulations are a useful tool for systematic investigation of influence quantities to confirm and/or supplement experimental sensitivity studies. 
The fully-corrected transmission signals still include unavoidable components from non-primary radiation. If the purpose of a study is to benchmark a Monte Carlo code, then these effects do not need to be corrected for because they are included in both the measured and the calculated transmission data. However, if the purpose of the study is to obtain a pure primary transmission signal to be used for, e.g., spectral unfolding, then one of the two methods of $\S 2.7$ to correct for non-ideal attenuation conditions should be applied to the fully-corrected experimental signals.

\subsection{Conclusions}

The main conclusion of this study is that clinical linac photon spectra and their respective incident electron energies can be unfolded reliably and accurately using the transmission analysis approach, provided that the full potential of the approach is used, and that the due rigor is taken on both its computational and experimental components. The approach proposed in this study allows for unfolding the energy fluence spectra with RMS energy fluence deviations of $4.5 \%$, and the incident electron energies with an accuracy of $\sim 3 \%$. The unfolding does not require knowledge of the linac head details or a-priori knowledge of the electron energy. Although the lowest-MV beam used for the validation on the research linac is $10 \mathrm{MV}$ (the lowest stable beam), the method should be applicable down to $6 \mathrm{MV}$ and to the MV imaging beams because the energy differentiation is even better for these lower-MV beams. Similarly, the beams used for the validation are flattening-filter free, but the results are equally applicable to beams with flattening filters. The practicality of the validated approach is successfully demonstrated on a typical clinical linac for 6, 10, and $25 \mathrm{MV}$ beams.

A truly flexible spectral functional from is found to require at least four free parameters, one of which is the maximum energy. The specific function proposed is physics-based, accurate, flexible, and not over-parameterized. These attributes make 
it play a central role in taming the unfolding problem. Similarly, the accuracy and robustness are improved when using the simple (but surprisingly unexploited) idea of improving energy differentiation by combining transmission data for multiple detectors of opposite energy response and multiple attenuators of very different atomic numbers.

Computational and experimental influence quantities have a major effect on the accuracy of the unfolded quantities. On the computational side, the accuracy of the detector energy response model is the most significant component. Other components are the photonuclear effect and non-ideal attenuation conditions. On the experimental side, the dominant potential contributors to measurement uncertainties are beam drifts, polarity effects, leakage and attenuator mass thickness. Ignoring these influence quantities can introduce errors of more than $10 \%$ in the measured transmission signals, and the effects are most significant for the smallest signals. The experimental protocol developed in this study allows for a measurement uncertainty of $\sim 0.4 \%$ (not including electron beam uncertainties) over a signal range of a factor of $\sim 60$. With the challenges associated with smaller transmission signals, a useful observation is that accurate spectral unfolding can be achieved with a transmission cutoff of only $10 \%$, provided that the other components of the proposed approach are implemented.

A unique aspect of this study is its hybrid experimental/Monte Carlo nature, which was a catalyst for refinements on both sides. On one hand, auxiliary Monte Carlo calculations were used to refine the experimental setup and to confirm and/or supplement experimental sensitivity studies. On the other hand, disagreements between measurements and EGSnrc calculations at earlier stages of the project prompted the implementation of photonuclear attenuation, and the re-engineering of the XCOM cross sections in EGSnrc. Accurate transmission calculations require the use of the most accurate cross section data and physics models available in EGSnrc, particularly the more accurate bremsstrahlung angular sampling option (up to a $6.4 \%$ effect) and the modelling of

\subsection{CONCLUSIONS}


photonuclear attenuation (up to a $5.6 \%$ effect). Measured transmission data on the research linac are used as a sensitive benchmark of EGSnrc. Results indicate that EGSnrc is accurate within $0.2 \%$ for relative ion chamber response calculations. The full data sets needed to benchmark other Monte Carlo codes are available in Report III.

Not surprisingly, photon cross section uncertainties are found to be the ultimate limiting factor in transmission analysis. An attempt is made to use the experimental and computational transmission data of this study, as well as the experimental measurements of photon cross sections in the literature, to deduce an estimate of photon cross section uncertainties. The overall material-independent energy-independent $95 \%$ upper bound estimate is $0.7 \%$. The agreement between measured and calculated transmission data suggest that this estimate is more realistic than the currently used 'envelope of uncertainty' of $1-2 \%$. Indications are found for plausible energy-dependent errors, but a firm conclusion is difficult to make. Although the methods used in this study for estimating photon cross section uncertainties are approximate, the results are still useful, given that the literature is lacking quantitative uncertainty analysis that is driven by direct comparisons to experimental data.

\subsection{Future work}

The following is a list of a few possible extensions to the work presented in this study.

- Extending the concept of using detectors of different energy response to physically different detectors. This can lead to further improvements in energy differentiation.

- Off-axis measurements on both the research and the clinical linacs. For the research linac, off-axis $\mathrm{NaI}$ measurements are available for a $15 \mathrm{MV}$ beam with $\mathrm{Be}, \mathrm{Al}$ and $\mathrm{Pb}$ targets. ${ }^{12}$ A different 'rotational' experimental setup would be needed, and alignment issues will need to be addressed carefully. 
- Transmission measurements on linacs from different manufacturers for the purpose of building a library of 'measured' clinical spectra.

- Bin-by-bin iterative spectral unfolding using all the improvements in this work, except for the functional form. The same applies to exploring new unfolding algorithms.

- Extending the implementation of photonuclear interactions in EGSnrc to model the secondaries. This will not have an effect on transmission calculations, but it will find its applications as a useful simulation tool.

- Systematic analysis of photon cross section uncertainties for all elements rather than only $\mathrm{C}$ and $\mathrm{Pb}$, to help extract more concrete estimates of those uncertainties.

- Using the functional form to extract spectra from depth-dose measurements. Experimental and computational aspects of that approach were investigated in parallel with the work presented in this thesis. Results show that, while the measurements are much simpler than they are for transmission, accurate and robust unfolding is much more difficult because of the reduced energy differentiation, the electron contamination, the field-size dependence, and the phantom scatter. This work will be presented elsewhere. $^{23}$

\subsection{Potential applications}

The following is a list of a few potential applications of the work presented in this study.

- A validated cost-effective tool to determine the photon spectra of clinical photon beams during beam commissioning, re-commissioning, or extended quality assurance. A variant of this application was done during the course of this study when the klystron had to be replaced, where the matching of the measured transmission signals before and after replacing the klystron was a useful part of the research linac re-commissioning. 
- Investigating the spectral differences between dosimetrically-matched linacs from different manufacturers. This knowledge is useful for consistency in clinical trials and for better understanding and use of dosimetrically-matched beams. Similarly, spectral differences between dedicated IMRT machines (e.g., Tomotherapy and Cyberknife) would be insightful. A collaboration on these investigations is already arranged.

- Realistic modelling of beam softening off-axis (especially for beams with flattening filters), which is particularly useful for modelling the detector response off-axis. This is achievable by using off-axis transmission measurements to unfold the corresponding spectra, and can be extended to other clinical situations with relevant spectral changes.

- The functional form on its own is of general utility to describe photon spectra, - e.g., in virtual source modelling.

- Benchmarking of Monte Carlo codes other than EGSnrc using the detailed high accuracy transmission measurements of this study in combination with the complete description of the setup in Report III.

- Re-analysis of the effects of cross section uncertainties on fundamental dosimetric quantities (e.g., beam quality conversion factors and air-kerma standards) in light of the more realistic estimates of cross section uncertainties, as presented in this study.

\subsection{POTENTIAL APPLICATIONS}




\section{Appendix A}

\section{Benchmark set of spectra}

This appendix describes a benchmark set of 65 spectra of clinical and research linacs. The set is generated to test the accuracy and flexibility of different functional forms (§2.1 and App.B). Subsets of these spectra are used throughout this study for different investigations. The set includes beams from $3.5 \mathrm{MV}$ to $30 \mathrm{MV}$. The clinical spectra are for the Varian, Elekta, Siemens, Tomotherapy and Cyberknife linacs. The research spectra are for the linacs of the National Research Council of Canada (NRC) and the National Physics Laboratory (NPL) in the UK. The set includes spectra on- and offaxis, with a flattening filter (WFF), flattening-filter free (FFF), and in treatment and imaging modes. Spectra are generated by Monte Carlo using beam models that were validated in other studies. The most useful aspect of the set is its wide variety of realistic spectral shapes with high energy resolution and high statistical precision.

Varian, Elekta and Siemens spectra: BEAMnrc ${ }^{37,38}$ is used to model nine linac beams [Varian Clinac $4 \mathrm{MV}$ and Clinac $2100 \mathrm{C} / 2300 \mathrm{C}$ 6, 10, 15, and $18 \mathrm{MV}$; Elekta SL25 6 and $25 \mathrm{MV}$; and Siemens KD 6 and $18 \mathrm{MV}$ ]. The proprietary specifications of the linac heads and the electron parameters are taken from the original study of SheikhBagheri and Rogers. ${ }^{57}$ Simulations are done WFF and FFF. Within a $40 \times 40 \mathrm{~cm}^{2}$ field at $100 \mathrm{~cm}$ SSD (source-to-surface distance), on- and off-axis spectra are extracted in, respectively, a $100 \mathrm{~cm}^{2}$ central area and an annulus between radii of 15 and $20 \mathrm{~cm}$. Each 
spectrum has 100 equal-size energy bins (bin widths range from $40 \mathrm{keV}$ to $200 \mathrm{keV}$ ). In total, 36 spectra are generated (nine beams, WFF, FFF, on- and off-axis).

NRC Vickers linac spectra: FLURZnrc ${ }^{40}$ is used to model the setup of Faddegon et $a l^{6,11,12}$ for the measurement of forward bremsstrahlung production from thick targets. The beams are $10-30 \mathrm{MV}$ in $5 \mathrm{MV}$ increments, and the targets are $\mathrm{Be}, \mathrm{Al}$ and $\mathrm{Pb}$. The details of geometry, materials and electron parameters are taken from Faddegon et al. ${ }^{6}$ Central-axis spectra are generated within a $0.5^{\circ}$ cone half angle. Each spectrum has between 55 and 110 energy bins of variable size to match those of the measured spectra. In total, 11 central-axis spectra are generated: one $15 \mathrm{MV}$ spectrum with a $\mathrm{Be}$ target and five MV spectra for each of the $\mathrm{Al}$ and $\mathrm{Pb}$ targets.

NPL linac spectra: BEAMnrc is used to model the NPL linac beams using the details from the original study of Walters and Rogers. ${ }^{92}$ The beams modelled are $4,6,8,10$, 12, 16 and 19 MV. Each beam has two configurations: WFF alone ('light' filtration) and WFF plus $5-14 \mathrm{~cm}$ of additional Al filtration ('heavy' filtration). Spectra are extracted from $\sim 105 \mathrm{~cm}^{2}$ central field at $\sim 119 \mathrm{~cm}$ SSD. In total, 14 spectra, each with 100 equal-size energy bins, are generated.

Tomotherapy spectra: Jeraj et al ${ }^{93}$ used MCNP4 $43^{94}$ to model a Tomotherapy unit using its proprietary specifications. The benchmark set includes three of those spectra (provided by R. Jeraj): two treatment spectra averaged over $4 \times 5 \mathrm{~cm}^{2}$ areas at $85 \mathrm{~cm}$ SSD and centered at, respectively, $2.5 \mathrm{~cm}$ and $17.5 \mathrm{~cm}$ off axis; and, one imaging spectrum averaged over a central area of $4 \times 40 \mathrm{~cm}^{2}$ at the same SSD. The spectra have between 40 and 70 equal-size energy bins.

Cyberknife spectrum: Araki ${ }^{95}$ used EGSnrc to model a Cyberknife unit. The set includes the central-axis spectrum (provided by $\mathrm{F}$. Araki), which is averaged over a 6-cm-diameter area at $80 \mathrm{~cm} \mathrm{SSD}$ and has 140 equal-size energy bins. 


\section{Appendix B}

\section{Previous functional forms}

In this appendix, 11 functional forms that were used in the literature in various contexts to specify photon spectra are described, and their accuracy and flexibility is quantified using a benchmark set of 65 spectra (described in App. A). Notation is standardized as defined in Table B.1. A summary of the 11 functions is given in Table B.2, along with the function proposed in this study from $\S 2.1$. For brevity, functions are called henceforth by their index (first column in Table B.2). The maximum photon energy, $E_{m}$, is treated as a free parameter for the functions that contain it, even if the original authors did not intend it as such - hence a three-parameter function implies $C_{1}, C_{2}$ and $E_{m}$. This is done because $E_{m}$ (or equivalently $E_{e}$ ) is not known for typical clinical photon beams, and fixing it to an assumed value can be a gross approximation. Some functions are strictly empirical without physics grounds: functions 2, 6, 7, 8, 10 and 11 are ansatzes. Other functions are based on physics formalisms: functions 1 and 9 are based on the Kramers' spectrum, ${ }^{96}$ functions 4 and 5 are based on the Schiff thintarget spectra, ${ }^{8,24,25}$ and function 3 and the like are based on thick-target spectra. The following is a summary of the relevant features of each function in Table B.2. Paper I contains more details.

In function $1,\left(E_{m}-E\right)$ is the Kramers' spectrum, which ignores electron scattering and breaks down at relativistic energies, thus the authors added exponential factor to 
Table B.1: Standardized notation used for the equations of the different spectral functional forms (top part), and for the metrics to evaluate the performance of those functions (bottom part). From Paper I.

\begin{tabular}{|c|c|}
\hline Symbol & Definition \\
\hline$E$ & Photon energy. \\
\hline$\psi(E)$ & Differential energy fluence at $E$. \\
\hline$n_{b}$ & Number of energy bins in a spectrum; $n_{b}=100$ \\
\hline$\psi_{b}$ & Differential energy fluence for energy bin $b$ of width $d E_{b}$. \\
\hline$\psi_{a v}$ & Average energy fluence; $\psi_{a v}=\sum_{b=1}^{n_{b}} \psi_{b} d E_{b} / \sum_{b=1}^{n_{b}} d E_{b}$ \\
\hline$E_{l}, E_{m}$ & Respectively, lowest and maximum photon energy in $\psi(E)$. \\
\hline$E_{e}$ & Mean incident electron kinetic energy. \\
\hline$E_{t}$ & Mean incident electron total energy; $E_{t}=E_{e}+m_{e} c^{2}$ \\
\hline$E_{m p}$ & Most probable energy of $\psi(E)$ for the bremsstrahlung continuum \\
\hline & (i.e., excluding the $511 \mathrm{keV}$ annihilation peak if present). \\
\hline$E_{a v}$ & Average energy of the spectrum. \\
\hline$n_{p}$ & Number of free pararneters in a functional form \\
\hline & (including $E_{m}$ or $E_{e}$ if a function has either of them). \\
\hline$C_{f}$ & Free parameter $f$ \\
\hline$\left(d \sigma_{b r} / d E\right)_{\left(x, E_{\partial}^{\prime}\right)}$ & Differential bremsstrahlung cross-section for electrons of kinetic \\
\hline & energy $E_{e}^{\prime}$ (mean kinetic energy at depth $x$ in the target). \\
\hline & An arbitrary depth in the bremsstrahlung target. \\
\hline & Atomic number. \\
\hline$\mu_{X}(E)$ & Mass attenuation coefficient of material $X$ at energy $E$. \\
\hline $\begin{array}{r}\delta\left(E-E_{511}\right) \\
d E_{511}\end{array}$ & $\begin{array}{l}\text { Dirac delta function at the center of the } 511 \mathrm{keV} \text { energy bin. } \\
\text { width of the } 511 \mathrm{keV} \text { energy bin (user-defined). }\end{array}$ \\
\hline$\% \Delta^{s}(X)$ & For spectrum $s$, the per cent deviation of fit, $f$, from truth, $t$ : \\
\hline & $\begin{array}{l}\text { For } X=\psi, \% \Delta^{s}(X)=\left(100 / \psi_{a v}^{s, t}\right) \sqrt{\left(1 / n_{b}\right) \sum_{b=1}^{n_{b}}\left(\psi_{b}^{s, f}-\psi_{b}^{s, t}\right)^{2}} \\
\text { For } X=E_{e}, E_{m}, E_{m p} \text { or } E_{a v}, \% \Delta^{s}(X)=100\left|X^{s, f} / X^{s, t}-1\right|\end{array}$ \\
\hline & Total number of spectra in the benchmark set; $n_{s}=65$ \\
\hline$\% \Delta(X)$ & $\begin{array}{l}\text { Overall per cent deviation over the } n_{s} \text { spectra for quantity } X \text { : } \\
=\left(1 / n_{s}\right) \sum_{s=1}^{n_{s}} \% \Delta^{s}(X) \text { for } X=\psi, E_{e}, E_{m}, E_{m p} \text { or } E_{a v}\end{array}$ \\
\hline
\end{tabular}

Note: The maximum photon energy, $E_{m}$, can be larger than the mean incident electron kinetic energy, $E_{e}$, because of the energy spread of the incident electron beam in typical clinical linacs. Therefore the fitted endpoint energy is compared with both the true $E_{e}$ and the true $E_{m}$. 
Table B.2: Chronological list of the functional forms analyzed in this study. Notation is given in Table B.1. Energies are in $\mathrm{MeV}$. Equation numbers from the original studies are listed for ease of cross referencing. From Paper $\mathrm{I}$.

\begin{tabular}{|c|c|c|c|c|}
\hline Index & Author(s) & $\begin{array}{l}\text { Original } \\
\text { Eq. \#(s) }\end{array}$ & $n_{p}$ & Function \\
\hline 1 & Dance and Baggerly ${ }^{97}$ & p. 38 & 2 & $\psi(E)=\left(E_{m}-E\right) \exp \left(-C_{1} E / E_{m}\right)$ \\
\hline 2 & Tarasko et al $l^{98}$ & 2 & 6 & $\psi(E)=\left(C_{1}+C_{2} E_{m}\right)\left(E / E_{m}\right)^{C_{3}+C_{4} E_{m}}\left(1-E / E_{m}\right)^{C_{5}}$ \\
\hline 3 & Ahnesjö and Andreo ${ }^{20}$ & 4 & 4 & $\psi(E)=E \exp \left(-\mu_{C_{2}}(E) C_{3}\right) \int_{0}^{C_{1}} \frac{d \sigma_{b r}}{d E} \mid \exp _{\left(x, E^{\prime}\right)}\left[-\mu_{W}(E)\left(C_{1}-x\right)\right] d x$ \\
\hline 4 & Baker; 99 Baker et al ${ }^{65}$ & 5 & 4 & $\begin{array}{c}\psi(E)=\left[\left(1-\frac{E}{E_{t}}\right)(\ln \eta-1)+C_{2}\left(\frac{E}{E_{t}}\right)^{2}(\ln \eta-0.5)\right] \exp \left(-\mu_{W}(E) C_{1}-C_{3} / E\right) \\
\eta=\left[\left(\frac{m_{n} c^{2} E}{2 E_{t}\left(E_{t}-E\right)}\right)^{2}+\left(\frac{\sqrt[3]{Z}}{111}\right)^{2}\right]^{-0.5}\end{array}$ \\
\hline 5 & Krmar et al ${ }^{71}$ & 8 & 4 & $\psi(E)=$ equation $3 \mathrm{BS}(\mathrm{e})$ in Koch and $\operatorname{Motz}^{24} \times \exp \left(-C_{1} / E^{C_{2}}-C_{3} E\right)$ \\
\hline 6 & Bloch and $\mathrm{McDonough}{ }^{67}$ & $5 \mathrm{a}, \mathrm{b}$ & 2 & $\psi(E)=\frac{E}{C_{1} C_{2}}$ for $E \leq C_{1}, \quad$ and $\quad \psi(E)=\frac{1}{C_{2}} \exp \left(\frac{-\left(E-C_{1}\right)^{2}}{2 C_{2}^{2}}\right)$ for $E \geq C_{1}$ \\
\hline 7 & Fippel ${ }^{100}$ & 11 & 3 & $\psi(E)=E^{C_{1}} \exp \left(-C_{2} E\right)$ for $\quad E_{l} \leq E \leq E_{m}, \quad$ and $\quad \psi(E)=0 \quad$ elsewhere. \\
\hline 8 & Sawchuk $^{101}$ & 4 & 2 & $\psi(E)=\sin \left[\pi\left(E-E_{l}\right) /\left(E_{m}-E_{l}\right)\right] \exp \left[-C_{1}\left(E-E_{l}\right)\right]$ \\
\hline 9 & Hinson and Bourland ${ }^{49}$ & 13 & 2 & $\psi(E)=\left(E_{m}-E\right) \exp \left(-\mu_{C u}(E) C_{1}\right)$ \\
\hline 10 & Sikora et al ${ }^{102}$ & $1-4$ & 4 & $\begin{array}{c}\psi(E)=\left(1-e^{-C_{1} E}\right)\left(e^{-C_{2} E}-e^{-C_{2} E_{m}}\right) \exp \left[-\left(\mu_{S T}\left(E_{m}\right)-\mu_{S T}(E)\right) C_{3}\right] \\
\mu_{S T}(E)=0.027(E+0.16)^{-1.2}+0.03 . \mathrm{ST} \text { is for steel. }\end{array}$ \\
\hline 11 & Davidson et $a l^{103}$ & 1 & 5 & $\psi(E)=E\left[\frac{\sqrt{\frac{E-C_{2}}{C_{3}}}+\sqrt{\frac{C_{3}}{E-C_{2}}}}{C_{1}\left(E-C_{2}\right)}\right] \exp \left[-\frac{\left(\sqrt{\frac{E-C_{2}}{C_{3}}}-\sqrt{\frac{C_{3}}{E-C_{2}}}\right)^{2}}{2 C_{1}^{2}}\right] /\left[1+\exp \left(\frac{E-C_{4}}{C_{5}}\right)\right]$ \\
\hline & & & & $C_{1}>0, \quad C_{2}<E_{l}, C_{3}>0$ \\
\hline 12 & this study & - & 4 & $\begin{array}{l}\psi(E)=\psi_{\text {thin }}(E) \exp \left(-\mu_{W}(E) C_{1}^{2}-\mu_{A l}(E) C_{2}^{2}\right) \\
\psi_{\text {thin }}(E)=\left[1+C_{3} \frac{E}{E_{\mathrm{e}}}+\left(\frac{E}{E_{\mathrm{e}}}\right)^{2}\right]\left[\ln \left(\frac{E_{e}\left(E_{\mathrm{e}}-E\right)}{E}+1.65\right)-0.5\right]\end{array}$ \\
\hline 13 & this study (with $511 \mathrm{keV}$ ) & - & 5 & in 12 above, replace $\psi_{\text {thin }}(E)$ with $\psi_{\text {thin }}(E)+C_{4} \frac{\delta\left(E-E_{511}\right)}{d E_{511}}$ \\
\hline
\end{tabular}


give the function some flexibility. Note that $\psi\left(E_{m}\right)=0$. In function $2, \psi\left(E_{m}\right)=0$,

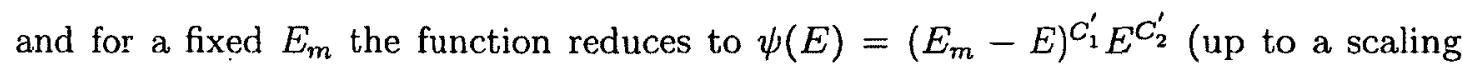
factor). Function 3 is an example of the class of thick-target formulae which use reasonable approximations to account for the spreading and slowing down of electrons in the target. This class of functions is not analyzed further because they: (a) are heavily tailored, which restricts how they can be parameterized, (b) are complex and not portable, (c) generally have free parameters to which the spectral fitting/unfolding is not very sensitive (e.g., $C_{2}$ in function 3 , which represents the atomic number of the flattening filter material), and, (d) require additional numerical integration which adds unnecessary noise during spectral unfolding. In function 4 , bremsstrahlung photons are assumed to be produced in the first thin-target layer, then attenuated by an effective exponential. The thin target spectrum is a parameterization of the Schiff spectrum in the forward direction, with zero photon emission angle. Function 5 uses the Schiff spectrum integrated over all photon emission angles [Eq. 3BS(e) in Koch and Motz, ${ }^{24}$ not reproduced for brevity], and attenuated by an energy-dependent exponential. The function has very large correlation arnong its free parameters in the exponent, and very strong nonlinearity with respect to $C_{2}$. In function 6 , the energy fluence is represented by a linear function of $E$ for $E \leq C_{1}$, where $C_{1}$ is the most probable energy, and by a Gaussian function with a spread of $C_{2}$ for $E \geq C_{1}$. The function is continuous at $E=C_{1}$, and depends very weakly on $E_{m}$ through $C_{2}$. In function 7 , the dependence on $E_{m}$ is implicit through the limits of the spectrum, and the sampling of this function can be done using the standard gamma distribution routines. Function 8 uses a trigonometric function to describe $\psi(E)$, with $\psi\left(E_{m}\right)=0$. Function 9 uses Kramers' spectrum attenuated by a flattening filter made of a known material, with its effective thickness as a free parameter. Function 10 was proposed as a better version of function 7 , with explicit dependence on $E_{m}$ and with $\psi\left(E_{m}\right) \doteq 0$; its last exponential represents attenuation in 
a flattening filter made of steel, with the effective thickness as a free parameter, $C_{3}$. In function 11 the first two factors with square brackets are the 'Fatigue Life' distribution (http://www.itl.nist.gov/div898/handbook/eda/section3/eda366a.htm), while the last factor is the Fermi density distribution. The authors used a four-parameter version of the function, in which $C_{4}$ and $C_{5}$ are expressed as fractions of $E_{m}$.

Each function in Table B.2 is fitted to each spectrum in the benchmark set of 65 spectra. The Levenberg-Marquardt least-squares algorithm ${ }^{70}$ is used to minimize $\% \Delta^{s}(\psi)$ (defined in Table B.1), which requires analytical first order derivatives with respect to each free parameter. For highly-nonlinear parameters in some functions, an exhaustive grid search is performed while minimizing $\% \Delta^{s}(\psi)$ with respect to all other parameters. Functions 2, 5 and 11 are the least robust, and function 8 is sensitive to the values of the lowest energy.

For a given spectrum, $s$, five $\% \Delta^{s}$ metrics (defined in Table B.1) are used to quantify the quality of fit of each function. Since $E_{m}$ is ill defined for typical clinical linacs, the fitted endpoint energy is compared to both the true $E_{e}\left[\right.$ through $\left.\% \Delta^{s}\left(E_{e}\right)\right]$ and the true $E_{m}$ [through $\left.\% \Delta^{s}\left(E_{m}\right)\right]$. For the functions with indefinite tails (functions 6, 7 and 11), an arbitrary cutoff ( $E$ at which $\psi \approx 3 \% \psi_{\text {peak }}$ ) is used to represent the fitted $E_{m}$. The five 'overall' $\% \Delta$ metrics facilitate quantitative and compact comparison of the functions against each other.

The robustness of each function is qualitatively investigated by using each function to unfold known spectra from analytical transmission data smeared with computational Gaussian noise (similar to the methods employed in §2.5).

The five overall $\% \Delta$ metrics for the quality of fits of different functions to the benchmark set are shown in Table B.3. The following observations can be made from the table. The large $\% \Delta$ values for the functions with less than four free parameters 
Table B.3: Quantitative evaluation of the fit quality of the functional forms listed in Table B.2 to the benchmark set of spectra described in App. A. Notation is defined in Table B.1. Parameterized thick-target formulae (function 3 and the like) are not analyzed. From Paper I.

\begin{tabular}{|c|c|c|c|c|c|c|c|c|}
\hline \multirow[b]{2}{*}{ Index } & \multirow[b]{2}{*}{ author(s) } & \multirow[b]{2}{*}{$n_{p}$} & \multirow{2}{*}{$\begin{array}{c}\text { Robust- } \\
\text { ness }\end{array}$} & \multicolumn{5}{|c|}{$\% \Delta(X), X$ is: } \\
\hline & & & & $\psi$ & $E_{e}$ & $E_{m}$ & $E_{m p}$ & $E_{a v}$ \\
\hline 1 & Dance and Baggerly ${ }^{97}$ & 2 & good & 27.7 & 3.9 & 6.8 & 69.3 & 38.4 \\
\hline 2 & Tarasko et al ${ }^{98}$ & 6 & poor & 15.2 & 20.8 & 16.3 & 45.4 & 17.4 \\
\hline 3 & Ahnesjö and Andreo ${ }^{20}$ & 4 & - & - & - & - & - & - \\
\hline 4 & Baker $;^{99}$ Baker et $a l^{65}$ & 4 & good & 2.5 & 1.9 & 4.4 & 9.1 & 1.2 \\
\hline 5 & Krmar et $a l^{71}$ & 4 & poor & 2.2 & 2.8 & 6.2 & 3.6 & 0.8 \\
\hline 6 & Bloch and McDonough ${ }^{67}$ & 2 & fair & 13.2 & 29.7 & 25.1 & 40.0 & 5.9 \\
\hline 7 & Fippel $^{100}$ & 3 & good & 18.6 & 43.9 & 38.8 & 32.1 & 7.3 \\
\hline 8 & Sawchuk 101 & 2 & fair & 27.0 & 19.6 & 15.3 & 62.0 & 10.7 \\
\hline 9 & Hinson and Bourland ${ }^{49}$ & 2 & good & 10.1 & 5.9 & 6.1 & 24.6 & 3.4 \\
\hline 10 & Sikora et $a l^{102}$ & 4 & fair & 6.6 & 6.5 & 5.0 & 12.2 & 5.2 \\
\hline 11 & Davidson et $a l^{103}$ & 5 & poor & 3.0 & 7.1 & 3.4 & 8.5 & 0.8 \\
\hline 12 & this study (no $511 \mathrm{keV}$ ) & 4 & good & 1.7 & 1.4 & 4.3 & 3.9 & 0.6 \\
\hline 13 & this study (with $511 \mathrm{keV}$ ) & 5 & - & 1.5 & 1.4 & 4.3 & 3.6 & 0.6 \\
\hline
\end{tabular}

(functions 1, 6, 7, 8 and 9) indicate that a truly accurate and flexible function requires at least four free parameters, one of which is the endpoint energy. The proposed function offers the lowest $\% \Delta$ values while still being robust. Functions 4,5 and 11 give the closest $\% \Delta$ values to the proposed function. However, functions 5 and 11 are not robust, and reducing the number of their free parameters to improve robustness has a significant effect on their accuracy and flexibility. Therefore it can be concluded that function 4 offers the second best overall performance (after the proposed function).

Examples of the fits of different functions to the benchmark set are shown in Fig. B.1 and they are discussed here panel-by-panel. In panel (a), the fits of function 1 are clearly different from the original spectra. Function 9 is similar in form to function 1 , but it performs better because the argument in its exponent is more representative of linac components. For function 8 , the quality of fit indicates that a trigonometric function does not provide a particularly good representation of linac spectra, and the fit quality 
gets worse for softer beams. In panel (b), function 2 is not robust and does not produce accurate fits despite having the largest number of free parameters. For function 6, its Gaussian nature at the higher-energy end leads to long unrealistic tails, and thus to large $\% \Delta\left(E_{m}\right)$ and $\% \Delta\left(E_{e}\right)$ values in Table B.3. Its straight line in the lower-energy part becomes problematic when $E_{m p} / E_{m}<0.1$ (i.e., softer beams), leading to a large $\% \Delta\left(E_{n n p}\right)$ value. Panel (c) shows fits of functions 7 and 10 together because the latter was introduced as a better version of the former. Function 7 has long high energy tails and thus large $\% \Delta\left(E_{m}\right)$ and $\% \Delta\left(E_{e}\right)$ values. The effect of the high energy cutoff in function 10 is obvious in the fit. Panel (d) shows that the fits of function 4 to central-axis spectra are almost identical to those of the proposed function, but the fits to off-axis spectra are worse. This is a direct result of the use of a forward-directed thin-target spectrum in function 4 as opposed to the use of the patterns common in thin-target spectra, integrated over all photon emission angles as done in the proposed form. Panel (e) shows that the fits of function 5 are comparable to those of the proposed function, except that function 5 is very non-robust. When its number of free parameters is reduced to improve robustness the fit quality deteriorates, as shown in panel (e). Panel (f) shows that function 11 matches well the higher end of the spectra that have large energy spread whereas the proposed function produces a hard cutoff at an effective $E_{e}$. However, function 11 produces the same tail whether or not the original spectrum has it, as shown for the NPL spectrum; function 11 is also not robust.

The effect of the differences between the original and the fitted spectra on depthdose curves is investigated using EGSnrc for the Varian $6 \mathrm{MV}$ beam with a flattening filter. For functions with $\% \Delta(\psi)$ values of only a few per cent, the depth of maximum dose, $d_{\text {mas }}$, remains within $0.5 \mathrm{~mm}$ of its true value, and the per cent depth dose at $10 \mathrm{~cm}, \% d d(10)$ change by a few tenths of a per cent. For functions with larger $\% \Delta(\psi)$ values, $d_{\text {max }}$ changes by up to $2 \mathrm{~mm}$ and $\% d d(10)$ by up to $3 \%$. 


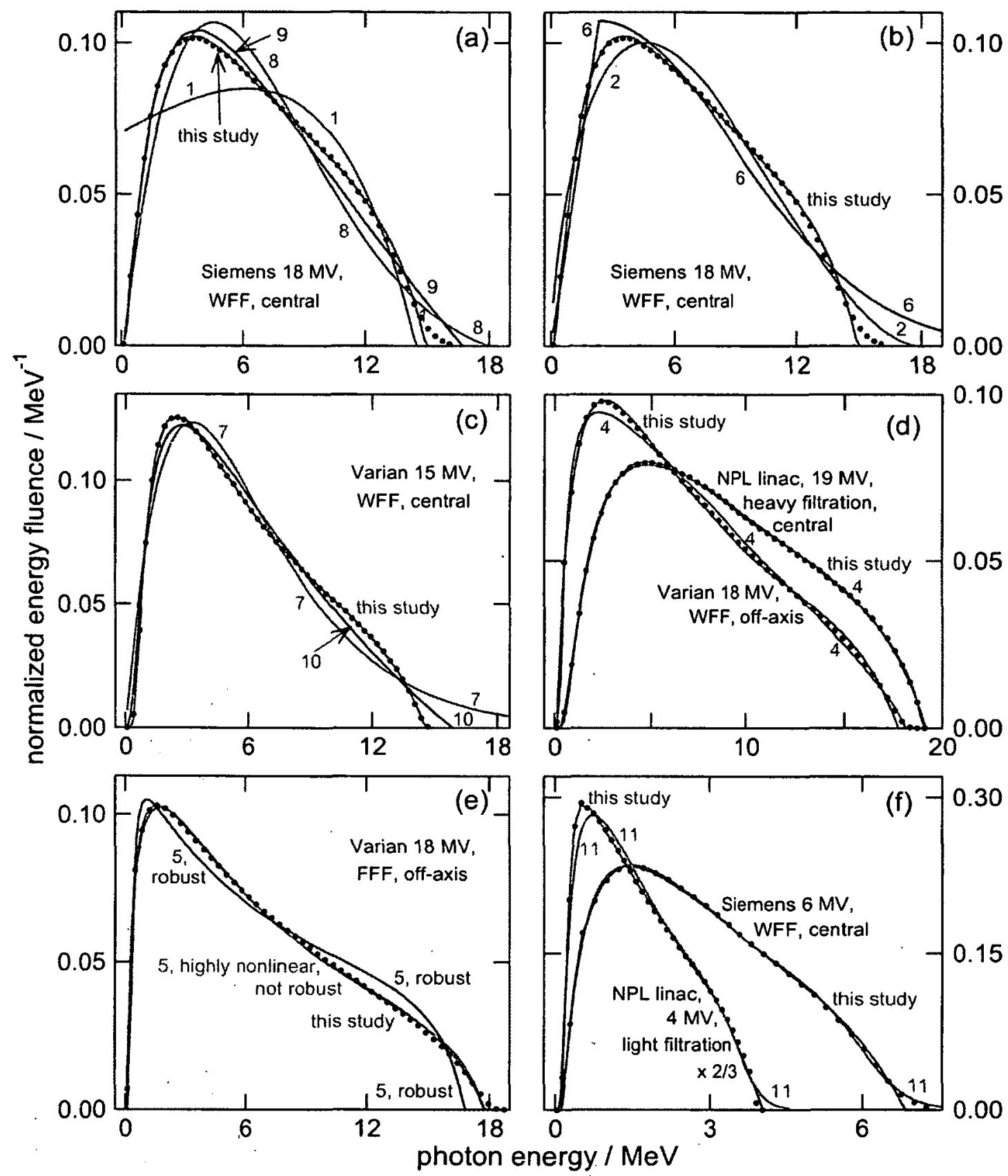

Figure B.1: Examples of the fits of different functions from the literature (solid lines) to Monte Carlo spectra (dots) from the benchmark set of 65 spectra (described in App. A). The fits are typical of others. The fits of the proposed function are also shown for comparison. The terms 'central', 'off-axis', 'WFF' and 'FFF' refer to, respectively, a central-axis spectrum, an off-axis spectrum, with flattening filter, and flattening-filter free. For graph clarity, only every other original Monte Carlo point is shown. Spectra are normalized to unit energy fluence. From Paper I. 


\section{Appendix C}

\section{Refining NIST XCOM photon}

\section{cross sections in EGSnrc}

This appendix addresses two issues related to the implementation of NIST XCOM photon cross sections in EGSnrc. The first is related to incoherent scattering and the second is related to pair/triplet cross sections. Paper V contains more details.

Incoherent scattering cross sections: EGSnrc has an option to use NIST XCOM data, but the option does not include using XCOM incoherent scattering data. Instead, the incoherent scattering cross sections are calculated internally in EGSnrc using the KleinNishina formula, modified (if requested) by binding effects and radiative corrections. Fig. C.1a shows comparisons between the internal EGSnrc incoherent scattering cross sections and the XCOM online data (queried at the exact energies of the EGSnrc grid). Differences of the order of $1 \%$ exist at low and high energies. The upgrade made in this study is that the XCOM incoherent scattering data are made available in EGSnrc. Data were queried from the online XCOM at 150 energies logarithmically spaced between $1 \mathrm{keV}$ and $100 \mathrm{GeV}$ for elements with $\mathrm{Z}=1-100$. XCOM data already include radiative corrections, therefore they are divided by the magnitude of the internal EGSnrc radiative correction to allow EGSnrc to keep its explicit modelling of radiative corrections, which can result in additional particles (e.g., in double Compton scattering). To use exactly $\mathrm{XCOM}$ in EGSnrc, the internal radiative corrections should be turned ON, and binding 


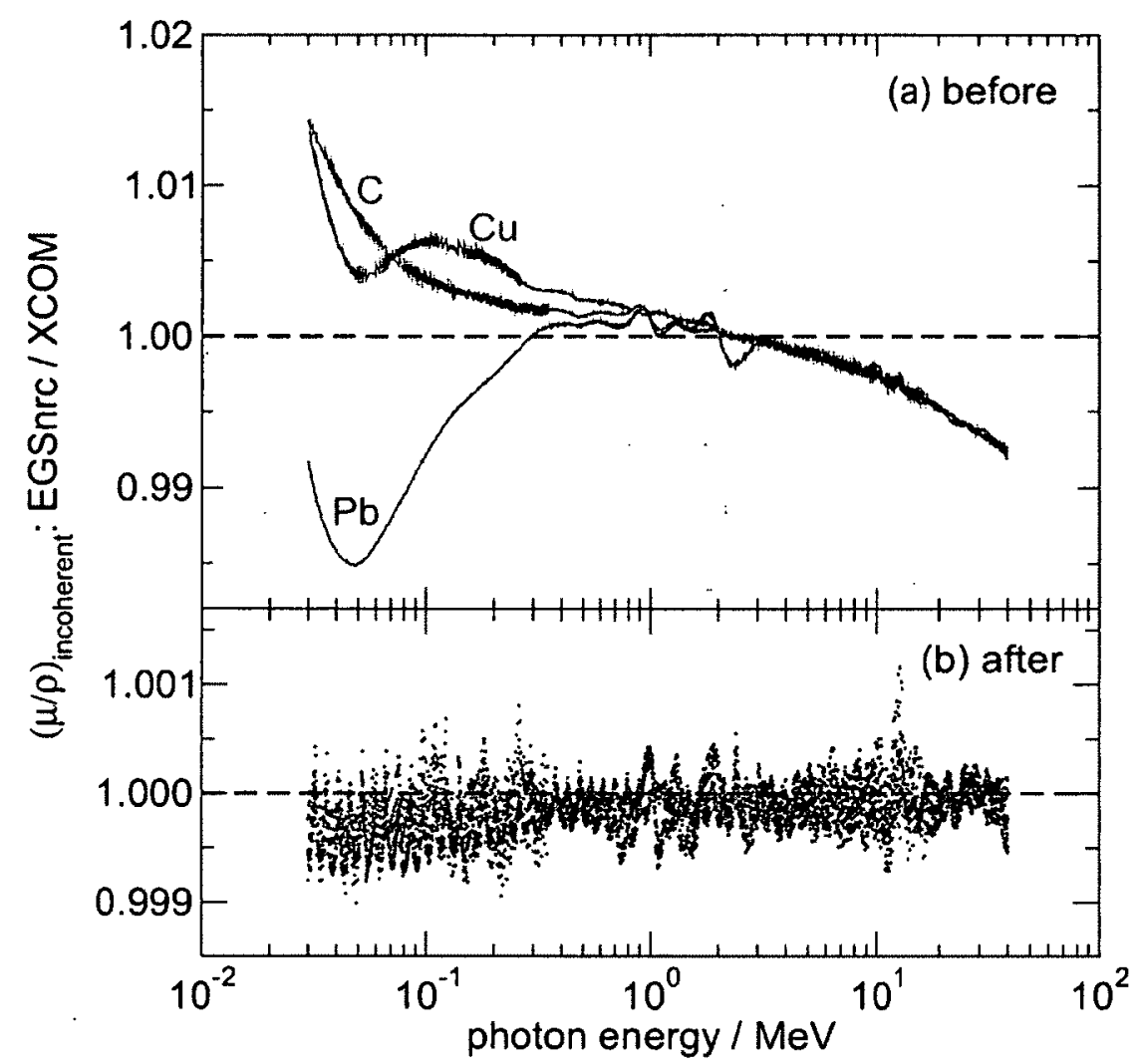

Figure C.1: Ratio of the incoherent scattering cross sections in EGSnrc to their respective NIST XCOM values. The EGSnrc cross sections are as follows. Panel (a): Data from internal EGSnrc calculations of incoherent scattering cross sections, including binding and radiative effects. Panel (b): New data using XCOM-based cross sections without disabling the internal EGSnrc feature of explicit modelling of radiative corrections. Note the different scales of the ordinates. From Paper V.

effects should be set to norej to disable on-the-fly corrections for them because XCOM data already include binding effects. Fig. C.1b shows that the new data used by EGSnrc agree with the online XCOM data wihin $0.05 \%$.

Pair/triplet cross sections: When XCOM is requested, EGSnrc reads at the start of a simulation the XCOM pair and triplet cross sections. However, pair and triplet cross sections at energies other than those of the input grid differ from the online XCOM data by up to $1.5 \%$ (Fig. C.2a). This is despite the fact that both EGSnrc and the online 


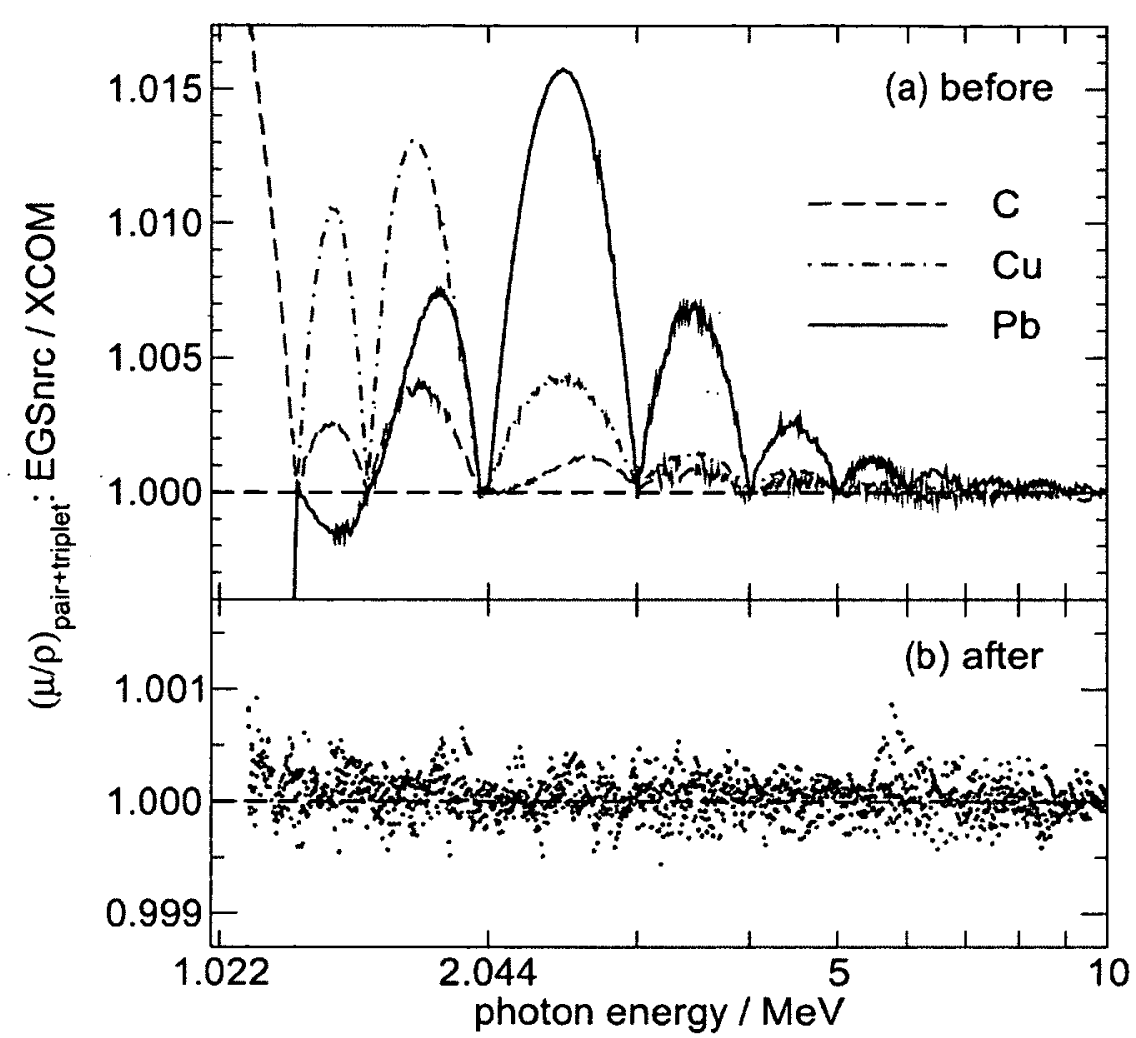

Figure C.2: Ratio of the pair + triplet cross sections in EGSnrc to their respective NIST XCOM values. The EGSnrc cross sections are as follows. Panel (a): Data based on XCOM values but with the original coarse energy grid. Panel (b): New XCOM-based data with a variable-resolution energy grid. Note the different scales of the ordinates. From Paper V.

$\mathrm{XCOM}$ use the same interpolation: linear in $\ln \left[\left(1-E_{t h} / E\right)^{3} \mu\right]$ versus $\ln (E)$, where $E$ is the photon energy, $\mu$ is the cross section and $E_{t h}$ is the production threshold (1.022 $\mathrm{MeV}$ for pair and $2.044 \mathrm{MeV}$ for triplet). The effect is due to the coarse fixed resolution of the energy grid for the input data to EGSnrc. The upgrade recreates the input pair/triplet data to EGSnrc from the online XCOM data using a variable-resolution energy grid that is denser in the energy regions where the effect is appreciable. Data are generated for elements with $\mathrm{Z}=1-100$. For pair, 150 energies are used between $1.03 \mathrm{MeV}$ and $100 \mathrm{GeV}$. For triplet, 115 energies are used between $2.05 \mathrm{MeV}$ and $100 \mathrm{GeV}$. The refined data generally agree with the online XCOM within $0.05 \%$ (Fig. C.2b). 


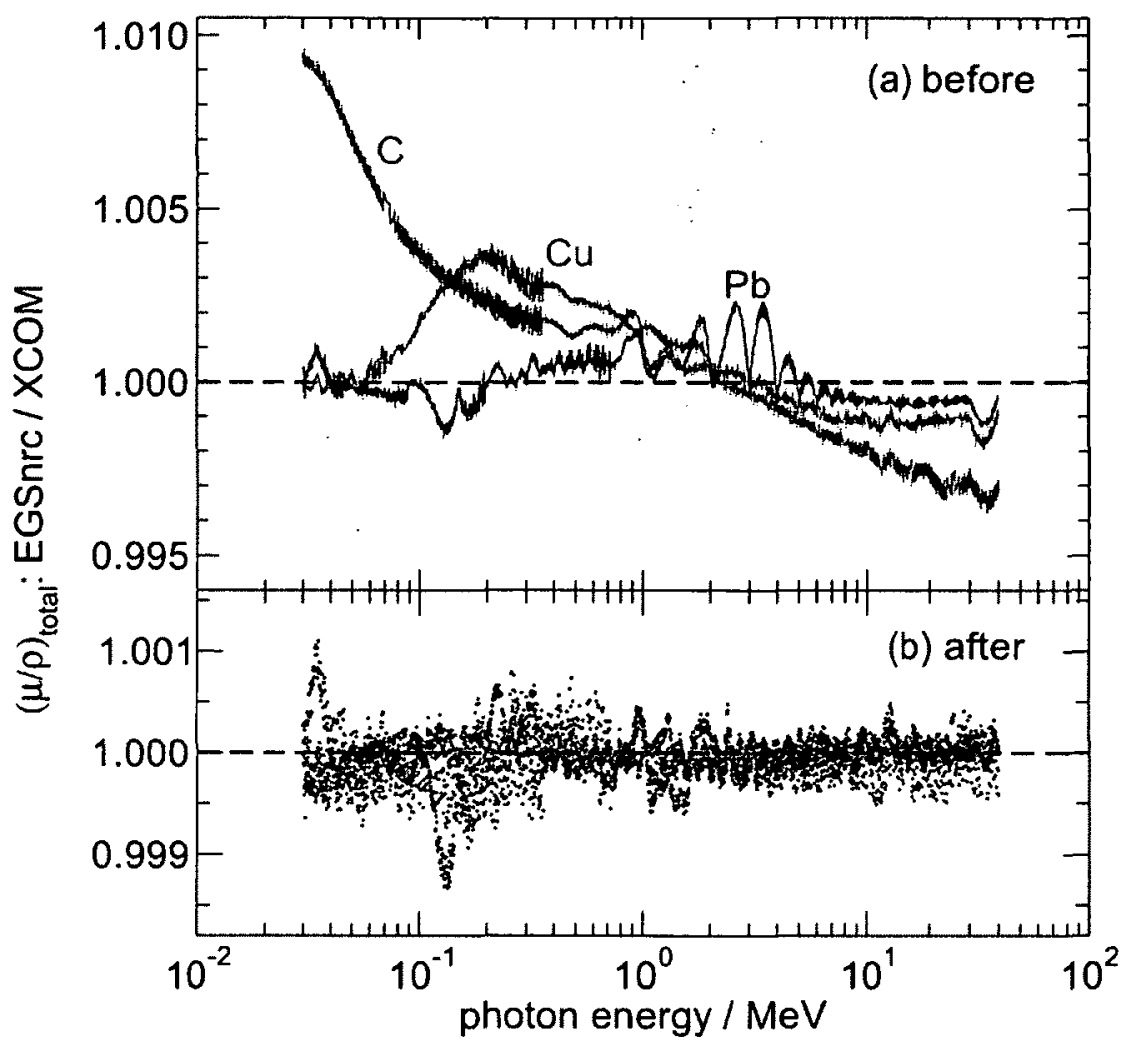

Figure C.3: Ratio of the total photon cross sections in EGSnrc (excluding the photonuclear cross sections of App. D) to their respective NIST XCOM values. The EGSnrc cross sections are as follows. Panel (a): Data based on Figs. C.1a and C.2a. Panel (b): New data from this study based on Figs. C.1b and C.2b. The components of the photoelectric effect and coherent scattering in the total cross section are not modified in either panel. Note the different scales of the ordinates. From Paper V.

Total cross sections: Comparisons of the total cross sections before and after the upgrade are shown in Fig. C.3. In Fig. C.3a, incoherent scattering differences lead to differences in the total cross section of up to $0.3 \%$ at higher energies. The pair/triplet issue causes differences up to $0.2 \%$ in the energy range $1-5 \mathrm{MeV}$ because its contribution to the total cross section is not large. To put these differences in context, for monoenergetic photons attenuated by two orders of magnitude, a $0.3 \%$ error in the cross section leads to a $1.4 \%$ error in the transmission data. The refined data generally agree with the online XCOM within $0.05 \%$. All EGSnrc calculations in this study use the refined data. 


\section{Appendix D}

\section{Implementing photonuclear}

\section{attenuation in EGSnrc}

This appendix provides a brief description of the upgrade made in this study to the EGSnrc system to model photonuclear attenuation. Report II contains the full implementation details.

Input photonuclear cross sections: Cross section data are generated using the comprehensive IAEA compilation of evaluated photonuclear data. ${ }^{36}$ Data for the constituent isotopes of natural elements $(Z=1-100)$ are individually extracted using the IAEA online interface (http://www-nds.iaea.org/exfor/endf.htm). The ENDF/B-VII. 1 library is used for most isotopes. When an isotope is missing from that library, other evaluated libraries (JENDL/PD-2004 and TENDL-2011) and individual

publications are used. Data are generated offline for the natural elements using the cross section data of their constituent isotopes, and their NIST isotopic abundances (http://www.nist.gov/pml/data/comp.cfm). Some of the generated data are visually compared against the graphical data available for some natural elements in the Atlas of Giant Dipole Resonances. ${ }^{104}$ Since the current version of the implementation in EGSnrc does not model secondary particles $(n, p, \alpha$, etc), only the total photonuclear cross sections are generated. The cross sections are prepared in the typical EGSnrc format of $[\ln$ (energy in $\mathrm{MeV}$ ), $\ln$ (cross section in barns/atom)]. For each natural 
element, the energy grid is 200 points, equi-spaced on a linear energy scale between the lowest threshold energy among all its constituent isotopes and the highest energy that has data available. These photonuclear cross sections are used as the default, but the user is given the option of user-supplied photonuclear data as well. Cross sections for any mixture or compound are generated internally in EGSnrc at runtime using the standard mixing rules.

Implementation: In the main EGSnrc input file, the user is given the option to turn the photonuclear effect ON or OFF for the full simulation geometry, or on a region-by-region basis. The region-by-region option is useful for, e.g., isolating the photonuclear effect from the bremsstrahlung target or the attenuators. When the photonuclear option is turned $\mathrm{ON}$ in a region, the photon mean free path in the medium of that region is shortened accordingly. After transporting the photon to the interaction site, an interaction type is sampled according to the relative cross section of each interaction. In the current version of the implementation, if the interaction is photonuclear, the photon is discarded without energy deposition and without generating secondary particles. This is sufficient for the purposes of this study. Infrastructure is created for future developers to implement secondary particle generation and transport.

Validation: BEAMnrc ${ }^{37,38} /$ cavity $^{39}$ are used to model the transmission measurement setup of this study for the $30 \mathrm{MV}$ beam from the NRC research linac and using $\mathrm{C}$ and $\mathrm{Pb}$ attenuators. Simulations are performed with photonuclear attenuation once turned ON and once OFF in the geometric region of the attenuator. EGSnrc results are compared to the calculations using Eq.1.1 (p. 8) for the same configuration. Fig. D.1 shows perfect agreement between the two methods, which validates the mechanics of the implementation in EGSnrc. Note that unlike the EGSnrc simulations, the calculations using Eq.1.1 do not include non-ideal attenuation effects such as forward scatter. However, for the ratio of the transmission data with and without photonuclear attenu- 


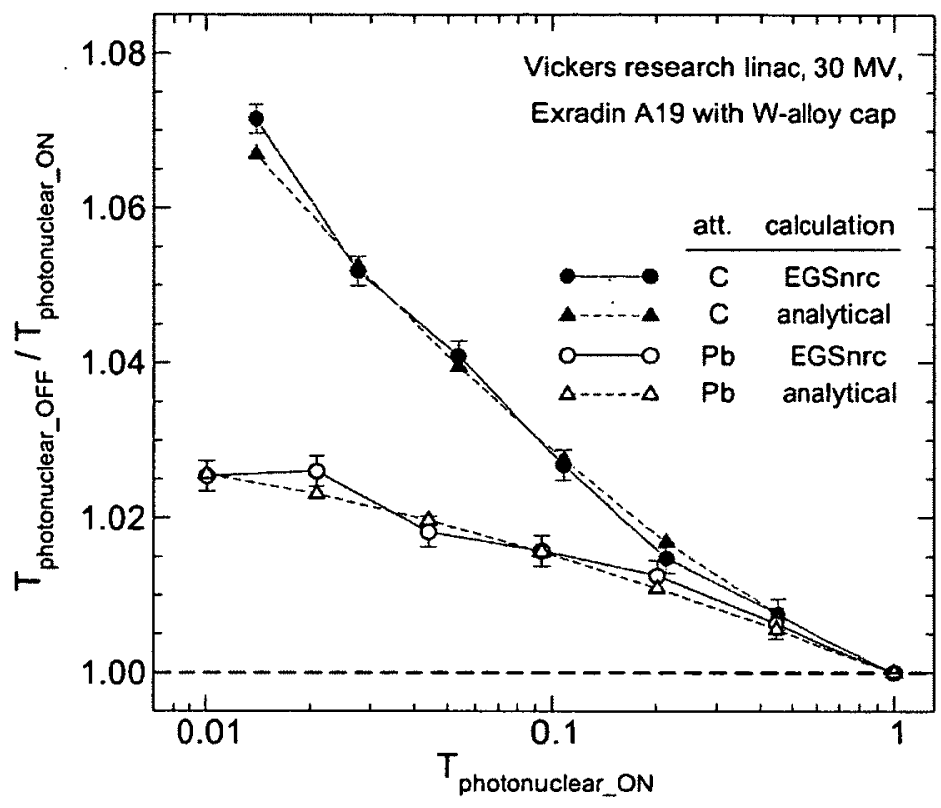

Figure D.1: To validate the implementation of photonuclear attenuation in EGSnrc, the figure shows EGSnrc results versus analytical calculations (Eq. 1.1 p. 8) for the effect of the photonuclear component on transmission, $T$. From Paper V.

ation, these non-ideal effects cancel out almost identically in the EGSnrc results, which makes the comparison in Fig. D.1 valid.

Finally, as seen in Fig. D.1, the $\sim 7 \%$ effect of photonuclear attenuation on the simulated transmission data justifies the efforts to implement it in EGSnrc. 


\section{References}

[1] B. L. Werner, I. J. Das, F. M. Khan, and A. S. Meigooni, Dose perturbations at interfaces in photon beams, Med. Phys. 14, 585 - 595 (1987).

[2] P. M. Charland, I. J. Chetty, L. D. Paniak, B. P. Bednarz, and B. A. Fraass, Enhanced spectral discrimination through the exploitation of interface effects in photon dose data, Med. Phys. 31, $264-276$ (2004).

[3] E. Tonkopi, M. McEwen, B. R. B. Walters, and I. Kawrakow, Influence of ion chamber response on in-air profile measurements in megavoltage photon beams, Med. Phys. 32, 2918 - 2927 (2005).

[4] P. R. Almond, P. J. Biggs, B. M. Coursey, W. F. Hanson, M. S. Huq, R. Nath, and D. W. O. Rogers, AAPM's TG-51 protocol for clinical reference dosimetry of high-energy photon and electron beams, Med. Phys. 26, 1847 - 1870 (1999).

[5] IAEA, Absorbed Dose Determination in External Beam Radiotherapy: An International Code of Practice for Dosimetry Based on Standards of Absorbed Dose to Water, volume 398 of Technical Report Series, IAEA, Vienna, 2001.

[6] B. A. Faddegon, M. Asai, J. Perl, C. Ross, J. Sempau, J. Tinslay, and F. Salvat, Benchmarking of Monte Carlo simulation of bremsstrahlung from thick targets at radiotherapy energies, Med. Phys. 35, $4308-4317$ (2008).

[7] B. Nordell and A. Brahme, Angular distribution and yield from bremsstrahlung targets, Phys. Med. Biol. 29, $797-810$ (1984).

[8] G. E. Desobry and A. L. Boyer, Bremsstrahlung review: An analysis of the Schiff spectrum, Med. Phys. 18, $497-505$ (1991).

[9] G. E. Desobry and A. L. Boyer, An analytic calculation of the energy fluence spectrum of a linear accelerator, Med. Phys. 21, $1943-1952$ (1994).

[10] H. I. Amols and D. L. Zellmer, Microdosimetry of $10-15 \mathrm{MeV}$ bremsstrahlung $x$ rays, Med. Phys. 11, $247-253$ (1984).

[11] B. A. Faddegon, C. K. Ross, and D. W. O. Rogers, Forward-directed bremsstrahlung of $10-$ to $30-\mathrm{MeV}$ electrons incident on thick targets of $\mathrm{Al}$ and $\mathrm{Pb}$, Med. Phys. 17, 773 - 785 (1990). 
[12] B. A. Faddegon, C. K. Ross, and D. W. O. Rogers, Angular distribution of bremsstrahlung from $15 \mathrm{MeV}$ electrons incident on thick targets of $\mathrm{Be}, \mathrm{Al}$ and $\mathrm{Pb}$, Med. Phys. 18, $727-739$ (1991).

[13] R. E. Bentley, J. C. Jones, and S. C. Lillicrap, X-ray spectra from accelerators in the range 2 to $6 \mathrm{MeV}$, Phys. Med. Biol. 12, $301-314$ (1967).

[14] L. B. Levy, R. G. Waggener, W. D. McDavid, and W. H. Payne, Experimental and calculated bremsstrahlung spectra from a $25-\mathrm{MeV}$ linear accelerator and a 19- $\mathrm{MeV}$ betatron, Med. Phys. 1, $62-67$ (1974).

[15] D. J. Landry and D. W. Anderson, Measurement of accelerator bremsstrahlung spectra with a high-efficiency Ge detector, Med. Phys. 18, 527 - 532 (1991).

[16] H. W. Koch and R. E. Carter, Determination of the energy distribution of bremsstrahlung from 19.5-MeV electrons, Phys. Rev. 77, 165-174 (1950).

[17] R. M. Warner Jr and E. F. Shrader, Angle-energy distribution of radiation from high-energy electron accelerators, Rev. Sci. Instrum. 25, 663 - 667 (1954).

[18] N. K. Sherman, K. H. Lokan, R. M. Hutcheon, L. W. Funk, W. R. Brown, and $\mathrm{P}$. Brown, Bremsstrahlung radiators and beam filters for $25-\mathrm{MeV}$ cancer therapy, Med. Phys. 1, 185 - 192 (1974).

[19] R. Nath and R. J. Schulz, Determination of high-energy x-ray spectra by photoactivation, Med. Phys. 3, 133 - 141 (1976).

[20] A. Ahnesjö and P. Andreo, Determination of effective bremsstrahlung spectra and electron contamination for photon dose calculations, Phys. Med. Biol. 34, 1451 - 1464 (1989).

[21] O. Sauer and M. Neumann, Reconstruction of high-energy bremsstrahlung spectra by numerical analysis of depth-dose data, Radiother. Oncol. 18, $39-47$ (1990).

[22] L. D. Paniak and P. M. Charland, Enhanced bremsstrahlung spectrum reconstruction from depth dose gradients, Phys. Med. Biol. 50, $3245-3261$ (2005).

[23] E. S. M. Ali, M. R. McEwen, and D. W. O. Rogers, On using depth-dose measurements to unfold linac photon spectra, Med. Phys., to be submitted (2012).

[24] H. W. Koch and J. W. Motz, Bremsstrahlung cross-section formulas and related data, Rev. Mod. Phys. 31, 920 - 955 (1959).

[25] L. I. Schiff, Energy-angle distribution of thin target bremsstrahlung, Phys. Rev. $83,252-253$ (1951).

[26] S. M. Seltzer and M. J. Berger, Bremsstrahlung spectra from electron interactions with screened atomic nuclei and orbital electrons, Nucl. Inst. Meth. B 12, $95-$ 134 (1985). 
[27] S. M. Seltzer and M. J. Berger, Bremsstrahlung energy spectra from electrons with kinetic energy from $1 \mathrm{keV}$ to $10 \mathrm{GeV}$ incident on screened nuclei and orbital electrons of neutral atoms with $\mathrm{Z}=1-100$, Atomic Data and Nuclear Data Tables 35, $345-418$ (1986).

[28] H. K. Tseng and R. H. Pratt, Exact screened calculations of atomic-field bremsstrahlung, Phys. Rev. A3, $100-115$ (1971).

[29] E. G. A. Aird and F. T. Farmer, The design of a thimble chamber for the Farmer dosemeter, Phys. Med. Biol. 17, 169 - 174 (1972).

[30] I. Kawrakow, Accurate condensed history Monte Carlo simulation of electron transport. I. EGSnrc, the new EGS4 version, Med. Phys. 27, $485-498$ (2000).

[31] I. Kawrakow, E. Mainegra-Hing, D. W. O. Rogers, F. Tessier, and B. R. B. Walters, The EGSnrc Code System: Monte Carlo simulation of electron and photon transport, NRC Technical Report PIRS-701 v4-2-3-2, National Research Council Canada, Ottawa, Canada. http://www.irs.inms.nrc.ca/inms/irs/EGSnrc/EGSnrc.html, 2011.

[32] I. Kawrakow, Accurate condensed history Monte Carlo simulation of electron transport. II. Application to ion chamber response simulations, Med. Phys. 27, $499-513(2000)$.

[33] I. Kawrakow, Electron impact ionization cross sections for EGSnrc, Med. Phys. (abstract) 29, 1230 (2002).

[34] F. Tessier and I. Kawrakow, Calculation of the electron-electron bremsstrahlung cross-section in the field of atomic electrons, Nucl. Inst. Meth. B 266, $625-634$ (2008).

[35] M. J. Berger, J. H. Hubbell, S. M. Seltzer, J. Chang, J. S. Coursey, R. Sukumar, D. S. Zucker, and K. Olsen, XCOM: Photon cross section database (version 1.5), Technical report, NIST, Gaithersburg, MD, http://physics.nist.gov/xcom, 2010.

[36] IAEA, Handbook of photonuclear data for applications: cross sections and spectra, Technical Report TECDOC 1178, IAEA. Available at:

http://www-nds.iaea.org/photonuclear, 2000.

[37] D. W. O. Rogers, B. A. Faddegon, G. X. Ding, C.-M. Ma, J. Wei, and T. R. Mackie, BEAM: A Monte Carlo code to simulate radiotherapy treatment units, Med. Phys. 22, 503 - 524 (1995).

[38] D. W. O. Rogers, B. Walters, and I. Kawrakow, BEAMnrc Users Manual, NRC Technical Report PIRS-509(A) revL, National Research Council of Canada, Ottawa, Canada. http://www.irs.inms.nrc.ca/BEAM/beamhome.html, 2011.

[39] I. Kawrakow, E. Mainegra-Hing, F. Tessier, and B. R. B. Walters, The EGSnrc $\mathrm{C}++$ class library, Technical Report PIRS-898 (rev A), National Research Council of Canada, Ottawa, Canada. http://www.irs.inms.nrc.ca/EGSnrc/PIRS898/, 2009. 
[40] D. W. O. Rogers, I. Kawrakow, J. P. Seuntjens, B. R. B. Walters, and E. Mainegra-Hing, NRC user codes for EGSnrc, NRC Technical Report PIRS-702 (rev C), National Research Council of Canada, Ottawa, Canada. http://www.irs.inms.nrc.ca/EGSnrc/PIRS702/, 2011.

[41] I. Kawrakow, D. W. O. Rogers, and B. Walters, Large efficiency improvements in BEAMnrc using directional bremsstrahlung splitting, Med. Phys. 31, 2883 2898 (2004)

[42] L. Silberstein, Determination of the spectral composition of x-ray radiation from filtration data, J. Optical Society of America 22, 265 - 280 (1932).

[43] P.-H. Huang, K. R. Kase, and B. E. Bjärngard, Spectral characterization of 4 MV bremsstrahlung by attenuation analysis, Med. Phys. 8, 368 - 374 (1981).

[44] C. R. Baker and K. K. Peck, Reconstruction of $6 \mathrm{MV}$ photon spectra from measured transmission including maximum energy estimation, Phys. Med. Biol. 42, 2041 - 2051 (1997).

[45] P. Francois, F. Coste, J. Bonnet, and O. Caselles, Validation of reconstructed bremsstrahlung spectra between $6 \mathrm{MV}$ and $25 \mathrm{MV}$ from measured transmission data, Med. Phys. 24, $769-773$ (1997).

[46] T. Shimozato, K. Tabushi, S. Kitoh, Y. Shiota, C. Hirayama, and S. Suzuki, Calculation of $10 \mathrm{MV}$ x-ray spectra emitted by a medical linear accelerator using the BFGS quasi-Newton method, Phys. Med. Biol. 52, 515 - 523 (2007).

[47] W. H. Hinson, W. T. Kearns, A. F. deGuzman, and J. D. Bourland, Photon spectral characteristics of dissimilar $6 \mathrm{MV}$ linear accelerators, Med. Phys. 35, $1698-1702$ (2008).

[48] A. Nisbet, H. Weatherburn, J. D. Fenwick, and G. McVey, Spectral reconstruction of clinical megavoltage photon beams and the implications of spectral determination on the dosimetry of such beams, Phys. Med. Biol. 43, 1507 - 1521 (1998).

[49] W. H. Hinson and J. D. Bourland, Spectral reconstruction of high energy photon beams for kernel based dose calculations, Med. Phys. 29, 1789 - 1796 (2002).

[50] A. Piermattei, G. Arcovito, L. Azario, C. Bacci, L. Bianciardi, E. De Sapio, and C. Giacco, A study of quality of bremsstrahlung spectra reconstructed from transmission measurements, Med. Phys. 17, 227 - 233 (1990).

[51] A. Catala, P. Francois, J. Bonnet, and C. Scouamec, Reconstruction of $12 \mathrm{MV}$ bremsstrahlung spectra from measured transmission data by direct resolution of the numeric system AF $=$ T, Med. Phys. 22, $3-10$ (1995).

[52] R. M. Harrison, G. D. Lambert, and C. L. Chapple, Spectral estimation and contrast calculation in the design of contrast-detail test objects for radiotherapy portal imaging, Phys. Med. Biol. 38, 545 - 556 (1993). 
[53] P.-H. Huang, K. R. Kase, and B. E. Bjärngard, Reconstruction of 4-MV bremsstrahlung spectra from measured transmission data, Med. Phys. 10, $778-$ 785 (1983).

[54] R. G. Waggener, M. M. Blough, J. A. Terry, D. Chen, N. E. Lee, S. Zhang, and W. D. McDavid, X-ray spectra estimation using attenuation measurements from 25 kVp to 18 MV, Med. Phys. 26, 1269 - 1278 (1999).

[55] M. Partridge, Reconstruction of megavoltage photon spectra from electronic portal imager derived transmission measurements, Phys. Med. Biol. 45, N115 - N131 (2000).

[56] P.-H. Huang, K. R. Kase, and B. E. Bjärngard, Simulation studies of 4-MV x-ray spectral reconstruction by numerical analysis of transmission data, Med. Phys. $9,695-702(1982)$.

[57] D. Sheikh-Bagheri and D. W. O. Rogers, Sensitivity of megavoltage photon beam Monte Carlo simulations to electron beam and other parameters, Med. Phys. 29, $379-390$ (2002).

[58] B. R. Archer and L. K. Wagner, A Laplace transform pair model for spectral reconstruction, Med. Phys. 9, $844-847$ (1982).

[59] B. R. Archer, P. R. Almond, and L. K. Wagner, Application of a Laplace transform pair model for high energy $x$-ray spectral reconstruction, Med. Phys. 12, $630-$ 633 (1985).

[60] S. D. Ahuja, P. G. Steward, T. S. Roy, and E. D. Slessinger, Estimated spectrum of a 4-MV therapeutic beam, Med. Phys. 13, $368-373$ (1986).

[61] P. Francois, A. Catala, and C. Scouarnec, Simulation of x-ray spectral reconstruction from transmission data by direct resolution of the numeric system AF $=\mathrm{T}$, Med. Phys. 20, $1695-1703$ (1993).

[62] S. Hussain, Artificial neural network model for spectral construction of a linear accelerator megavoltage photon beam, UKSim/AMSS IEEE 1st Intl Conf. on Intelligent Systems, Modelling and Simulation, Liverpool, England , 86-91 (2010).

[63] B. Armbruster, R. J. Hamilton, and A. K. Kuehl, Spectrum reconstruction from dose measurements as a linear inverse problem, Phys. Med. Biol. 49, 5087-5099 (2004).

[64] M. Manciu, F. Manciu, T. Vulcan, E. Nes, and R. Waggener, Robust megavoltage $\mathrm{X}$-ray spectra estimation from transmission measurements, J. X-Ray Sci. Technol. $17,85-99$ (2009).

[65] C. R. Baker, B. Ama'ee, and N. M. Spyrou, Reconstruction of megavoltage photon spectra by attenuation analysis, Phys. Med. Biol. 40, $529-542$ (1995). 
[66] M. Krmar, J. Slivka, I. Bikit, M. Vesković, and L. Conkić, Evaluation of bremsstrahlung spectra generated by a $4-\mathrm{MeV}$ linear accelerator, Med. Phys. 23, $651-654$ (1996).

[67] P. Bloch and J. McDonough, Extraction of the photon spectra from measured beam parameters, Med. Phys. 25, $752-757$ (1998).

[68] M. S. MacPherson and C. K. Ross, A magnetic spectrometer for electron energy calibration, Technical Report PIRS-0617 (59 pp), IRS, NRC, Ottawa, Canada. http://irs.inms.nrc.ca/publications/reports/pdf/PIRS-0617-1998.pdf, 1998.

[69] C. K. Ross, M. R. McEwen, A. F. McDonald, C. D. Cojocaru, and B. A. Faddegon, Measurement of multiple scattering of 13 and $20 \mathrm{MeV}$ electrons by thin foils, Med. Phys. 35, 4121 - 4131 (2008).

[70] D. W. Marquardt, An algorithm for least-squares estimation of nonlinear parameters, SIAM J. Appl. Math. 11, $431-441$ (1963).

[71] M. Krmar, J. Slivka, I. Bikit, M. Vesković, L. Čonkić, M. Bistrović, and A. Rudić, A new method for the measurement of bremsstrahlung spectra, Phys. Med. Biol. $38,533-544$ (1993).

[72] A. Iwasaki, M. Kubota, J. Hirota, A. Fujimori, K. Suzaki, M. Aoki, and Y. Abe, Characteristic features of a high-energy x-ray spectra estimation method based on the Waggener iterative perturbation principle, Med. Phys. 33, $4056-4063$ (2006).

[73] B. R. Muir and D. W. O. Rogers, Monte Carlo calculations of $k_{Q}$, the beam quality conversion factor, Med. Phys. 37, 5939 - 5950 (2010).

[74] H. E. Johns and J. R. Cunningham, The physics of radiology, 4th ed., Charles C. Thomas, Springfield, Illinois, 1983.

[75] F. McCarthy, V. Sahajwalla, J. Hart, and N. Saha-Chaudhury, Influence of ash on interfacial reactions between coke and liquid iron, Metall. Mater. Trans. B 34B, $573-580$ (2003).

[76] A. Piermattei, G. Arcovito, F. A. Bassi, and L. Azario, Reconstruction of 9 MV bremsstrahlung spectrum by numerical analysis of measured transmission data, Physica Medica 1, 43 - 57 (1987).

[77] J. P. McCaffrey, B. Downton, H. Shen, D. Niven, and M. McEwen, Pre-irradiation effects on ionization chambers used in radiation therapy. Phys. Med. Biol. 50, N121 - N133 (2005).

[78] M. R. McEwen, Measurement of ionization chamber absorbed dose $k_{Q}$ factors in megavoltage photon beams, Med. Phys. 37, $2179-2193$ (2010).

[79] R. E. Bentley, Uncertainty in Measurement: The ISO Guide, 6th ed, National Measurement Laboratory CSIRO, Australia, 2003. 
[80] N. Takata and Y. Morishita, Effect of radiation-induced charge accumulation on build-up cap on the signal current from an ionisation chamber, Radiat. Prot. Dosim. 145, 21 - 27 (2011).

[81] B. R. Muir, M. R. McEwen, and D. W. O. Rogers, Beam quality conversion factors for parallel-plate ionization chambers in MV photon beams, Med. Phys. $39,1618-1631$ (2012).

[82] J. Borg, I. Kawrakow, D. W. O. Rogers, and J. P. Seuntjens, Experimental verification of EGSnrc Monte Carlo calculated ion chamber response in low energy photon beams, in Proc. 22nd Ann. Int. Conf. of IEEE Engineering in Medicine and Biology Society, pages 3152-3155, Vol, 4, IEEE, 2000.

[83] J. P. Seuntjens, I. Kawrakow, J. Borg, F. Hobeila, and D. W. O. Rogers, Calculated and measured air-kerma response of ionization chambers in low and medium energy photon beams, in Recent developments in accurate radiation dosimetry, Proc. of an Int'l Workshop, edited by J. P. Seuntjens and P. Mobit, pages $69-$ 84, Medical Physics Publishing, Madison WI, 2002.

[84] B. R. Muir, M. R. McEwen, and D. W. O. Rogers, Measured and Monte Carlo calculated $k_{Q}$ factors: accuracy and comparison, Med. Phys. 38, $4600-4609$ (2011).

[85] J. H. Hubbell, Review of photon interaction cross section data in the medical and biological context, Phys. Med. Biol. 44, R1 - R22 (1999).

[86] D. W. O. Rogers, Analytic and graphical methods for assigning errors to parameters in non-linear least squares fitting, Nucl Inst and Meth 127, $253-260$ (1975).

[87] F. Tessier and I. Kawrakow, Effective point of measurement of thimble ion chambers in megavoltage photon beams, Med. Phys. 37, $96-107$ (2010).

[88] J. Wulff, J. T. Heverhagen, K. Zink, and I. Kawrakow, Investigation of systematic uncertainties in Monte Carlo-calculated beam quality correction factors, Phys. Med. Biol. 55, 4481 - 4493 (2010).

[89] P. Andreo, D. T. Burns, and F. Salvat, On the uncertainties of photon mass energy-absorption coefficients and their ratios for radiation dosimetry, Phys. Med. Biol. 57, 2117 - 2136 (2012).

[90] B. R. B. Walters, D. W. O. Rogers, and K. R. Shortt, Monte Carlo simulation of a cesium irradiator using BEAM, Med. Phys. 24, (abstract) 981 (1997).

[91] ICRU, Stopping powers for electrons and positrons, ICRU Report 37, ICRU, Bethesda, MD., 1984.

[92] B. R. B. Walters and D. W. O. Rogers, Monte Carlo estimates of \%dd(10) $)_{x}$ for the NPL photon beams, Report PIRS 659, NRC. Available at: http://www.physics.carleton.ca/ drogers/pubs/papers/WR00.pdf, 2000.

REFERENCES 
[93] R. Jeraj, T. R. Mackie, J. Balog, G. Olivera, D. Pearson, J. Kapatoes, K. Ruchala, and P. Reckwerdt, Radiation characteristics of helical tomotherapy, Med. Phys. 31, $396-404$ (2004).

[94] J. F. Briesmeister, MCNP - A general Monte Carlo N-particle transport code, version 4c3, Los Alamos National Laboratory Report LA-12625 (Los Alamos, NM) (1999).

[95] F. Araki, Monte Carlo study of a Cyberknife stereotactic radiosurgery system, Med. Phys. 33, $2955-2963$ (2006).

[96] H. A. Kramers, On the theory of x-ray absorption and of the continuous x-ray spectrum, Phil. Mag. 46, 836 - 871 (1923).

[97] W. E. Dance and L. L. Baggerly, Investigations of electron interactions with matter, Part 1: Bremsstrahlung production in aluminum and iron, Report CR334, NASA, 1965.

[98] M. Z. Tarasko, A. S. Soldatov, and V. E. Rudnikov, Description of bremsstrahlung spectra from a thick target for 4-12 MeV electrons, Atomnaya Énergiya (Soviet Atomic Energy) 65, $290-291$ (1988).

[99] C. R. Baker, Reconstruction of clinical bremsstrahlung spectra in the range 4 to $30 \mathrm{MeV}$, Ph.D. thesis, University of Surrey, U.K., 1993.

[100] M. Fippel, Fast Monte Carlo dose calculation for photon beams based on the VMC electron algorithm, Med. Phys. 26, 1466 - 1475 (1999).

[101] S. Sawchuk, Monte Carlo estimate to improve photon energy spectrum reconstruction, in Advanced Monte Carlo for Radiation Physics, Particle Transport Simulation and Applications, Proc. Monte Carlo 2000 Conf., Lisbon, edited by A. Kling, F. Barão, M. Nakagawa, L. Távora, and P. Vaz, pages $383-388$, Springer, Berlin, 2001.

[102] M. Sikora, O. Dohm, and M. Alber, A virtual photon source model of an Elekta linear accelerator with integrated mini MLC for Monte Carlo based IMRT dose calculation, Phys. Med. Biol. 52, 4449 - 4463 (2007).

[103] S. Davidson, J. Cui, D. Followill, G. Ibbott, and J. Deasy, A flexible Monte Carlo tool for patient or phantom specific calculations: comparison with preliminary validation measurements, J. Phys. Conf. Series 102, 012004 (6 pp) (2008).

[104] A. Varlamov, V. Varlamov, D. Rudenko, and M. Stepanov, Atlas of giant dipole resonances: parameters and graphs of photonuclear reaction cross sections, Report INDC(NDS)-394, IAEA Nuclear Data Section, IAEA, Vienna, 1999. 